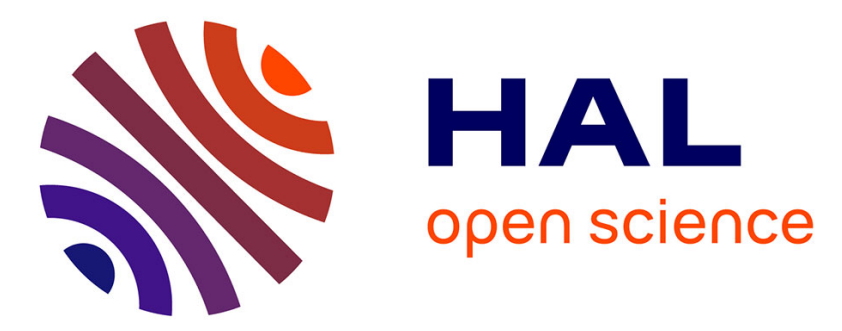

\title{
Stabilization of parabolic nonlinear systems with finite dimensional feedback or dynamical controllers. Application to the Navier-Stokes system
}

\author{
Mehdi Badra, Takéo Takahashi
}

\section{- To cite this version:}

Mehdi Badra, Takéo Takahashi. Stabilization of parabolic nonlinear systems with finite dimensional feedback or dynamical controllers. Application to the Navier-Stokes system. SIAM Journal on Control and Optimization, 2011, 49 (2), pp.420-463. 10.1137/090778146 . hal-00431041v2

\section{HAL Id: hal-00431041 \\ https://hal.science/hal-00431041v2}

Submitted on 8 Nov 2010

HAL is a multi-disciplinary open access archive for the deposit and dissemination of scientific research documents, whether they are published or not. The documents may come from teaching and research institutions in France or abroad, or from public or private research centers.
L'archive ouverte pluridisciplinaire HAL, est destinée au dépôt et à la diffusion de documents scientifiques de niveau recherche, publiés ou non, émanant des établissements d'enseignement et de recherche français ou étrangers, des laboratoires publics ou privés. 


\title{
Stabilization of parabolic nonlinear systems with finite dimensional feedback or dynamical controllers. Application to the Navier-Stokes system
}

\author{
Mehdi Badra*, Takéo Takahashi ${ }^{\dagger}$
}

November 4, 2010

\begin{abstract}
Let $A: \mathcal{D}(A) \rightarrow \mathcal{X}$ be the generator of an analytic semigroup and $B: \mathcal{V} \rightarrow\left[\mathcal{D}\left(A^{*}\right)\right]^{\prime}$ a relatively bounded control operator. In this paper, we consider the stabilization of the system $y^{\prime}=A y+B u$ where $u$ is the linear combination of a family $\left(v_{1}, \ldots, v_{K}\right)$. Our main result shows that if $\left(A^{*}, B^{*}\right)$ satisfies a unique continuation property and if $K$ is greater or equal to the maximum of the geometric multiplicities of the unstable modes of $A$, then the system is generically stabilizable with respect to the family $\left(v_{1}, \ldots, v_{K}\right)$. With the same functional framework, we also prove the stabilizability of a class of nonlinear systems when using feedback or dynamical controllers. We apply these results to stabilize the Navier-Stokes equations in 2D and in 3D by using boundary controls.
\end{abstract}

Key words. Parabolic equation, Navier-Stokes equations, feedback stabilization, Dirichlet boundary control, finite dimensional control, Lyapunov functional, Riccati equation.

AMS subject classifications. 93D15, 35B40, 35Q30, 76D05, 76D07, 76D55, 93B52, 93C20.

\section{Introduction}

The present paper provides a general framework for stabilization of non linear parabolic equation by means of finite dimensional feedback or dynamical control. One of the main motivations is the stabilization of the unstationary Navier-Stokes system around a stationary state. More precisely, let $\Omega$ be a bounded domain of $\mathbb{R}^{d}$, for $d=2$ or $d=3$, with a boundary $\partial \Omega$ of class $C^{2,1}$, and let $\left(w_{S}, q_{S}\right) \in\left(H^{2}(\Omega)\right)^{d} \times H^{1}(\Omega)$ be a solution of the stationary Navier-Stokes equations:

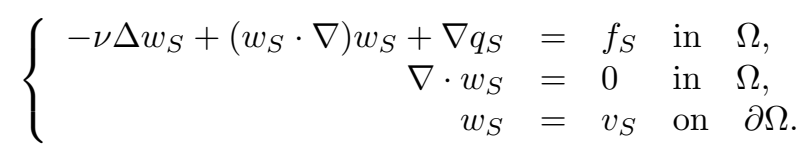

In this setting, $\nu>0$ is the viscosity coefficient. It is well-known that such a pair $\left(w_{S}, q_{S}\right)$ exists if $f_{S} \in\left(L^{2}(\Omega)\right)^{d}$ and $v_{S} \in\left(H^{\frac{3}{2}}(\partial \Omega)\right)^{d}$ satisfies $\int_{\Gamma} v_{S} \cdot n d \Gamma=0$ for each connected component $\Gamma$ of $\partial \Omega$, see for instance $[22$, Thm. VIII.4.1 and VIII.5.2, p.48]. Here $n$ denotes the unit normal vector to $\partial \Omega$ exterior to $\Omega$. Let us also consider a trajectory $\left(w_{U}, q_{U}\right)$ solution of the unstationary Navier-Stokes equations:

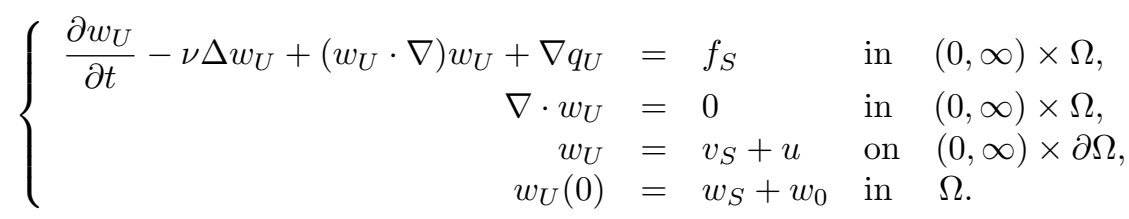

\footnotetext{
*Laboratoire LMA, UMR CNRS 5142, Université de Pau et des Pays de l'Adour, 64013 Pau Cedex, France (mehdi.badra@univ-pau.fr).

†Institut Élie Cartan, UMR 7502, INRIA, Nancy-Université, CNRS, BP239, 54506 Vandœuvre-lès-Nancy Cedex, France

$\ddagger$ Team-Project CORIDA. INRIA Nancy - Grand Est, 615, rue du Jardin Botanique. 54600 Villers-lès-Nancy. France (takahash@inria.fr).
} 
In the above system, the function $u$ at the boundary is a control used to "reach", at least asymptotically, the stationary solution $\left(w_{S}, q_{S}\right)$. More precisely, if we denote by $(w, q)$ the difference between the solution of $(1)$ and the stationary solution (2), it satisfies

$$
\left\{\begin{array}{rlll}
\frac{\partial w}{\partial t}-\nu \Delta w+\left(w_{S} \cdot \nabla\right) w+(w \cdot \nabla) w_{S}+(w \cdot \nabla) w+\nabla q & =0 & \text { in } \quad(0, \infty) \times \Omega, \\
\nabla \cdot w & =0 & \text { in } \quad(0, \infty) \times \Omega, \\
w & =u & \text { on } \quad(0, \infty) \times \partial \Omega \\
w(0) & = & w_{0} & \text { in } \quad \Omega,
\end{array}\right.
$$

and, for a prescribed rate of decrease $\sigma>0$, we want to find a control $u$ of finite dimension:

$$
u(t, x)=\sum_{j=1}^{K} u_{j}(t) v_{j}(x), \quad \bar{u}=\left(u_{1}, \ldots, u_{K}\right) \in L^{2}\left(\mathbb{R}^{K}\right)
$$

such that, for some norm $\|\cdot\|$ and at least for $w_{0}$ in a neighborhood of 0 , the following exponential decay holds:

$$
\|w(t)\|=\left\|w_{U}(t)-w_{S}\right\| \leq C e^{-\sigma t}\left\|w_{0}\right\|
$$

In (4), the number $K$ and the functions $v_{j}, j=1, \ldots K$, are fixed and will be determined later on, and $\bar{u}$ is a control function that we want to express with a feedback formulation. More precisely, we are interested in this paper in the stabilizability problem. We recall that the controllability problem would consist in finding a control allowing to reach in finite time a particular state (such as $w_{S}$ ). Our aim here is to obtain a closed-loop system, which means that at each time the control is a function of the state, in order to "reach" the state $w_{S}$ in infinite time. For more details on controllability and stabilizability questions, we refer for instance the reader to $[36,9,39,16]$.

In this article, we solve the above problem by using a classical strategy: we consider a feedback control $u(t)=\mathfrak{F}(w(t))$ where $\mathfrak{F}:\left(L^{2}(\Omega)\right)^{d} \rightarrow\left(L^{2}(\partial \Omega)\right)^{d}$ is obtained through the solution of a Riccati equation. This allows to stabilize the system (3) for $d=2$. However as explained in [33] or in [2], for $d=3$, the regularity needed for the nonlinear term imposes some compatibility condition on the feedback control $u$ which are not possible with this classical strategy. More precisely, for an initial datum $w_{0}$ which does not satisfy $\left.w_{0}\right|_{\partial \Omega}=\mathfrak{F}\left(w_{0}\right)$ it is not possible to define solution with a fixed-point technique.

One way to handle this difficulty is to consider "dynamical controllers": we take $u$ as the solution of an other equation which is controlled. For instance, if we assume that $u$ is of finite dimension as in (4), one can consider that the coefficients $\bar{u}$ are solutions of a linear ODE:

$$
\bar{u}^{\prime}=D_{K} \bar{u}+G_{K} \bar{g}, \quad \bar{u}(0)=\bar{u}_{0},
$$

where $D_{K}: \mathbb{R}^{K} \rightarrow \mathbb{R}^{K}$ and $G_{K}: \mathbb{R}^{K} \rightarrow \mathbb{R}^{K}$ are linear operators. In that case $\bar{g}: \mathbb{R} \rightarrow \mathbb{R}^{K}$ is the control for both $\bar{u}$ and $w$ and the feedback law is using both variables to stabilize the system. Notice that to have the continuity of the trace, and then to be able to define a 3-D fixed-point solution, we must impose an initial trace compatibility conditions $u(0)=\left.w_{0}\right|_{\partial \Omega}$, or equivalently:

$$
\sum_{j=1}^{K} u_{j}(0) v_{j}=w_{0} \quad \text { on } \quad \partial \Omega
$$

Here, to obtain a stabilization theorem for the 3-D Navier-Stokes system, we assume that $\left.w_{0}\right|_{\partial \Omega}=0$ and thus we consider (5) with the following initial condition:

$$
\bar{u}_{0}=0 \text {. }
$$

Notice that in [33], in the case of infinite dimensional feedback control, the author overcomes this difficulty of compatibility conditions by introducing a time dependent feedback law.

As a consequence of these differences between $d=2$ and $d=3$, in what follows our results have two forms: the case of "classical" finite-dimensional controllers (where $\bar{u}$ is directly given by a feedback law) and the case of finite-dimensional "dynamical" controllers (where $\bar{u}$ is solution of (5)).

Moreover, our approach is to consider a general functional framework so that our result could be applied to other systems: let $A: \mathcal{D}(A) \subset \mathcal{X} \rightarrow \mathcal{X}$ be a closed linear operator with compact resolvent in a real Hilbert 
space $\mathcal{X}$ and assume it is the infinitesimal generator of an analytic semigroup in $\mathcal{X}$. The last hypothesis implies in particular that there are a finite number of "unstable" modes: for any prescribed $\sigma>0$, there are only $N$ eigenvalues of $A$ with real part strictly greater than $-\sigma: \lambda_{k}, k=1, \ldots, N(N$ depending on $\sigma)$. Let us also consider a control operator $B: \mathcal{V} \rightarrow\left[\mathcal{D}\left(A^{*}\right)\right]^{\prime}$ (with $\mathcal{V}$ a real Hilbert space) which is strictly relatively bounded: for some $0 \leq \gamma<1$ and for $\lambda_{0}>0$ large enough, $\left(\lambda_{0}-A\right)^{-\gamma} B: \mathcal{V} \rightarrow \mathcal{X}$ is bounded. Finally, let us take a nonlinearity $F$ which can be "unbounded" (see (92) and (95)). Then our aim is to stabilize an infinite-dimensional system of the form

$$
y^{\prime}+A y+B u=F(y, u), \quad y(0)=y_{0} .
$$

With an additional property on $A$ (see (54)), one can prove the following results (see Theorem 15 and 18 ):

Theorem 1. Assume

1. Any $\varepsilon \in \mathcal{D}\left(A^{*}\right)$ which obeys $A^{*} \varepsilon=\bar{\lambda} \varepsilon$ and $B^{*} \varepsilon=0$ for some $\lambda \in\left\{\lambda_{k} \mid k=1, \ldots, N\right\}$ satisfies $\varepsilon=0$.

2. $K$ is greater or equal to the maximum of the geometric multiplicities of $\lambda_{k}, k=1, \ldots, N$ :

$$
K \geq \max \left\{\ell_{k} \mid k=1, \ldots, N\right\} .
$$

Then, there exists a family $v_{1}, \ldots, v_{K}$ of $\mathcal{V}$ and there exists a positive constant $c$ such that the following results hold:

- Assume ||$y_{0}|| \mid<c$ and assume $u=-\sum_{j=1}^{K}\left(v_{j} \mid B^{*} \Pi P_{N} y\right) \mathcal{v} v_{j}$, where $P_{N}$ is the projection on the unstable modes and where $\Pi$ is solution of a Riccati equation of finite-dimension (see (36)-(37)). Then the solution of (7) satisfies

$$
\left\|\left|y ( t ) \left\|\left|\leq\left\|\left|y_{0}\right|\right\| e^{-\sigma t}\right.\right.\right.\right.
$$

- Assume ||$\left|y_{0}, \bar{u}_{0}\right|||<c$, assume $\bar{g}=-G_{K}^{*} \Pi_{2} y_{N}-G_{K}^{*} \Pi_{3} \bar{u}$, where $\Pi_{2}$ and $\Pi_{3}$ are components of the solution $\Pi$ of a Riccati equation of finite-dimension (see (49)-(50)), and assume $G_{K}$ is invertible. Then the solution of (7), (5) satisfies

$$
\left\|\left|y(t), \bar{u}(t)\left\|\left|\leq\left\|y_{0}, \bar{u}_{0}\right\|\right| e^{-\sigma t} .\right.\right.\right.
$$

In the above result, the norm $\||\cdot|\|$ is defined precisely in Theorems 15 and 18 . Let us underline that the minimal number $K$ of controllers for which the above stabilization theorem is valid if $K=\max \left\{\ell_{k}\right\}$. In fact, the choice of the family $v_{1}, \ldots, v_{K}$ is not unique and more precisely, we prove that the suitable families form an open and dense subset of $\mathcal{V}^{K}$. This theorem could be applied to other parabolic systems than the Navier-Stokes equations, as soon as the two above conditions are satisfied. The second condition means that we have to take enough controllers and the first condition corresponds to a unique continuation property. This is the main point which has to be checked when considering different systems or different control operators. Let us remark that this condition is satisfied if the system $y^{\prime}=A y+B u$ is approximately controllable (see Theorem 6).

For instance, it is possible to apply the above general result to Boussinesq equations by using [25]. It is also important to remark the "linear" version of the above result $(F \equiv 0$ and $c=+\infty)$ could be also applied to improve previous result on the heat equation. This linear version could also be applied to some fluid-structure systems such as the motion of a rigid body in a viscous incompressible fluid; indeed, a linear problem associated to the nonlinear system is parabolic (see, for instance, [37]), however the fixed point strategy is quite different from the general theory presented here. Finally, let us notice that we use in an essential way the analyticity of the semigroup generated by $A$ and the fact that there is only a finite number of unstable eigenvalues and eigenvectors. The present framework does not apply for hyperbolic systems because there could be an infinite number of "unstable" modes. Even in the case of boundary layers model described by a degenerate parabolic equation as in $[12,11]$, because of the lack of analyticity of the semigroup and of the lack of compactness of the resolvent, our arguments fail. See $[14,13]$ for a complete study of the stabilization problem for the Crocco equation.

If we apply the above theorem to system (3), we deduce the following result (see Theorems 33 and 36 for precise statements):

Theorem 2. Assume $K$ is greater or equal to the maximum of the geometric multiplicities of the unstable modes of the Oseen operator associated to $w_{S}$ (see $\left.(97),(115)\right)$. There exists $\left(v_{1}, \ldots, v_{K}\right) \in\left(\mathbf{L}^{2}(\partial \Omega)\right)^{K}$ and there exists a positive constant $c$ such that the following results hold: 
- Assume $d=2, s \in\left[0, \frac{1}{2}\right)$ and $w_{0} \in \mathbf{H}^{s}(\Omega)$ such that $\nabla \cdot w_{0}=0$, assume $\left\|P w_{0}\right\|_{\mathbf{H}^{s}(\Omega)}<c$ and assume

$$
u_{j} \stackrel{\text { def }}{=}\left(\int_{\partial \Omega} v_{j} \cdot\left(B^{*} \Pi\right) P_{N} P w d \Gamma\right),
$$

where $P$ is the Leray projector and $\Pi$ is solution of a Riccati equation of finite-dimension (see Subsection 5.1 for the notations), then the solution of (3), (4) satisfies

$$
\|w(t)\|_{\mathbf{H}^{s}(\Omega)} \leq C\left\|P w_{0}\right\|_{\mathbf{H}^{s}(\Omega)} e^{-\sigma t} \quad \text { for all } t \geq 0 .
$$

- Assume $d=3, s \in\left[\frac{1}{2}, 1\right]$ and $w_{0} \in \mathbf{H}_{0}^{s}(\Omega)$ such that $\nabla \cdot w_{0}=0, \bar{u}_{0}=0$, assume $\left\|w_{0}\right\|_{\mathbf{H}_{0}^{s}(\Omega)}<c$ assume $G_{K}$ is invertible and assume

$$
\bar{g} \stackrel{\text { def }}{=}-G_{K}^{*} \Pi_{2} P_{N} P w-G_{K}^{*} \Pi_{3} \bar{u},
$$

where $\Pi_{2}$ and $\Pi_{3}$ are components of the solution $\Pi$ of a Riccati equation of finite-dimension. Then the solution of (3), (5) satisfies

$$
\|w(t)\|_{\mathbf{H}^{s}(\Omega)}+\|\bar{u}(t)\|_{\mathbb{R}^{K}} \leq C\left\|w_{0}\right\|_{\mathbf{H}^{s}(\Omega)} e^{-\sigma t} \quad \text { for all } t \geq 0 .
$$

In the above result we take a feedback control localized on the whole boundary. We have a similar result for internal control and for control localized on a part of the domain or of the boundary (see Section 5 for precise statements).

The works in the literature which are most relevant to our present paper are $[5,6,8,7,35]$. Indeed, the stabilization of the Navier-Stokes system by means of finite-dimensional feedback controllers is obtained in [8], in the case of an internal control, and in $[5,6,7,35]$, in the case of a boundary control and only for $d=2$, by using a Riccati strategy. The first (and main) step of the Riccati approach consists in proving the stabilizability of the Oseen system (which is obtained from (3) by linearizing around zero) by means of a finite dimensional control. It allows in a second step to define an auxiliary optimal control problem which provides a feedback controller stabilizing the Oseen system. Such a feedback law is obtained from the solution of an algebraic Riccati equation. Thus, in a third step, it remains to verify that the feedback controller also stabilizes the Navier-Stokes system. The stabilizability of the Oseen system obtained in $[5,6,7,8]$ is deduced from the exact controllability of the finite dimensional projected system related to the unstable eigenvalues of the Oseen operator, and the feedback law is obtained from the solution of an infinite dimensional Riccati equation. We underline that in $[5,6,7]$ it is assumed that the unstable spectrum is semi-simple, which is to say that algebraic and geometric multiplicity of each unstable eigenvalue coincide, or equivalently, that the finite dimensional projected operator is diagonalizable. Such an assumption is referred as the finite-dimensional spectral assumption (FDSA) and the authors postulate that the FDSA is also necessary for exact controllability of the projected system, see [6, Rem. 3.6.2 and Rem. 3.6.4]. In [35] the exact controllability of the finite dimensional unstable projected system is avoided, and the stabilizability by means of a finite dimensional control is simply obtained from the stabilizability by means of an infinite dimensional control which is proved in [32]. Moreover, the feedback control obtained in [35] is obtained from the solution of a finite dimensional Riccati equation stated in $\mathbb{R}^{N_{u} \times N_{u}}$ where $N_{u}$ is the dimension of the unstable space of the Oseen operator. In the present paper, we obtain the stabilizability of the Oseen system by proving the exact controllability of the projected system without assuming the FDSA, and we obtain a feedback control from a finite dimensional Riccati equation similarly as in [35].

However, in the above quoted work the question of finding the minimal number $K$ in (4) for which (3),(4) is stabilizable is not really clear. If $m_{a}$ denotes the greatest algebraic multiplicity of the unstable eigenvalues then the stabilization result obtained in [8] requires $K=m_{a}$ or $K=2 m_{a}$ actuators, according to the fact that the eigenvalues of maximum multiplicity is real or not, see [8, Rem. 3.9]. The proof relies on the linear independence of the family $\left(B^{*} \varepsilon_{k}\right)$, where $\left(\varepsilon_{k}\right)$ is a family of generalized eigenfunctions of $A^{*}$ related to the unstable eigenvalues and $B^{*}$ is the adjoint of the control operator, which in the present case is the restriction operator to the open subset where the control is acting, see [8, Claim. 3.3]. In [5, 6, 7], because the linear independence of the family $\left(B^{*} \varepsilon_{k}\right)$ corresponding to the boundary control is not known, the method of [8] does not apply and the number $K$ is simply the dimension of the unstable space of the Oseen operator. In fact, the linear independence of the family $\left(B^{*} \varepsilon_{k}\right)$ is known to be false in some simple situations. Consider for instance the Dirichlet Laplacian in $(0, \pi)^{d}$ with eigenvalues and eigenvectors given by

$$
\lambda_{\alpha}=\sum_{1 \leq j \leq d} \alpha_{j}^{2}, \quad \alpha=\left(\alpha_{1}, \ldots, \alpha_{d}\right) \in \mathbb{N}^{* d},
$$


and

$$
\varepsilon_{\alpha}(x)=\left(\frac{2}{\pi}\right)^{\frac{d}{2}} \prod_{1 \leq j \leq d} \sin \left(\alpha_{j} x_{j}\right) \quad x=\left(x_{1}, \ldots, x_{d}\right) \in(0, \pi)^{d} .
$$

In the case of Dirichlet control localized on $\Gamma_{0}=\left\{x \in(0, \pi)^{d} \mid x_{1}=0\right\}$, the function $B^{*} \varepsilon_{\alpha}$ is simply the normal derivative of $\varepsilon_{\alpha}$ on $\Gamma_{0}$ and has the following expression:

$$
-\frac{d \varepsilon_{\alpha}}{d x_{1}}\left(x^{\prime}\right)=-\alpha_{1}\left(\frac{2}{\pi}\right)^{\frac{d}{2}} \prod_{2 \leq j \leq d} \sin \left(\alpha_{j} x_{j}\right), \quad x^{\prime}=\left(0, x_{2}, \ldots, x_{d}\right) \in \Gamma_{0} .
$$

Then it is easily seen that for two eigenvalues $\lambda_{\alpha}$ and $\lambda_{\beta}$ where $\alpha=\left(\alpha_{1}, \ldots, \alpha_{d}\right)$ and $\beta=\left(\beta_{1}, \ldots, \beta_{d}\right)$ are such that $\alpha_{j}=\beta_{j}, j=2, \ldots, d$, we have $\beta_{1} \frac{d \varepsilon_{\alpha}}{d x_{1}}=\alpha_{1} \frac{d \varepsilon_{\beta}}{d x_{1}}$. Notice that in [35] the number $K$ is the number of linearly independent elements of $\left(B^{*} \varepsilon_{k}\right)$, which in view of the above example can be lower that the dimension of the unstable space, but which is not known in practice.

Some months after the submission of the present paper, we were informed of [4] where a general scheme for stabilization of linear evolution equations applicable to Navier-Stokes equations is described. Finite dimensional stabilizing feedback are obtained for a semi-simple unstable spectrum or for a linearly independent family $\left(B^{*} \varepsilon_{k}\right)$. In particular, under the last assumption, the author proposes a stabilization procedure referred as a "direct proportional stabilization of unstable modes" which is conceptually different from the Riccati approach and which does not rely on the resolution of an infinite dimensional Riccati equation. However, the number of actuators $K$ remains the same as in [8].

In the present paper we show that the minimal value of $K$ is the maximum of the geometric (and not algebraic) multiplicities of the unstable eigenvalues and that the linear independence of $\left(B^{*} \varepsilon_{k}\right)$ is not a necessary condition to construct a minimal and admissible family $\left(v_{1}, \ldots, v_{K}\right)$.

The rest of the paper is organized as follows. Section 2 is dedicated to notations and general definitions. Section 3 is devoted to general infinite dimensional parabolic systems: we characterize the families $\left(v_{1}, \ldots, v_{K}\right)$ for which the finite dimensional projected system on the unstable modes is exactly controllable, and then we apply a Riccati strategy to obtain a feedback or a dynamical control stabilizing the projected system. In Section 4 we apply the finite dimensional feedback or dynamical law obtained in Section 3 to the complete abstract evolution equation and we prove stabilization theorems with a Lyapunov approach. In Section 5 we apply the abstract stabilization results of Section 4 in the particular case of the Oseen and the Navier-Stokes systems.

\section{Notations and general functional framework}

Throughout what follows, the letter $C$ denotes a generic positive constant that may change from line to line. When particular positive constants are needed, we use a subscript: $C_{0}, C_{1}$, etc.

If $\mathcal{H}$ is a Hilbert space, we denote by $\|\cdot\|_{\mathcal{H}}$ its corresponding norm, we denote by $\mathcal{H}^{\prime}$ its dual space and by $\langle\cdot \mid \cdot\rangle_{\mathcal{H}^{\prime}, \mathcal{H}}$ the $\mathcal{H}^{\prime}-\mathcal{H}$ duality pairing. For two Hilbert spaces $\mathcal{H}_{1}$ and $\mathcal{H}_{2}$ we use the notation $\mathcal{H}_{1} \hookrightarrow \mathcal{H}_{2}$ to say that $\mathcal{H}_{1}$ is continuously embedded into $\mathcal{H}_{2}$, we denote by $\mathcal{L}\left(\mathcal{H}_{1}, \mathcal{H}_{2}\right)$ the space of all bounded linear operators from $\mathcal{H}_{1}$ into $\mathcal{H}_{2}$ and we use the shorter expression $\mathcal{L}(\mathcal{H})=\mathcal{L}(\mathcal{H}, \mathcal{H})$. For $0<T \leq \infty$, the space $L^{2}(0, T ; \mathcal{H})$ is the usual vector-valued Lebesgue space, $H^{s}(0, T ; \mathcal{H})$ for $s \geq 0$ is the usual vector-valued Sobolev space, $H_{0}^{s}(0, T ; \mathcal{H})$ denotes the closure in $H^{s}(0, T ; \mathcal{H})$ of the space of infinitely differentiable and compactly supported functions of $t \in] 0, T$ [ with values in $\mathcal{H}$, and $H^{-s}\left(0, T ; \mathcal{H}^{\prime}\right)$ denotes the dual space of $H_{0}^{s}(0, T ; \mathcal{H})$. We also define

$$
W\left(0, T ; \mathcal{H}_{1}, \mathcal{H}_{2}\right) \stackrel{\text { def }}{=} L^{2}\left(0, T ; \mathcal{H}_{1}\right) \cap H^{1}\left(0, T ; \mathcal{H}_{2}\right),
$$

and when $T=+\infty$ we use the shorter expressions $L^{2}(\mathcal{H})=L^{2}(0,+\infty ; \mathcal{H}), H^{s}(\mathcal{H})=H^{s}(0,+\infty ; \mathcal{H})$, and $W\left(\mathcal{H}_{1}, \mathcal{H}_{2}\right)=W\left(0,+\infty ; \mathcal{H}_{1}, \mathcal{H}_{2}\right)$. Moreover, $L^{\infty}(\mathcal{H})$ (resp. $\left.C_{b}(\mathcal{H})\right)$ is the space of bounded (resp. continuous and bounded) functions of $t \in\left[0, \infty\left[\right.\right.$ with values in $\mathcal{H}$. For $\theta \in(0,1)$ the space $\left[\mathcal{H}_{1}, \mathcal{H}_{2}\right]_{\theta}$ is the interpolation space obtained from $\mathcal{H}_{1}$ and $\mathcal{H}_{2}$ with the complex interpolation method [38, p.55]. We recall that if $\mathcal{H}_{1} \hookrightarrow \mathcal{H}_{2}$, then $W\left(\mathcal{H}_{1}, \mathcal{H}_{2}\right)$ is continuously embedded in $C_{b}\left(\left[\mathcal{H}_{1}, \mathcal{H}_{2}\right]_{1 / 2}\right)[38,1.8(2)$, p.44 and Rem.3 p.143]. Finally, we denote by $L_{\text {loc }}^{2}(\mathcal{H})$ the space of functions belonging to $L^{2}(0, T ; \mathcal{H})$ for all $T>0$, and we define $L_{\text {loc }}^{\infty}(\mathcal{H}), H_{\text {loc }}^{s}(\mathcal{H})$ etc, analogously.

If $L$ is a closed linear mapping on $\mathcal{H}$, we denote its domain by $\mathcal{D}(L)$, and $L^{*}$ denotes the adjoint of $L$. We recall that if $-L$ is the infinitesimal generator of an analytic semigroup on $\mathcal{H}$ of negative type [9, Part. II, 
Chap 1, Par. 2.2. p.91 and Par. 2.7. p.108] then $L^{-1} \in \mathcal{L}(\mathcal{H})$ [9, Part. II, Chap 1, Prop. 2.9. p.120] and the fractional powers $L^{\theta}$ for $\theta \in(0,1)$ are well-defined [9, Part. II, Chap 1, Par. 5. p.167]. Moreover, if we also suppose that the interpolation equality $[\mathcal{D}(L), \mathcal{H}]_{\frac{1}{2}}=\mathcal{D}\left(L^{\frac{1}{2}}\right)$ is true then the mapping $z \mapsto\left(z^{\prime}+L z, z(0)\right)$ is an isomorphism from $W(\mathcal{D}(L), \mathcal{H})$ onto $L^{2}(\mathcal{H}) \times \mathcal{D}\left(L^{\frac{1}{2}}\right)$ [9, Part. II, Chap 1, Thm. 3.1. p.143 and Par. 6 eq. (6.4)]. More generally, for $r \geq 0$ one set $\mathcal{H}^{r}=\mathcal{D}\left(L^{r}\right)$ and $\mathcal{H}^{-r}=\left[\mathcal{D}\left(L^{* r}\right)\right]^{\prime}$ and from a change of variable $y=L^{-r} z$ one easily obtain that the mapping

$$
y \mapsto\left(y^{\prime}+L y, y(0)\right): W\left(\mathcal{H}^{r+1 / 2}, \mathcal{H}^{r-1 / 2}\right) \rightarrow L^{2}\left(\mathcal{H}^{r-1 / 2}\right) \times \mathcal{H}^{r} \quad \text { is an isomorphism for all } r \in \mathbb{R} .
$$

In the present paper we consider the system

$$
y^{\prime}=A y+B u \in\left[\mathcal{D}\left(A^{*}\right)\right]^{\prime}
$$

where $A: \mathcal{D}(A) \subset \mathcal{X} \rightarrow \mathcal{X}$ is a closed linear operator with compact resolvent on a real Hilbert space $\mathcal{X}$ and which is the infinitesimal generator of an analytic semigroup on $\mathcal{X}$, and $B$ is a bounded linear operator from a real Hilbert space of control $\mathcal{V}$ with values in $\left.\left[\mathcal{D}\left(A^{*}\right)\right]^{\prime}\right)$. Here, $\left[\mathcal{D}\left(A^{*}\right)\right]^{\prime}$ denotes the dual space of $\mathcal{D}\left(A^{*}\right)$ with respect to the pivot space $\mathcal{X}$, it corresponds to the space $X_{-1}$ in the terminology of [39]. Notice that it will also be convenient in the sequel to view $A$ and $B$ as linear operators (still denoted by $A$ and $B$ ) in the complexified spaces

$$
\mathcal{Z} \stackrel{\text { def }}{=} \mathcal{X}+\imath \mathcal{X} \quad \text { and } \quad \mathcal{U}=\mathcal{V}+\imath \mathcal{V}
$$

Since $A$ generates an analytic semigroup on $\mathcal{Z}$, from $(8)$ we have that for $y_{0} \in \mathcal{Z}$ and a control function $u \in L_{\text {loc }}^{2}(\mathcal{U})$ system $(9)$ admits a unique solution $y \in W_{l o c}\left(\mathcal{Z} ;\left[\mathcal{D}\left(A^{*}\right)\right]^{\prime}\right)$ such that $y(0)=y_{0}$. Moreover, since it is also assumed that $A$ has compact resolvent, its spectrum $\Sigma$ then consists entirely of eigenvalues which are isolated points of the complex plane $\mathbb{C}[29$, III. Thm 6.29] and which are located in sector of a left half-plane with an opening angle strictly lower than $\pi$. There is a complex sequence $\left(\lambda_{k}\right)_{k \in \mathbb{N}^{*}}$ such that $\Sigma=\left\{\lambda_{k} \mid k \in \mathbb{N}^{*}\right\}$ and for a given $\sigma>0$ there is only a finite number $N$ of eigenvalues with real part strictly greater than $-\sigma$. More precisely we can reorder $\left(\lambda_{k}\right)_{k \in \mathbb{N}^{*}}$ so that the sequence $\left(\Re \lambda_{k}\right)_{k \in \mathbb{N}^{*}}$ is non increasing and we suppose that $\sigma>0$ and $N \in \mathbb{N}^{*}$ are such that:

$$
\cdots \leq \Re \lambda_{N+1}<-\sigma<\Re \lambda_{N} \leq \cdots \leq \Re \lambda_{2} \leq \Re \lambda_{1}
$$

Finally, we set:

$$
\Sigma_{N} \stackrel{\text { def }}{=}\left\{\lambda_{k} \mid k=1, \ldots, N\right\}
$$

\section{Controllability and stabilization of the linear projected system}

\subsection{Complex projected systems}

In the present section we split (9) in two equations, one related to the "unstable" modes $\Sigma_{N}$ and the other to the "stable" modes $\Sigma \backslash \Sigma_{N}$. First, let us decompose the space $\mathcal{Z}$ as the direct sum of two invariant subspaces of $A$ related to $\Sigma_{N}$ and $\Sigma \backslash \Sigma_{N}$. Let $\Gamma_{N}$ be a positively-oriented curve enclosing $\Sigma_{N}$ but no other point of the spectrum of $A$, and let $P_{N}: \mathcal{Z} \rightarrow \mathcal{Z}$ be the projection operator defined by

$$
P_{N} \stackrel{\text { def }}{=}-\frac{1}{2 \pi \imath} \int_{\Gamma_{N}}(\xi-A)^{-1} d \xi .
$$

It is well known that $P_{N}$ provides the following decomposition of $\mathcal{Z}$ :

$$
\mathcal{Z}=\mathcal{Z}_{N} \oplus \mathcal{Z}_{N}^{-} \quad \text { where } \quad \mathcal{Z}_{N} \stackrel{\text { def }}{=} P_{N}(\mathcal{Z}) \quad \text { and } \quad \mathcal{Z}_{N}^{-} \stackrel{\text { def }}{=}\left(I-P_{N}\right)(\mathcal{Z}),
$$

that $\mathcal{Z}_{N}$ and $\mathcal{Z}_{N}^{-}$are invariant subspaces under $A$, and that the spectra of the restricted operators $\left.A\right|_{\mathcal{Z}_{N}}$ and $\left.A\right|_{\mathcal{Z}_{N}^{-}}$are $\Sigma_{N}$ and $\Sigma \backslash \Sigma_{N}$ respectively [29, III. Thm 6.17]. In what follows, we use the notations:

$$
\left.A_{N} \stackrel{\text { def }}{=} A\right|_{\mathcal{D}(A) \cap \mathcal{Z}_{N}}: \mathcal{D}(A) \cap \mathcal{Z}_{N} \rightarrow \mathcal{Z}_{N} \quad \text { and }\left.\quad A_{N}^{-} \stackrel{\text { def }}{=} A\right|_{\mathcal{D}(A) \cap \mathcal{Z}_{N}^{-}}: \mathcal{D}(A) \cap \mathcal{Z}_{N}^{-} \rightarrow \mathcal{Z}_{N}^{-}
$$


Moreover, since $\Gamma_{N}$ is a bounded closed curve, $P_{N}$ is a bounded operator from $\mathcal{Z}$ into $\mathcal{D}(A)$ and the continuous embedding $\mathcal{Z}_{N} \hookrightarrow \mathcal{D}(A)$ holds. As a consequence, $P_{N}$ is a compact projection operator and then its range $\mathcal{Z}_{N}$ is necessarily finite dimensional. Next, since the spectrum of $A^{*}: \mathcal{D}\left(A^{*}\right) \subset \mathcal{Z} \rightarrow \mathcal{Z}$ (the adjoint of $A$ ) is exactly composed of the complex conjugates of the eigenvalues of $A$ [29, III. Thm 6.22], then we have another decomposition of $\mathcal{Z}$ as a direct sum of two invariant subspaces of $A^{*}$ :

$$
\mathcal{Z}=\mathcal{Z}_{N}^{*} \oplus \mathcal{Z}_{N}^{*-}, \quad \mathcal{Z}_{N}^{*} \stackrel{\text { def }}{=} P_{N}^{*}(\mathcal{Z}) \quad \text { and } \quad \mathcal{Z}_{N}^{*-} \stackrel{\text { def }}{=}\left(I-P_{N}^{*}\right)(\mathcal{Z}), \quad \text { where } \quad P_{N}^{*} \stackrel{\text { def }}{=}-\frac{1}{2 \pi \imath} \int_{\bar{\Gamma}_{N}}\left(\xi-A^{*}\right)^{-1} d \xi
$$

In the above setting, $P_{N}^{*}: \mathcal{Z} \rightarrow \mathcal{Z}$ is a projection operator with finite dimensional range $\mathcal{Z}_{N}^{*}$ included in $\mathcal{D}\left(A^{*}\right)$, and since $P_{N}^{*}$ is the adjoint of $P_{N}$ we deduce that their ranges have the same dimension [29, III. Thm 4.13]:

$$
\operatorname{dim}\left(\mathcal{Z}_{N}\right)=\operatorname{dim}\left(\mathcal{Z}_{N}^{*}\right)
$$

Notice that we also have the following characterization of $\mathcal{Z}_{N}$ and $\mathcal{Z}_{N}^{*}$ in terms of generalized eigenspaces:

$$
\mathcal{Z}_{N}=\bigoplus_{k=1}^{N} \operatorname{Ker}\left(A-\lambda_{k}\right)^{m_{k}} \quad \text { and } \quad \mathcal{Z}_{N}^{*}=\bigoplus_{k=1}^{N} \operatorname{Ker}\left(A^{*}-\bar{\lambda}_{k}\right)^{m_{k}}
$$

where $m_{k}$ is the multiplicity of the pole $\lambda_{k}$ in the resolvent of $A$, see $[20,35]$ and reference therein. Moreover, if we denote by $\mathcal{Z}_{N}^{\perp}$ (resp. $\mathcal{Z}_{N}^{* \perp}$ ) the orthogonal space of $\mathcal{Z}_{N}$ (resp. $\mathcal{Z}_{N}^{*}$ ) in $\mathcal{Z}$ then we verify that:

$$
\mathcal{Z}_{N}^{\perp}=\mathcal{Z}_{N}^{*-} \quad \text { and } \quad \mathcal{Z}_{N}^{* \perp}=\mathcal{Z}_{N}^{-}
$$

Next, we turn back to the solution $y \in W_{\text {loc }}\left(\mathcal{Z} ;\left[\mathcal{D}\left(A^{*}\right)\right]^{\prime}\right)$ of $(9)$ where $B \in \mathcal{L}\left(\mathcal{U},\left[\mathcal{D}\left(A^{*}\right)\right]^{\prime}\right)$ and $u \in L_{\text {loc }}^{2}(\mathcal{U})$. If we write $y=y_{N}+y_{N}^{-}$where $y_{N} \stackrel{\text { def }}{=} P_{N} y$ and $y_{N}^{-} \stackrel{\text { def }}{=}\left(I-P_{N}\right) y$ then there is a corresponding splitting of $(9)$ in two equations satisfied by $y_{N}$ and $y_{N}^{-}$respectively. In order to apply $P_{N}$ and $I-P_{N}$ to (9) we first need to extend their definition. Since $P_{N}^{*}$ is bounded from $\mathcal{Z}$ into $\mathcal{D}\left(A^{*}\right)$, the projection operator $P_{N}$ can be uniquely extended as a bounded operator from $\left[\mathcal{D}\left(A^{*}\right)\right]^{\prime}$ into $\mathcal{Z}_{N}$ as follows:

$$
\left(P_{N} e \mid \varepsilon\right)_{\mathcal{Z}}=\left\langle e \mid P_{N}^{*} \varepsilon\right\rangle_{\left[\mathcal{D}\left(A^{*}\right)\right]^{\prime}, \mathcal{D}\left(A^{*}\right)} \quad \forall(e, \varepsilon) \in\left[\mathcal{D}\left(A^{*}\right)\right]^{\prime} \times \mathcal{D}\left(A^{*}\right) .
$$

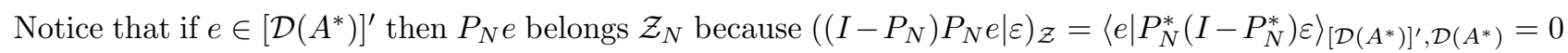
for all $\varepsilon \in \mathcal{D}\left(A^{*}\right)$. Moreover, as in [35] one can prove that $\left[\mathcal{D}\left(A^{*}\right)\right]^{\prime}=\mathcal{Z}_{N} \oplus\left[\mathcal{D}\left(A^{*}\right) \cap \mathcal{Z}_{N}^{*-}\right]^{\prime}$ and since for all $(e, \varepsilon) \in\left[\mathcal{D}\left(A^{*}\right)\right]^{\prime} \times\left(\mathcal{D}\left(A^{*}\right) \cap \mathcal{Z}_{N}^{*-}\right)$ we have:

$$
\left\langle\left(I-P_{N}\right) e \mid \varepsilon\right\rangle_{\left[\mathcal{D}\left(A^{*}\right)\right]^{\prime}, \mathcal{D}\left(A^{*}\right)}=\left\langle e \mid\left(I-P_{N}^{*}\right) \varepsilon\right\rangle_{\left[\mathcal{D}\left(A^{*}\right)\right]^{\prime}, \mathcal{D}\left(A^{*}\right)}=\langle e \mid \varepsilon\rangle_{\left[\mathcal{D}\left(A^{*}\right)\right]^{\prime}, \mathcal{D}\left(A^{*}\right)},
$$

we deduce that the projection operator $\left(I-P_{N}\right)$ can be identified as a bounded operator from $\left[\mathcal{D}\left(A^{*}\right)\right]^{\prime}$ into $\left[\mathcal{D}\left(A^{*}\right) \cap \mathcal{Z}_{N}^{*-}\right]^{\prime}$. As a consequence, for $u \in L_{\text {loc }}^{2}(\mathcal{U})$ we have $P_{N} B u \in L_{\text {loc }}^{2}\left(\mathcal{Z}_{N}\right)$ and $\left(I-P_{N}\right) B u \in L_{l o c}^{2}\left(\left[\mathcal{D}\left(A^{*}\right) \cap\right.\right.$ $\left.\left.\mathcal{Z}_{N}^{*-}\right]^{\prime}\right)$, and by applying $P_{N}$ and $\left(I-P_{N}\right)$ to $(9)$ we deduce that $\left(y_{N}, y_{N}^{-}\right)$obeys

$$
y_{N}^{\prime}=A_{N} y_{N}+P_{N} B u \in \mathcal{Z}_{N} \quad \text { and } \quad y_{N}^{-^{\prime}}=A_{N}^{-} y_{N}^{-}+\left(I-P_{N}\right) B u \in\left[\mathcal{D}\left(A^{*}\right) \cap \mathcal{Z}_{N}^{*-}\right]^{\prime} .
$$

To go further, we introduce the spaces

$$
\mathcal{U}_{N} \stackrel{\text { def }}{=}\left\{B^{*} \varepsilon \mid \varepsilon \in \mathcal{Z}_{N}^{*}\right\} \quad \text { and } \quad \mathcal{U}_{N}^{-} \stackrel{\text { def }}{=}\left\{B^{*} \varepsilon \mid \varepsilon \in \mathcal{D}\left(A^{*}\right) \cap \mathcal{Z}_{N}^{*-}\right\}
$$

as well as the orthogonal projection operators $p_{N}: \mathcal{U} \rightarrow \mathcal{U}_{N}$ and $p_{N}^{-}: \mathcal{U} \rightarrow \mathcal{U}_{N}^{-}$and we recall that their respective adjoints $p_{N}^{*}: \mathcal{U}_{N} \rightarrow \mathcal{U}$ and $p_{N}^{-*}: \mathcal{U}_{N}^{-} \rightarrow \mathcal{U}$ are the injection operators. Thus we introduce the projected control operators:

$$
B_{N} \stackrel{\text { def }}{=} P_{N} B p_{N}^{*}: \mathcal{U}_{N} \longrightarrow \mathcal{Z}_{N} \quad \text { and } \quad B_{N}^{-} \stackrel{\text { def }}{=}\left(I-P_{N}\right) B p_{N}^{*-}: \mathcal{U}_{N}^{-} \longrightarrow\left[\mathcal{D}\left(A^{*}\right) \cap \mathcal{Z}_{N}^{*-}\right]^{\prime}
$$

which obey:

$$
B_{N} p_{N}=P_{N} B \quad \text { and } \quad B_{N}^{-} p_{N}^{-}=\left(I-P_{N}\right) B .
$$

Indeed, the first above equality is obtained by making the following calculations for all $(v, \varepsilon) \in \mathcal{U} \times \mathcal{Z}$ :

$$
\left(P_{N} B v \mid \varepsilon\right)_{\mathcal{Z}}=\left(v \mid B^{*} P_{N}^{*} \varepsilon\right)_{\mathcal{U}}=\left(p_{N} v \mid p_{N} B^{*} P_{N}^{*} \varepsilon\right)_{\mathcal{U}}=\left(P_{N} B p_{N}^{*} p_{N} v \mid \varepsilon\right)_{\mathcal{Z}}=\left(B_{N} p_{N} v \mid \varepsilon\right)_{\mathcal{Z}}
$$


where we have used that $p_{N}$ is an orthogonal projection. The second equality of (17) is obtained by making the following calculations for all $(v, z) \in \mathcal{U} \times \mathcal{D}\left(A^{*}\right)$ :

$$
\begin{aligned}
\left\langle\left(I-P_{N}\right) B v \mid \varepsilon\right\rangle_{\left[\mathcal{D}\left(A^{*}\right)\right]^{\prime}, \mathcal{D}\left(A^{*}\right)} & =\left(v \mid B^{*}\left(I-P_{N}^{*}\right) \varepsilon\right)_{\mathcal{U}}=\left(p_{N}^{-} v \mid p_{N}^{-} B^{*}\left(I-P_{N}^{*}\right) \varepsilon\right)_{\mathcal{U}} \\
& =\left\langle\left(I-P_{N}\right) B p_{N}^{-*} p_{N}^{-} v \mid \varepsilon\right\rangle_{\left[\mathcal{D}\left(A^{*}\right)\right]^{\prime}, \mathcal{D}\left(A^{*}\right)}=\left\langle B_{N}^{-} p_{N}^{-} v \mid \varepsilon\right\rangle_{\left[\mathcal{D}\left(A^{*}\right)\right]^{\prime}, \mathcal{D}\left(A^{*}\right)} .
\end{aligned}
$$

Finally, by combining (15) and (17) we deduce that the pair $\left(y_{N}, y_{N}^{-}\right)$obeys:

$$
\begin{aligned}
y_{N}^{\prime} & =A_{N} y_{N}+B_{N} p_{N} u \in \mathcal{Z}_{N}, \\
y_{N}^{-{ }^{\prime}} & =A_{N}^{-} y_{N}^{-}+B_{N}^{-} p_{N}^{-} u \in\left[\mathcal{D}\left(A^{*}\right) \cap \mathcal{Z}_{N}^{*-}\right]^{\prime} .
\end{aligned}
$$

Notice that conversely, if $\left(y_{N}, y_{N}^{-}\right) \in \mathcal{Z}_{N} \times \mathcal{Z}_{N}^{-}$obeys (18)-(19), then by adding (18) to (19) and by using (17) we recover the fact that $y=y_{N}+y_{N}^{-}$obeys (9). Then we have proved that formulations (9) and (18)-(19) are equivalent, and that the $\mathcal{Z}_{N}$-component (resp. $\mathcal{Z}_{N}^{-}$-component) of the state $y$ is entirely determined by the $\mathcal{U}_{N}$-component (resp. $\mathcal{U}_{N}^{-}$-component) of the control $u$.

Our strategy is to prove the controllability and the stabilization of the finite dimensional system (18). Notice that to achieve this goal, because of the projection $p_{N}$ in the control operator of (18), it is sufficient to take $u \in L^{2}\left(\mathcal{U}_{N}\right)$.

\subsection{Controllability of complex finite dimensional projected system}

We are now interested in constructing a control space of minimal dimension such that the finite dimensional system (18) is exactly controllable. First, let us suppose that $u$ is expressed as a linear combination of $K$ vectors

$$
\left(v_{1}, \ldots, v_{K}\right) \in \mathcal{U}^{K}
$$

as follows:

$$
u=\sum_{j=1}^{K} u_{j} v_{j} \quad \text { where } \quad \bar{u} \stackrel{\text { def }}{=}\left(u_{1}, \ldots, u_{K}\right) \in L^{2}\left(\mathbb{C}^{K}\right) .
$$

Thus, we introduce the linear mapping

$$
V: \mathbb{C}^{K} \longrightarrow\left[\mathcal{D}\left(A^{*}\right)\right]^{\prime}, \quad V \bar{w} \stackrel{\text { def }}{=} \sum_{j=1}^{K} w_{j} B v_{j} \quad \text { where } \quad \bar{w} \stackrel{\text { def }}{=}\left(w_{1}, \ldots, w_{K}\right) \in \mathbb{C}^{K},
$$

and for a control of the form (20) system (9) becomes

$$
y^{\prime}=A y+V \bar{u} \in\left[\mathcal{D}\left(A^{*}\right)\right]^{\prime} .
$$

Notice that the adjoint $V^{*}: \mathcal{D}\left(A^{*}\right) \longrightarrow \mathbb{C}^{K}$ is given by:

$$
V^{*} \varepsilon=\left(\left(v_{j} \mid B^{*} \varepsilon\right) \mathcal{U}\right)_{1 \leq j \leq K}=\left(\left(v_{1} \mid B^{*} \varepsilon\right) \mathcal{U}, \ldots,\left(v_{K} \mid B^{*} \varepsilon\right) \mathcal{U}\right) .
$$

Moreover, by setting $V_{N} \stackrel{\text { def }}{=} P_{N} V$ and $V_{N}^{-\stackrel{\text { def }}{=}}\left(1-P_{N}\right) V$ and recalling (17), system (18)-(19) becomes

$$
\begin{aligned}
y_{N}^{\prime} & =A_{N} y_{N}+V_{N} \bar{u} \in \mathcal{Z}_{N}, \\
y_{N}^{-\prime} & =A_{N}^{-} y_{N}^{-}+V_{N}^{-} \bar{u} \in\left[\mathcal{D}\left(A^{*}\right) \cap \mathcal{Z}_{N}^{*-}\right]^{\prime} .
\end{aligned}
$$

Our main objective here is to characterize the families $\left(v_{j}\right)_{1 \leq j \leq K}$ for which $(24)$ is controllable. According to (18), which states that $y_{N}$ is only determined by $p_{N} u$, if an admissible family $\left(v_{j}\right)_{1 \leq j \leq K}$ exists then it can be chosen in $\left(\mathcal{U}_{N}\right)^{K}$, where $\mathcal{U}_{N}$ is defined by (16). More precisely, we are going to prove that each $v_{j}$ can be chosen in $B^{*}\left(\mathcal{E}_{N}\right)$ where:

$$
\mathcal{E}_{N} \stackrel{\text { def }}{=} \bigoplus_{k=1}^{N} \operatorname{Ker}\left(A^{*}-\bar{\lambda}_{k}\right)=\operatorname{span}_{\mathbb{C}}\left\{\varepsilon_{k}^{i}, k=1, \ldots, N, i=1, \ldots, \ell_{k}\right\},
$$

and that we can choose $K$ equal to the maximal geometric multiplicity of the eigenvalues of $A_{N}$. In the above setting $\left\{\varepsilon_{k}^{i}, i=1, \ldots, \ell_{k}\right\}$ denotes a basis of $\operatorname{Ker}\left(A^{*}-\bar{\lambda}_{k}\right)$ and $\ell_{k}$ is the geometric multiplicity of $\bar{\lambda}_{k}$. Notice that in view of $(13)$ and $(16), B^{*}\left(\mathcal{E}_{N}\right)$ is a subspace of $\mathcal{U}_{N}$ which could be strict, in particular if for some $\lambda_{k}$ the generalized eigenspace $\operatorname{Ker}\left(A^{*}-\bar{\lambda}_{k}\right)^{m_{k}}$ does not coincide with the eigenspace $\operatorname{Ker}\left(A^{*}-\bar{\lambda}_{k}\right)$ i.e. if the geometric multiplicity $\ell_{k}$ is strictly lower that the algebraic multiplicity $\operatorname{dim} \operatorname{Ker}\left(A^{*}-\bar{\lambda}_{k}\right)^{m_{k}}$. Using those notations, the following theorem provides a first necessary and sufficient condition for (24) to be exactly controllable. 
Theorem 3. For all $k=1, \ldots, N$, let $\left\{\varepsilon_{k}^{i}, i=1, \ldots, \ell_{k}\right\}$ be a basis of $\operatorname{Ker}\left(A^{*}-\bar{\lambda}_{k}\right)$, let $\left(v_{j}\right)_{1 \leq j \leq K}$ be a family of $\mathcal{U}^{K}$ and let define:

$$
W_{k} \stackrel{\text { def }}{=}\left(\left(v_{j} \mid B^{*} \varepsilon_{k}^{i}\right)_{\mathcal{U}}\right)_{1 \leq i \leq \ell_{k}, 1 \leq j \leq K}=\left(\begin{array}{ccccc}
\left(v_{1} \mid B^{*} \varepsilon_{k}^{1}\right)_{\mathcal{U}} & \ldots & \left(v_{j} \mid B^{*} \varepsilon_{k}^{1}\right)_{\mathcal{U}} & \ldots & \left(v_{K} \mid B^{*} \varepsilon_{k}^{1}\right)_{\mathcal{U}} \\
\vdots & & \vdots & & \vdots \\
\left(v_{1} \mid B^{*} \varepsilon_{k}^{i}\right)_{\mathcal{U}} & \ldots & \left(v_{j} \mid B^{*} \varepsilon_{k}^{i}\right)_{\mathcal{U}} & \ldots & \left(v_{K} \mid B^{*} \varepsilon_{k}^{i}\right)_{\mathcal{U}} \\
\vdots & & \vdots & & \vdots \\
\left(v_{1} \mid B^{*} \varepsilon_{k}^{\ell_{k}}\right)_{\mathcal{U}} & \ldots & \left(v_{j} \mid B^{*} \varepsilon_{k}^{\ell_{k}}\right)_{\mathcal{U}} & \ldots & \left(v_{K} \mid B^{*} \varepsilon_{k}^{\ell_{k}}\right)_{\mathcal{U}}
\end{array}\right) .
$$

Then the system (24) is exactly controllable, if and only if, for all $k=1, \ldots, N, \operatorname{Rank}\left(W_{k}\right)=\ell_{k}$.

Proof. Because $\mathcal{Z}_{N}$ is finite dimensional, (24) is exactly controllable, if and only if, the following Hautus test is satisfied, see [39, Prop. 1.5.5]:

$$
\forall k \in\{1, \ldots, N\}, \quad \operatorname{Rank}\left[A_{N}-\lambda_{k} \mid V_{N}\right]=\operatorname{dim}\left(\mathcal{Z}_{N}\right),
$$

or equivalently:

$$
\forall k \in\{1, \ldots, N\}, \quad \mathcal{Z}_{N}=\left\{\left(A_{N}-\lambda_{k}\right) e+V_{N} \bar{u} \mid(e, \bar{u}) \in \mathcal{Z}_{N} \times \mathbb{C}^{K}\right\} .
$$

Thus, we have that (24) is exactly controllable, if and only if, for all $\varepsilon \in \mathcal{Z}_{N}^{*}$ and all $k=1, \ldots, N$ we have :

$$
\forall(e, \bar{u}) \in \mathcal{Z}_{N} \times \mathbb{C}^{K}, \quad\left(\left(A_{N}-\lambda_{k}\right) e+V_{N} \bar{u} \mid \varepsilon\right)_{\mathcal{Z}}=0 \quad \Longrightarrow \quad \varepsilon=0 .
$$

Indeed, the fact that (26) implies (27) follows from the first equality in (14) which guarantees that $\mathcal{Z}_{N}^{\perp} \cap \mathcal{Z}_{N}^{*}=\{0\}$. Conversely, if (26) is not satisfied, then there exists a nonzero $e_{0} \in \mathcal{Z}_{N}$ which is orthogonal to the space defined at the right of the equality in (26), and we verify that (27) is false for $\varepsilon=P_{N}^{*} e_{0} \in \mathcal{Z}_{N}^{*}$ (which is also non null because it obeys $\left.\left(e_{0} \mid \varepsilon\right)_{\mathcal{Z}}=\left\|e_{0}\right\|_{\mathcal{Z}}^{2}\right)$.

Next, by replacing $e \in \mathcal{Z}_{N}$ by $P_{N} e$ for $e \in \mathcal{Z}$ in $(27)$ and since $V_{N} \bar{u}=P_{N} V \bar{u}$ for all $\bar{u} \in \mathbb{C}^{K}$, we obtain that for $\varepsilon \in \mathcal{Z}_{N}^{*},(27)$ is also equivalent to:

$$
\forall(e, \bar{u}) \in \mathcal{Z} \times \mathbb{C}^{K}, \quad\left(e \mid\left(A^{*}-\bar{\lambda}_{k}\right) \varepsilon\right)_{\mathcal{Z}}=0 \quad \text { and } \quad\left(\bar{u} \mid V^{*} \varepsilon\right)_{\mathbb{C}^{K}}=0 \quad \Longrightarrow \quad \varepsilon=0 .
$$

According to (23), we have proved that (24) is exactly controllable, if and only if, for all $k \in\{1, \ldots, N\}$ we have

$$
\forall j \in\{1, \ldots, K\}, \forall \varepsilon \in \operatorname{Ker}\left(A^{*}-\bar{\lambda}_{k}\right), \quad\left(v_{j} \mid B^{*} \varepsilon\right)_{\mathcal{U}}=0 \quad \Longrightarrow \quad \varepsilon=0 .
$$

Finally, because the above implication means that $\operatorname{Ker}\left({ }^{t} W_{k}\right)=\{0\}$, the desired result is obtained.

The two first obvious consequences of the above theorem is that $K$ must be greater or equal to all the geometric multiplicities $\ell_{k}$, and that there is no eigenvector related to an eigenvalue $\bar{\lambda}_{k}$ of $A^{*}$ which is in the kernel of $B^{*}$. Otherwise there would be a linear combination of lines of $W_{k}$ equal to zero for some $k \in\{1, \ldots, N\}$.

Corollary 4. The following conditions are necessary for the exact controllability of (24):

1. Any $\varepsilon \in \mathcal{D}\left(A^{*}\right)$ which obeys $A^{*} \varepsilon=\bar{\lambda} \varepsilon$ and $B^{*} \varepsilon=0$ for $\lambda \in \Sigma_{N}$ is necessarily equal to zero.

2. $K$ is greater or equal to the maximum of the geometric multiplicities of $\lambda_{k}, k=1, \ldots, N$ :

$$
K \geq \max \left\{\ell_{k} \mid k=1, \ldots, N\right\} .
$$

Let us remark that Condition 1 of the above corollary can be stated in the following equivalent way:

1'. For all $k=1, \ldots, N$ the family $B^{*} \varepsilon_{k}^{i} i=1, \ldots, \ell_{k}$ is linearly independent.

The pertinent question is then whether or not Conditions 1 and 2 are also sufficient. Notice that a first obvious sufficient condition is that $K=\max \left(\ell_{k}\right)$ and the whole family $B^{*} \varepsilon_{k}^{i}, i=1, \ldots, \ell_{k} ; k=1, \ldots, N$ is linearly independent. Indeed, in such a situation it is easily seen that one can choose $\left(v_{1}, \ldots, v_{K}\right)$ in $B^{*}\left(\mathcal{E}_{N}\right)^{K}$ such that each matrix $W_{k}$ coincides with any prescribed element of $\mathbb{C}^{\ell_{k} \times K}$. However, although the linear independence of $B^{*} \varepsilon_{k}^{i}, i=1, \ldots, \ell_{k} ; k=1, \ldots, N$ is true in the particular case of internal control (see [8,4]) it may be false for Dirichlet boundary control in some simple situations as it has been pointed out in the introduction. Another sufficient condition is $K=\sum_{k=1}^{N} \ell_{k}$ with Condition 1 (or equivalently $1^{\prime}$ ): if we choose $\left(v_{1}, \ldots, v_{K}\right)$ to be the whole family $\left(B^{*} \varepsilon_{1}^{1}, \ldots, B^{*} \varepsilon_{N}^{\ell_{N}}\right)$ then each matrix $W_{k}$ contains a block $\left(\left(B^{*} \varepsilon_{k}^{j} \mid B^{*} \varepsilon_{k}^{i}\right)_{\mathcal{U}}\right)_{1 \leq i, j \leq \ell_{k}}$ which is invertible since $B^{*} \varepsilon_{k}^{i} i=1, \ldots, \ell_{k}$ is linearly independent. In fact, Conditions 1 and 2 of Corollary 4 are also sufficient to construct $K$ vectors $v_{1}, \ldots, v_{K}$ of $B^{*}\left(\mathcal{E}_{N}\right)$ such that $(24)$ is exactly controllable. 
Theorem 5. Assume Conditions 1 and 2 of Corollary 4 hold and in particular

$$
K \geq \max \left\{\ell_{k} \mid k=1, \ldots, N\right\} .
$$

Then there exists a family $\left(v_{j}\right)_{1 \leq j \leq K}$ of $B^{*}\left(\mathcal{E}_{N}\right)$ such that $(24)$ is exactly controllable. Moreover, the families $\left(v_{j}\right)_{1 \leq j \leq K}$ such that $(24)$ is exactly controllable form an open and dense subset of $\mathcal{U}^{K}$.

Proof. First, for all $k \in\{1, \ldots, N\}$ let $\mathcal{M}_{\ell_{k}, K}(\mathbb{C})$ be the space of complex matrix of size $\ell_{k} \times K$ and let us consider the subset $\mathcal{R}_{k}$ of $\mathcal{M}_{\ell_{k}, K}(\mathbb{C})$ composed by the matrices of rank $\ell_{k}$. We also consider the continuous linear maps

$$
\Theta_{k}: \mathcal{U}^{K} \rightarrow \mathcal{M}_{\ell_{k}, K}(\mathbb{C}), \quad\left(v_{1}, \ldots, v_{K}\right) \mapsto W_{k}\left(v_{1}, \ldots, v_{K}\right) \stackrel{\text { def }}{=}\left(\left(v_{j} \mid B^{*} \varepsilon_{k}^{i}\right) \mathcal{U}\right)_{1 \leq i \leq \ell_{k}, 1 \leq j \leq K}
$$

By using Theorem 3, we know that the set of admissible families

$$
\mathcal{A} \stackrel{\text { def }}{=}\left\{\left(v_{j}\right)_{1 \leq j \leq K} \in \mathcal{U}^{K} \mid(24) \text { is exactly controllable }\right\}
$$

is characterized by

$$
\mathcal{A}=\bigcap_{k=1}^{N} \Theta_{k}^{-1}\left(\mathcal{R}_{k}\right)
$$

Since $\mathcal{R}_{k}$ is an open subset of $\mathcal{M}_{\ell_{k}, K}(\mathbb{C})$, we deduce that $\Theta_{k}^{-1}\left(\mathcal{R}_{k}\right)$ is an open subset of $\mathcal{U}^{K}$ and thus $\mathcal{A}$ is also an open subset of $\mathcal{U}^{K}$.

Second, let set $\ell \stackrel{\text { def }}{=} \sum_{r=1}^{N} \ell_{r}$, let denote by $v(z) \stackrel{\text { def }}{=} \sum_{k=1}^{N} \sum_{i=1}^{\ell_{k}} z_{k, i} B^{*} \varepsilon_{k}^{i}$ where $z \stackrel{\text { def }}{=}\left(z_{k, i}\right)_{k=1, \ldots, N, i=1, \ldots, \ell_{k}} \in \mathbb{C}^{\ell}$ the elements of $B^{*}\left(\mathcal{E}_{N}\right)$ :

$$
B^{*}\left(\mathcal{E}_{N}\right)=\left\{v(z) \mid z \in \mathbb{C}^{\ell}\right\}
$$

and let denote by $U(Z) \stackrel{\text { def }}{=}\left(v\left(z^{(1)}\right), \ldots, v\left(z^{(K)}\right)\right) \in\left(B^{*}\left(\mathcal{E}_{N}\right)\right)^{K}$ where $Z \stackrel{\text { def }}{=}\left(z^{(1)}, \ldots, z^{(K)}\right) \in \mathbb{C}^{\ell \times K}$ the elements of $\left(B^{*}\left(\mathcal{E}_{N}\right)\right)^{K}$ :

$$
\left(B^{*}\left(\mathcal{E}_{N}\right)\right)^{K}=\left\{U(Z) \mid Z \in \mathbb{C}^{\ell \times K}\right\}
$$

If we denote by $B^{*}\left(\mathcal{E}_{N}\right)^{\perp}$ the orthogonal of $B^{*}\left(\mathcal{E}_{N}\right)$ in $\mathcal{U}$, then the following bounded linear mapping is onto:

$$
\Psi:\left(B^{*}\left(\mathcal{E}_{N}\right)^{\perp}\right)^{K} \times \mathbb{C}^{\ell \times K} \mapsto \mathcal{U}^{K}, \quad \Psi\left(U_{\perp}, Z\right) \stackrel{\text { def }}{=} U_{\perp}+U(Z) .
$$

Moreover, Condition 2 of Corollary 4 ensures that for $k=1, \ldots, N$ and $Z \in \mathbb{C}^{\ell \times K}$ the following determinant is well-defined:

$$
Q_{k}(Z) \stackrel{\text { def }}{=} \operatorname{det}\left(\begin{array}{ccccc}
\left(v\left(z^{(1)}\right) \mid B^{*} \varepsilon_{k}^{1}\right) \mathcal{U} & \ldots & \left(v\left(z^{(j)}\right) \mid B^{*} \varepsilon_{k}^{1}\right) \mathcal{U} & \ldots & \left(v\left(z^{\left(\ell_{k}\right)}\right) \mid B^{*} \varepsilon_{k}^{1}\right) \mathcal{U} \\
\vdots & & \vdots & & \vdots \\
\left(v\left(z^{(1)}\right) \mid B^{*} \varepsilon_{k}^{i}\right) \mathcal{U} & \ldots & \left(v\left(z^{(j)}\right) \mid B^{*} \varepsilon_{k}^{i}\right) \mathcal{U} & \ldots & \left(v\left(z^{\left(\ell_{k}\right)}\right) \mid B^{*} \varepsilon_{k}^{i}\right) \mathcal{U} \\
\vdots & & \vdots & & \vdots \\
\left(v\left(z^{(1)}\right) \mid B^{*} \varepsilon_{k}^{\ell_{k}}\right) \mathcal{U} & \ldots & \left(v\left(z^{(j)}\right) \mid B^{*} \varepsilon_{k}^{\ell_{k}}\right) \mathcal{U} & \ldots & \left(v\left(z^{\left(\ell_{k}\right)}\right) \mid B^{*} \varepsilon_{k}^{\ell_{k}}\right) \mathcal{U}
\end{array}\right),
$$

and we set

$$
Q(Z) \stackrel{\text { def }}{=} \prod_{k=1}^{N} Q_{k}(Z)
$$

Thus, we denote by $\mathbb{O}$ the set of roots of $Q$ and by $\mathbb{O}^{c}$ its complementary part in $\mathbb{C}^{\ell \times K}$. Then we have:

$$
\mathcal{A} \supset\left\{U_{\perp}+U(Z) \mid\left(U_{\perp}, Z\right) \in\left(B^{*}\left(\mathcal{E}_{N}\right)^{\perp}\right)^{K} \times \mathbb{C}^{\ell \times K}, Q(Z) \neq 0\right\}=\Psi\left(\left(B^{*}\left(\mathcal{E}_{N}\right)^{\perp}\right)^{K} \times \mathbb{O}^{c}\right) .
$$

We observe that for all $k=1, \ldots, N$ the coefficient of $z_{k, 1}^{(1)} z_{k, 2}^{(2)} \ldots z_{k, \ell_{k}}^{\left(\ell_{k}\right)}$ in $Q_{k}(Z)$ is equal to

$$
\operatorname{det}\left(\begin{array}{ccccc}
\left(B^{*} \varepsilon_{k}^{1} \mid B^{*} \varepsilon_{k}^{1}\right) \mathcal{U} & \ldots & \left(B^{*} \varepsilon_{k}^{j} \mid B^{*} \varepsilon_{k}^{1}\right) \mathcal{U} & \ldots & \left(B^{*} \varepsilon_{k}^{\ell_{k}} \mid B^{*} \varepsilon_{k}^{1}\right) \mathcal{U} \\
\vdots & & \vdots & & \vdots \\
\left(B^{*} \varepsilon_{k}^{1} \mid B^{*} \varepsilon_{k}^{i}\right) \mathcal{U} & \ldots & \left(B^{*} \varepsilon_{k}^{j} \mid B^{*} \varepsilon_{k}^{i}\right) \mathcal{U} & \ldots & \left(B^{*} \varepsilon_{k}^{\ell_{k}} \mid B^{*} \varepsilon_{k}^{i}\right) \mathcal{U} \\
\vdots & & \vdots & & \vdots \\
\left(B^{*} \varepsilon_{k}^{1} \mid B^{*} \varepsilon_{k}^{\ell_{k}}\right) \mathcal{U} & \ldots & \left(B^{*} \varepsilon_{k}^{j} \mid B^{*} \varepsilon_{k}^{\ell_{k}}\right) \mathcal{U} & \ldots & \left(B^{*} \varepsilon_{k}^{\ell_{k}} \mid B^{*} \varepsilon_{k}^{\ell_{k}}\right) \mathcal{U}
\end{array}\right) .
$$


By remarking that Condition 1 of Corollary 4 ensures that $\left\{B^{*} \varepsilon_{k}^{i} \mid i=1, \ldots, \ell_{k}\right\}$ is a linearly independent family, we deduce that the above determinant is not zero and then that the polynomials $Q_{k}$ for all $k=1, \ldots, N$ are not identically zero. As a consequence, $Q \neq 0$ and the complementary set of its roots $\mathbb{O}^{c}$ is a dense subset of $\mathbb{C}^{\ell \times K}$. Then the density of $\Psi\left(\left(B^{*}\left(\mathcal{E}_{N}\right)^{\perp}\right)^{K} \times \mathbb{O}^{c}\right)$ in $\mathcal{U}^{K}$ follows from the continuity and the surjectivity of the linear map $\Psi$. It implies the density of $\mathcal{A}$. Finally, the existence of a family $\left(v_{j}\right)_{1 \leq j \leq K}$ of $B^{*}\left(\mathcal{E}_{N}\right)$ such that (24) is exactly controllable can be proved by considering $\Psi((0, Z))$ where $Z$ is such that $Q(Z) \neq 0$.

Finally, let us give a sufficient condition for Condition 1 of Corollary 4.

Theorem 6. If system (9) is approximately controllable, then any $\varepsilon \in \mathcal{D}\left(A^{*}\right)$ which obeys $A^{*} \varepsilon=\lambda \varepsilon$ and $B^{*} \varepsilon=0$ for $\lambda \in \mathbb{C}$ is necessarily equal to zero.

Proof. Since the proof is obvious for $\lambda$ in the resolvent set of $A^{*}$, we can suppose that $\lambda$ is an eigenvalue of $A^{*}$. Assume that $\varepsilon \in \operatorname{Ker}\left(A^{*}-\lambda\right)$ and $B^{*} \varepsilon=0$ and let us prove that $\varepsilon \equiv 0$. By following the lines of Subsection 3.1 we define a projection operator $P_{\bar{\lambda}}: \mathcal{Z} \rightarrow \mathcal{Z}$ by:

$$
P_{\bar{\lambda}} \stackrel{\text { def }}{=}-\frac{1}{2 \pi \imath} \int_{\Gamma_{\bar{\lambda}}}(\xi-A)^{-1} d \xi,
$$

where $\Gamma_{\bar{\lambda}}$ is now a positively-oriented curve enclosing $\bar{\lambda}$ but no other point of the spectrum of $A$. We then define the invariant subspaces $\mathcal{Z}_{\bar{\lambda}} \stackrel{\text { def }}{=} P_{\bar{\lambda}}(\mathcal{Z})$ and $\mathcal{Z}_{\bar{\lambda}}^{*} \stackrel{\text { def }}{=} P_{\bar{\lambda}}^{*}(\mathcal{Z})$ of $A$ and $A^{*}$ respectively as well as the finite dimensional restricted operator $\left.A_{\bar{\lambda}} \stackrel{\text { def }}{=} A\right|_{\mathcal{Z}_{\bar{\lambda}}}$, with spectrum reduced to $\{\bar{\lambda}\}$. We also introduce $B_{\bar{\lambda}} \stackrel{\text { def }}{=} P_{\bar{\lambda}} B p_{\bar{\lambda}}^{*}$, where $p_{\bar{\lambda}}$ is the orthogonal projection operator from $\mathcal{U}$ onto $\mathcal{U}_{\bar{\lambda}} \stackrel{\text { def }}{=}\left\{B^{*} \varepsilon \mid \varepsilon \in \mathcal{Z}_{\bar{\lambda}}^{*}\right\}$ and by applying $P_{\bar{\lambda}}$ to $(9)$ we obtain that $y_{\bar{\lambda}} \stackrel{\text { def }}{=} P_{\bar{\lambda}} y$ and $u_{\bar{\lambda}} \stackrel{\text { def }}{=} p_{\bar{\lambda}} u$ obey:

$$
y_{\bar{\lambda}}^{\prime}=A_{\bar{\lambda}} y_{\bar{\lambda}}+B_{\bar{\lambda}} u_{\bar{\lambda}}
$$

Because the approximate controllability of (9) implies the approximate controllability of (30) and because (30) is a finite dimensional system, we deduce that (30) is exactly controllable. As a consequence, the Hautus test $\operatorname{Rank}\left[A_{\bar{\lambda}}-\bar{\lambda} \mid B_{\bar{\lambda}}\right]=\operatorname{dim}\left(\mathcal{Z}_{\bar{\lambda}}\right)$ is satisfied, which means that for all $\widetilde{\varepsilon} \in \mathcal{Z}_{\bar{\lambda}}^{*}$ :

$$
\forall(e, u) \in \mathcal{Z}_{\bar{\lambda}} \times \mathcal{U}_{\bar{\lambda}} \quad\left(\left(A_{\bar{\lambda}}-\lambda\right) e+B_{\bar{\lambda}} u \mid \widetilde{\varepsilon}\right)_{\mathcal{Z}}=0 \quad \Longrightarrow \quad \widetilde{\varepsilon}=0
$$

or equivalently

$$
\forall(e, u) \in \mathcal{Z}_{\bar{\lambda}} \times \mathcal{U}_{\bar{\lambda}} \quad\left(e \mid\left(A^{*}-\lambda\right) \widetilde{\varepsilon}\right)_{\mathcal{Z}}=0 \quad \text { and } \quad\left(u \mid B^{*} \widetilde{\varepsilon}\right)_{\mathcal{U}}=0 \quad \Longrightarrow \quad \widetilde{\varepsilon}=0 .
$$

Finally, since $\varepsilon \in \operatorname{Ker}\left(A^{*}-\lambda\right) \subset \mathcal{Z}_{\bar{\lambda}}^{*}$ and $B^{*} \varepsilon=0$, we obtain $\varepsilon \equiv 0$ from the above implication with $\widetilde{\varepsilon}=\varepsilon$.

\subsection{Controllability of real finite dimensional projected system}

Let us now consider $A$ and $B$ as operators on the real Hilbert spaces $\mathcal{X}$ and $\mathcal{V}: A: \mathcal{D}(A) \subset \mathcal{X} \rightarrow \mathcal{X}$ and $B \in \mathcal{L}\left(\mathcal{V},\left[\mathcal{D}\left(A^{*}\right)\right]^{\prime}\right)$. We can use the results of the previous subsection by considering $A$ and $B$ as operators in the complexified spaces $\mathcal{Z}$ and $\mathcal{U}$ (see Section 2). Since the spectrum of $A$ admits the real line as a symmetry axis, the positively-oriented curve $\Gamma_{N}$ in (12) can be defined so that it obeys $\Gamma_{N}=\bar{\Gamma}_{N}$, and an easy calculation shows that it implies $P_{N}=\bar{P}_{N}$. As a consequence, the projection operator $P_{N}$ maps $\mathcal{X}$ to itself and it provides the following decompositions of $\mathcal{X}$ :

$$
\mathcal{X}=\mathcal{X}_{N} \oplus \mathcal{X}_{N}^{-}, \quad \mathcal{X}_{N} \stackrel{\text { def }}{=} P_{N}(\mathcal{X}) \quad \text { and } \quad \mathcal{X}_{N}^{-} \stackrel{\text { def }}{=}\left(I-P_{N}\right)(\mathcal{X})
$$

and

$$
\mathcal{X}=\mathcal{X}_{N}^{*} \oplus \mathcal{X}_{N}^{*-}, \quad \mathcal{X}_{N}^{*} \stackrel{\text { def }}{=} P_{N}^{*}(\mathcal{X}) \quad \text { and } \quad \mathcal{X}_{N}^{*-} \stackrel{\text { def }}{=}\left(I-P_{N}^{*}\right)(\mathcal{X}) .
$$

Analogously as in Subsection 3.1, we introduce the real control spaces

$$
\mathcal{V}_{N} \stackrel{\text { def }}{=}\left\{B^{*} x \mid x \in \mathcal{X}_{N}^{*}\right\} \quad \text { and } \quad \mathcal{V}_{N}^{-} \stackrel{\text { def }}{=}\left\{B^{*} x \mid x \in \mathcal{D}\left(A^{*}\right) \cap \mathcal{X}_{N}^{*-}\right\},
$$

with their related orthogonal projections $p_{N}: \mathcal{V} \rightarrow \mathcal{V}_{N}$ and $p_{N}^{-}: \mathcal{V} \rightarrow \mathcal{V}_{N}^{-}$and control operators:

$$
B_{N} \stackrel{\text { def }}{=} P_{N} B p_{N}^{*}: \mathcal{V}_{N} \longrightarrow \mathcal{X}_{N} \quad \text { and } \quad B_{N}^{-} \stackrel{\text { def }}{=}\left(I-P_{N}\right) B p_{N}^{-*}: \mathcal{V}_{N}^{-} \longrightarrow\left[\mathcal{D}\left(A^{*}\right) \cap \mathcal{X}_{N}^{*-}\right]^{\prime}
$$


We recall that the adjoints $p_{N}^{*}: \mathcal{V}_{N} \rightarrow \mathcal{V}$ and $p_{N}^{-*}: \mathcal{V}_{N}^{-} \rightarrow \mathcal{V}$ are simply injection operators. Thus, by following the steps of Subsection 3.1 it can be shown that for $u \in L^{2}(0, T ; \mathcal{V}), y$ is solution of (9) if and only if the pair $\left(y_{N}, y_{N}^{-}\right) \stackrel{\text { def }}{=}\left(P_{N} y,\left(I-P_{N}\right) y\right)$ satisfies:

$$
\begin{aligned}
y_{N}^{\prime} & =A_{N} y_{N}+B_{N} p_{N} u \in \mathcal{X}_{N}, \\
y_{N}^{-{ }^{\prime}} & =A_{N}^{-} y_{N}^{-}+B_{N}^{-} p_{N}^{-} u \in\left[\mathcal{D}\left(A^{*}\right) \cap \mathcal{X}_{N}^{*-}\right]^{\prime}
\end{aligned}
$$

Next, we denote:

$$
\mathcal{F}_{N} \stackrel{\text { def }}{=} \operatorname{span}_{\mathbb{R}}\left\{\Re \varepsilon_{k}^{i}, \Im \varepsilon_{k}^{i}, k=1, \ldots, N, i=1, \ldots \ell_{k}\right\},
$$

and the following theorem states that $\left(v_{j}\right)_{1 \leq j \leq K}$ provided by Theorem 5 can be chosen in $\left(B^{*}\left(\mathcal{F}_{N}\right)\right)^{K} \subset\left(\mathcal{V}_{N}\right)^{K}$.

Theorem 7. If Conditions 1 and 2 of Corollary 4 hold then there exists a family $\left(v_{j}\right)_{1 \leq j \leq K}$ of $B^{*}\left(\mathcal{F}_{N}\right)$ such that (31) is exactly controllable. Moreover, the families $\left(v_{j}\right)_{1 \leq j \leq K}$ such that $(31)$ is exactly controllable form an open and dense subset of $\mathcal{V}^{K}$.

Proof. The proof that

$$
\mathcal{A} \stackrel{\text { def }}{=}\left\{\left(v_{j}\right)_{1 \leq j \leq K} \in \mathcal{V}^{K} \mid(31) \text { is exactly controllable }\right\}
$$

is an open subset of $\mathcal{V}^{K}$ is similar to the proof of Theorem 5 and we skip it. For the density of $\mathcal{A}$, we also follow the same arguments than in the proof of Theorem 5: we set $\ell \stackrel{\text { def }}{=} \sum_{k=1}^{N} \ell_{k}$ and we define

$$
v(x, y) \stackrel{\text { def }}{=} \sum_{k, i} x_{k, i} B^{*} \Re \varepsilon_{k}^{i}+\sum_{k, i} y_{k, i} B^{*} \Im \varepsilon_{k}^{i}, \quad(x, y) \stackrel{\text { def }}{=}\left(x_{k, i}, y_{k, i}\right)_{k=1, \ldots, N, i=1, \ldots, \ell_{k}} \in \mathbb{R}^{2 \ell},
$$

and

$$
U(X, Y) \stackrel{\text { def }}{=}\left(v\left(x^{(1)}, y^{(1)}\right), \ldots, v\left(x^{(K)}, y^{(K)}\right)\right), \quad(X, Y) \stackrel{\text { def }}{=}\left(x^{(1)}, \ldots, x^{(K)}, y^{(1)}, \ldots, y^{(K)}\right) \in \mathbb{R}^{2 \ell \times K}
$$

In particular, we have

$$
B^{*}\left(\mathcal{F}_{N}\right)=\left\{v(x, y) \mid(x, y) \in \mathbb{R}^{2 \ell}\right\}
$$

and the linear and continuous map

$$
\Phi:\left(B^{*}\left(\mathcal{F}_{N}\right)^{\perp}\right)^{K} \times \mathbb{R}^{2 \ell \times K} \rightarrow \mathcal{V}^{K}: \Phi\left(V^{\perp}, X, Y\right) \stackrel{\text { def }}{=} V^{\perp}+U(X, Y)
$$

is onto.

Next, Condition 2 of Corollary 4 ensures that for $k=1, \ldots, N$ the following determinant is well-defined:

$$
R_{k}(X, Y) \stackrel{\text { def }}{=} \operatorname{det}\left(\begin{array}{ccccc}
\left(v\left(x^{(1)}, y^{(1)}\right) \mid B^{*} \varepsilon_{k}^{1}\right) \mathcal{U} & \ldots & \left(v\left(x^{(j)}, y^{(j)}\right) \mid B^{*} \varepsilon_{k}^{1}\right) \mathcal{U} & \ldots & \left(v\left(x^{\left(\ell_{k}\right)}, y^{\left(\ell_{k}\right)}\right) \mid B^{*} \varepsilon_{k}^{1}\right) \mathcal{U} \\
\vdots & & \vdots & \vdots \\
\left(v\left(x^{(1)}, y^{(1)}\right) \mid B^{*} \varepsilon_{k}^{i}\right) \mathcal{U} & \ldots & \left(v\left(x^{(j)}, y^{(j)}\right) \mid B^{*} \varepsilon_{k}^{i}\right) \mathcal{U} & \ldots & \left(v\left(x^{\left(\ell_{k}\right)}, y^{\left(\ell_{k}\right)}\right) \mid B^{*} \varepsilon_{k}^{i}\right) \mathcal{U} \\
\vdots & & \vdots & \vdots \\
\left(v\left(x^{(1)}, y^{(1)}\right) \mid B^{*} \varepsilon_{k}^{\ell_{k}}\right) \mathcal{U} & \ldots & \left(v\left(x^{(j)}, y^{(j)}\right) \mid B^{*} \varepsilon_{k}^{\ell_{k}}\right) \mathcal{U} & \ldots & \left(v\left(x^{\left(\ell_{k}\right)}, y^{\left(\ell_{k}\right)}\right) \mid B^{*} \varepsilon_{k}^{\ell_{k}}\right) \mathcal{U}
\end{array}\right),
$$

and we set

$$
R(X, Y) \stackrel{\text { def }}{=} \prod_{k=1}^{N} R_{k}(X, Y)
$$

We denote by $\mathbb{O}_{r}$ the set of real roots of $R$ and by $\mathbb{O}_{r}^{c}$ its complementary part in $\mathbb{R}^{2 \ell \times K}$. By applying Theorem 3 , we have

$$
\mathcal{A} \supset \Phi\left(\left(B^{*}\left(\mathcal{F}_{N}\right)^{\perp}\right)^{K} \times \mathbb{O}_{r}^{c}\right)
$$

Next, we remark that for $Z \in \mathbb{C}^{\ell}$ we have $R(Z, \imath Z)=Q(Z)$, where $Q$ is defined by (28). By using Condition 1 of Corollary $4, Q \neq 0$ and thus $R \neq 0$. Thus $\mathbb{O}_{r}^{c}$ is dense in $\mathbb{R}^{2 \ell \times K}$ and we deduce that $\Phi\left(\left(B^{*}\left(\mathcal{F}_{N}\right)^{\perp}\right)^{K} \times \mathbb{O}_{r}^{c}\right)$ is dense in $\mathcal{V}^{K}$. 
In the following we consider a real family $\left(v_{1}, \ldots, v_{K}\right)$ provided by Theorem 7 and the associated linear coordinate mapping

$$
V: \mathbb{R}^{K} \longrightarrow\left[\mathcal{D}\left(A^{*}\right)\right]^{\prime}, \quad V \bar{w} \stackrel{\text { def }}{=} \sum_{j=1}^{K} w_{j} B v_{j} \quad \text { where } \quad \bar{w}=\left(w_{1}, \ldots, w_{K}\right) \in \mathbb{R}^{K} .
$$

The mapping $V_{N} \stackrel{\text { def }}{=} P_{N} V$ now maps $\mathbb{R}^{K}$ into $\mathcal{X}_{N}$ and (31) with

$$
u=\sum_{j=1}^{K} u_{j} v_{j} \quad \text { where } \quad \bar{u}=\left(u_{1}, \ldots, u_{K}\right) \in L^{2}\left(\mathbb{R}^{K}\right)
$$

becomes the following real dynamical system:

$$
y_{N}^{\prime}=A_{N} y_{N}+V_{N} \bar{u} \in \mathcal{X}_{N}
$$

Notice that the exact controllability of (34) is a straightforward consequence of the exact controllability of (24) which is provided by Theorem 7 .

\subsection{Feedback stabilization of real finite-dimensional projected system}

Let us assume that Conditions 1 and 2 of Corollary 4 hold and let us consider the real family $\left(v_{j}\right)_{1 \leq j \leq K}$ given by Theorem 7. The main goal of this subsection is to prove that for $\sigma>0$ satisfying (10), system (34) is stabilizable with a rate of decay $-\sigma$, by means of a linear feedback controller. First, from (34) the change of variable $\left(y_{N, \sigma}(t), \bar{u}_{\sigma}(t)\right)=e^{\sigma t}\left(y_{N}(t), \bar{u}(t)\right)$ yields the system:

$$
y_{N, \sigma}^{\prime}=\left(A_{N}+\sigma\right) y_{N, \sigma}+V_{N} \bar{u}_{\sigma} \in \mathcal{X}_{N},
$$

which is exactly controllable since (34) is exactly controllable. Thus, the following optimal control problem is well-posed:

$$
\inf \left\{\frac{1}{2} \int_{0}^{\infty}\left\|y_{N, \sigma}\right\|_{\mathcal{X}}^{2} d t+\frac{1}{2} \int_{0}^{\infty}\left\|\bar{u}_{\sigma}\right\|_{\mathbb{R}^{K}}^{2} d t ;\left(y_{N, \sigma}, \bar{u}_{\sigma}\right) \text { obeys }(35) \text { and } y_{N, \sigma}(0)=h\right\},
$$

and from Theorem 2.9 p. 44, Theorem 1.4 p. 136 and Theorem 1.5 p. 138 in [41] we know that the solution to the above optimal control problem is exponentially stable and obeys the feedback equality $\bar{u}_{\sigma}(t)=-V_{N}^{*} \widetilde{\Pi} y_{N, \sigma}(t)$ where $V_{N}^{*}: \mathcal{X}_{\mathcal{N}} \rightarrow \mathbb{R}^{K}$ is the adjoint of $V_{N}$ and $\widetilde{\Pi} \in \mathcal{L}\left(\mathcal{X}_{N}\right)$ is the unique solution of the Riccati equation: for all $(\xi, \zeta) \in \mathcal{X}_{N} \times \mathcal{X}_{N}$

$$
\begin{aligned}
& (\xi \mid \zeta)_{\mathcal{X}}+\left(\left(A_{N}+\sigma\right) \xi \mid \widetilde{\Pi} \zeta\right)_{\mathcal{X}}+\left(\widetilde{\Pi} \xi \mid\left(A_{N}+\sigma\right) \zeta\right)_{\mathcal{X}}-\left(V_{N}^{*} \widetilde{\Pi} \xi \mid V_{N}^{*} \widetilde{\Pi} \zeta\right)_{\mathbb{R}^{K}}=0, \\
& (\widetilde{\Pi} \xi \mid \zeta)_{\mathcal{X}}=(\xi \mid \widetilde{\Pi} \zeta)_{\mathcal{X}} \quad \text { and } \quad(\widetilde{\Pi} \xi \mid \xi)_{\mathcal{X}}>0 \text { if } \xi \neq 0 .
\end{aligned}
$$

Notice that as it is underlined in [35, Par. 5], the solution $\widetilde{\Pi}$ belongs to $\mathcal{L}\left(\mathcal{X}_{N}\right)$ because when we have applied the theory of [41] we have identified $\mathcal{X}_{N}$ with its dual. However, we remark that $\widetilde{\Pi}$ is simply the orthogonal projection of $\Pi \stackrel{\text { def }}{=} P_{N}^{*} \widetilde{\Pi} \in \mathcal{L}\left(\mathcal{X}_{N}, \mathcal{X}_{N}^{*}\right)$ on $\mathcal{X}_{\mathcal{N}}:(\Pi \xi \mid \zeta)_{\mathcal{X}}=(\widetilde{\Pi} \xi \mid \zeta) \mathcal{X}$ for all $(\xi, \zeta) \in \mathcal{X}_{N} \times \mathcal{X}_{N}$. Then by observing that we have $V_{N}^{*} \widetilde{\Pi}=V^{*} P_{N}^{*} \widetilde{\Pi}=V^{*} \Pi$, where $V^{*}: \mathcal{D}\left(A^{*}\right) \rightarrow \mathbb{R}^{K}$ is the adjoint of $V$ given in (23), we deduce that $\Pi$ is the unique element of $\mathcal{L}\left(\mathcal{X}_{N}, \mathcal{X}_{N}^{*}\right)$ which obeys: for all $(\xi, \zeta) \in \mathcal{X}_{N} \times \mathcal{X}_{N}$,

$$
\begin{aligned}
(\xi \mid \zeta)_{\mathcal{X}}+\left(\left(A_{N}+\sigma\right) \xi \mid \Pi \zeta\right)_{\mathcal{X}}+\left(\Pi \xi \mid\left(A_{N}+\sigma\right) \zeta\right)_{\mathcal{X}}-\sum_{j=1}^{K}\left(B^{*} \Pi \xi \mid v_{j}\right) \mathcal{V}\left(B^{*} \Pi \zeta \mid v_{j}\right) \mathcal{V} & =0 \\
(\Pi \xi \mid \zeta)_{\mathcal{X}}=(\xi \mid \Pi \zeta)_{\mathcal{X}} \quad \text { and } \quad(\Pi \xi \mid \xi)_{\mathcal{X}}>0 \text { if } \xi & \neq 0 .
\end{aligned}
$$

The uniqueness of the solution of the above Riccati equation follows by remarking that every $\Pi \in \mathcal{L}\left(\mathcal{X}_{N}, \mathcal{X}_{N}^{*}\right)$ with orthogonal projection $\widetilde{\Pi}$ on $\mathcal{X}_{N}$ necessarily obeys $\Pi=P_{N}^{*} \widetilde{\Pi}$. Thus, because we have $V_{N}^{*} \widetilde{\Pi}=V^{*} \Pi$, the optimal pair obeys $\bar{u}_{\sigma}(t)=-V^{*} \Pi y_{N, \sigma}(t)$ and then the optimal state is solution to:

$$
y_{N, \sigma}^{\prime}=\left(A_{N}+\sigma\right) y_{N, \sigma}-V_{N}\left(V^{*} \Pi\right) y_{N, \sigma}, \quad y_{N, \sigma}(0)=h .
$$


Moreover, we have the exponential decrease

$$
\left\|y_{N, \sigma}(t)\right\|_{\mathcal{X}_{N}} \leq C e^{-a t}\|h\|_{\mathcal{X}_{N}} \quad a>0 .
$$

Thus, with the change of variable $\left(y_{N}(t), \bar{u}(t)\right)=e^{-\sigma t}\left(y_{N, \sigma}(t), \bar{u}_{\sigma}(t)\right)$ we obtain that the solution to:

$$
y_{N}^{\prime}=A_{N} y_{N}-V_{N}\left(V^{*} \Pi\right) y_{N}, \quad y_{N}(0)=h,
$$

obeys the exponential decrease:

$$
\left\|y_{N}(t)\right\|_{\mathcal{X}_{N}} \leq C e^{-(\sigma+a) t}\|h\|_{\mathcal{X}_{N}} \quad a>0
$$

and the feedback relation $\left(u_{1}, \ldots, u_{K}\right)=\bar{u}=-V^{*} \Pi y_{N}$ ensures that the optimal control $u=\sum_{j=1}^{K} u_{j} v_{j}$ obeys:

$$
\|u(t)\|_{\mathcal{V}} \leq C e^{-(\sigma+a) t} \quad a>0 .
$$

Finally by recalling the expression of $V^{*}$ given in (23) we deduce that $u$ is expressed as follows:

$$
u(t)=-\sum_{j=1}^{K}\left(v_{j} \mid B^{*} \Pi y_{N}(t)\right)_{\mathcal{V}} v_{j}, \quad t \geq 0 .
$$

\subsection{Dynamical stabilization of real finite-dimensional projected system}

Let us assume that Conditions 1 and 2 of Corollary 4 hold and let us consider the real family $\left(v_{j}\right)_{1 \leq j \leq K}$ given by Theorem 7. The main goal of this subsection is to prove that for $\sigma>0$ satisfying (10), system (34) is stabilizable with a rate of decrease $-\sigma$, by means of $u=\sum_{j=1}^{K} u_{j} v_{j}$ where $\bar{u}=\left(u_{1}, \ldots, u_{K}\right)$ is solution to:

$$
\bar{u}^{\prime}=D_{K} \bar{u}+G_{K} \bar{g},
$$

and where $D_{K} \in \mathcal{L}\left(\mathbb{R}^{K}\right), G_{K} \in \mathcal{L}\left(\mathbb{R}^{K}\right)$ and $\bar{g} \in\left(L^{2}(\mathbb{R})\right)^{K}$. Here $\bar{g}$ is the control of (34), (44). By setting $Y_{N}={ }^{t}\left(y_{N}, \bar{u}\right)$, system $(34),(44)$ can be rewritten as:

$$
Y_{N}^{\prime}=\mathbb{A}_{N} Y_{N}+\mathbb{B}_{N} \bar{g} \quad \text { where } \quad \mathbb{A}_{N} \stackrel{\text { def }}{=}\left(\begin{array}{cc}
A_{N} & V_{N} \\
0 & D_{K}
\end{array}\right) \quad \text { and } \quad \mathbb{B}_{N} \stackrel{\text { def }}{=}\left(\begin{array}{c}
0 \\
G_{K}
\end{array}\right) .
$$

The following theorem states that if $G_{K}$ is invertible then (45) is exactly controllable.

Theorem 8. Suppose that Conditions 1 and 2 of Corollary 4 hold and suppose that $G_{K} \in \mathcal{L}\left(\mathbb{R}^{K}\right)$ is invertible. Then (45) is exactly controllable.

Proof. Let denote $\Sigma_{K}$ the spectrum of $D_{K}$. Since $\Sigma_{K} \cup \Sigma_{N}$ is exactly the spectrum of $\mathbb{A}_{N}$, the controllability of (45) follows from the following Hautus rank condition:

$$
\operatorname{Rank}\left(\begin{array}{cc|c}
A_{N}-\lambda & V_{N} & 0 \\
0 & D_{K}-\lambda & G_{K}
\end{array}\right)=\operatorname{dim}\left(\mathcal{X}_{N}\right)+K \quad \forall \lambda \in \Sigma_{K} \cup \Sigma_{N},
$$

which is an easy consequence of the assumptions. Indeed, the above equality is equivalent to the fact that for all $\lambda \in \Sigma_{K} \cup \Sigma_{N}$ and $(f, \bar{h}) \in \mathcal{X}_{N} \times \mathbb{R}^{K}$ the system

$$
\begin{aligned}
\left(A_{N}-\lambda\right) e+V_{N} \bar{v} & =z, \\
\left(D_{K}-\lambda\right) \bar{v}+G_{K} \bar{g} & =\bar{h},
\end{aligned}
$$

admits a solution $(e, \bar{v}, \bar{g}) \in \mathcal{X}_{N} \times \mathbb{R}^{K} \times \mathbb{R}^{K}$. Since Conditions 1 and 2 of Corollary 4 guarantee the controllability of (34), and then imply the Hautus test, $\operatorname{Rank}\left(A_{N}-\lambda \mid V_{N}\right)=\operatorname{dim}\left(\mathcal{X}_{N}\right)$ for all $\lambda \in \Sigma_{N}$ (and then obviously for $\lambda \in \Sigma_{K} \cup \Sigma_{N}$ also), then there exists a pair $(e, \bar{v}) \in \mathcal{X}_{N} \times \mathbb{R}^{K}$ satisfying the first equation and it suffices to choose $\bar{g}=G_{K}^{-1}\left(\bar{h}-\left(D_{K}-\lambda\right) \bar{v}\right)$.

Remark 9. Although an obvious choice for $\left(D_{K}, G_{K}\right)$ is $D_{K}=0$ and $G_{K}=I_{\mathbb{R}^{K}}$ (identity in $\mathbb{R}^{K}$ ), in the following we choose to consider the general case $\left(D_{K}, G_{K}\right) \in \mathcal{L}\left(\mathbb{R}^{K}\right) \times \mathcal{L}\left(\mathbb{R}^{K}\right)$ with $G_{K}$ invertible. Indeed, the efficiency of the stabilizing control which is constructed below could be related to the choice of $\left(D_{K}, G_{K}\right)$. For instance, a challenging problem should be to determine how the constant $K_{s}$ of Theorem 18 below depends on such a choice. 
We suppose that the hypotheses of Theorem 8 are verified and we consider the system

$$
Y_{N, \sigma}^{\prime}=\left(\mathbb{A}_{N}+\sigma\right) Y_{N, \sigma}+\mathbb{B}_{N} \bar{g}_{\sigma}
$$

which is also exactly controllable. For $H \in \mathcal{X}_{N} \times \mathbb{R}^{K}$ we define the following optimal control problem:

$$
\inf \left\{\frac{1}{2} \int_{0}^{\infty}\left\|Y_{N, \sigma}\right\|_{\mathcal{X}_{N} \times \mathbb{R}^{K}}^{2} d t+\frac{1}{2} \int_{0}^{\infty}\left\|\bar{g}_{\sigma}(t)\right\|_{\mathbb{R}^{K}}^{2} d t ;\left(Y_{N, \sigma}, \bar{g}_{\sigma}\right) \text { obeys }(46) \text { and } Y_{N, \sigma}(0)=H\right\} .
$$

According to Theorem 2.9 p. 44, Theorem 1.4 p. 136 and Theorem 1.5 p. 138 in [41] the above minimization problem admits a unique solution $\left(Y_{N, \sigma}, \bar{g}_{\sigma}\right)$ which is exponentially stable:

$$
\left\|Y_{N, \sigma}(t)\right\|_{\mathcal{X} \times \mathbb{R}^{K}}+\left\|\bar{g}_{\sigma}(t)\right\|_{\mathbb{R}^{K}} \leq C e^{-a t}\|H\|_{\mathcal{X} \times \mathbb{R}^{K}}, \quad a>0,
$$

and which obeys $\bar{g}_{\sigma}(t)=-\mathbb{B}_{N}^{*} \Pi Y_{N, \sigma}(t)$ where $\boldsymbol{\Pi} \in \mathcal{L}\left(\mathcal{X}_{N} \times \mathbb{R}^{K}\right)$ is the unique solution of the Riccati equation: for all $(\xi, \zeta) \in\left(\mathcal{X}_{N} \times \mathbb{R}^{K}\right) \times\left(\mathcal{X}_{N} \times \mathbb{R}^{K}\right)$

$$
\begin{aligned}
&(\xi \mid \zeta)_{\mathcal{X} \times \mathbb{R}^{K}}+\left(\left(\mathbb{A}_{N}+\sigma\right) \xi \mid \boldsymbol{\Pi} \zeta\right)_{\mathcal{X} \times \mathbb{R}^{K}}+\left(\boldsymbol{\Pi} \xi \mid\left(\mathbb{A}_{N}+\sigma\right) \zeta\right)_{\mathcal{X} \times \mathbb{R}^{K}}-\left(\mathbb{B}_{N}^{*} \boldsymbol{\Pi} \xi \mid \mathbb{B}_{N}^{*} \boldsymbol{\Pi} \zeta\right)_{\mathcal{X} \times \mathbb{R}^{K}}=0 \\
&(\boldsymbol{\Pi} \xi \mid \zeta)_{\mathcal{X} \times \mathbb{R}^{K}}=(\xi \mid \boldsymbol{\Pi} \zeta)_{\mathcal{X} \times \mathbb{R}^{K}} \quad \text { and }(\boldsymbol{\Pi} \xi \mid \xi)_{\mathcal{X} \times \mathbb{R}^{K}>0 \text { if } \xi} \neq 0
\end{aligned}
$$

Then from (46) with $\bar{g}_{\sigma}(t)=-\mathbb{B}_{N}^{*} \Pi Y_{N, \sigma}(t)$ and from (48), we obtain that the pair $\left(Y_{N}, \bar{g}\right)=e^{-\sigma t}\left(Y_{N, \sigma}, \bar{g}_{\sigma}\right)$ obeys

$$
Y_{N}^{\prime}=\mathbb{A}_{N} Y_{N}-\mathbb{B}_{N} \mathbb{B}_{N}^{*} \Pi Y_{N}, \quad Y_{N}(0)=H, \quad \bar{g}(t)=-\mathbb{B}_{N}^{*} \Pi Y_{N}(t),
$$

and

$$
\left\|Y_{N}(t)\right\|_{\mathcal{X} \times \mathbb{R}^{K}}+\|\bar{g}(t)\|_{\mathbb{R}^{K}} \leq C e^{-(\sigma+a) t}\|H\|_{\mathcal{X} \times \mathbb{R}^{K}}, \quad a>0 .
$$

Thus, by recalling the expression of $\mathbb{A}_{N}$ and $\mathbb{B}_{N}$ given in (45), and by writing

$$
\boldsymbol{\Pi}=\left(\begin{array}{ll}
\Pi_{1} & \Pi_{2}^{*} \\
\Pi_{2} & \Pi_{3}
\end{array}\right) \quad \text { with } \quad \Pi_{1} \in \mathcal{L}\left(\mathcal{X}_{N}\right), \quad \Pi_{2} \in \mathcal{L}\left(\mathcal{X}_{N}, \mathbb{R}^{K}\right) \quad \text { and } \quad \Pi_{3} \in \mathcal{L}\left(\mathbb{R}^{K}\right)
$$

we deduce that the closed-loop system satisfied by $Y_{N}={ }^{t}\left(y_{N}, \bar{u}\right)$ (with $\left.H={ }^{t}(h, \bar{\varsigma})\right)$ can be equivalently written as follows:

$$
\left\{\begin{aligned}
y_{N}^{\prime} & =A_{N} y_{N}+V_{N} \bar{u} & & y_{N}(0)=h \\
\bar{u}^{\prime} & =D_{K} \bar{u}-G_{K} G_{K}^{*} \Pi_{2} y_{N}-G_{K} G_{K}^{*} \Pi_{3} \bar{u}, & & \bar{u}(0)=\bar{\varsigma}
\end{aligned}\right.
$$

and that its solution $\left(y_{N}, \bar{u}\right)$ obeys:

$$
\left\|y_{N}(t)\right\|_{\mathcal{X}}+\|\bar{u}(t)\|_{\mathbb{R}^{K}} \leq C e^{-(\sigma+a) t}\left(\|h\|_{\mathcal{X}}+\|\bar{\zeta}\|_{\mathbb{R}^{K}}\right) .
$$

\section{Stabilization of the infinite-dimensional system}

Here, we make some additional assumptions on $A$ and $B$. Set $\widehat{A} \stackrel{\text { def }}{=} \lambda_{0}-A$ for $\lambda_{0}>0$ large enough so that the fractional powers of $\widehat{A}$ are well-defined, suppose that $B$ is strictly relatively bounded with respect to $A$ :

$$
\widehat{A}^{-\gamma} B: \mathcal{V} \rightarrow \mathcal{X} \quad \text { is bounded for some } 0 \leq \gamma<1,
$$

that the following complex interpolation equalities hold:

$$
\mathcal{D}\left(\widehat{A}^{\theta}\right)=[\mathcal{D}(A), \mathcal{X}]_{1-\theta} \quad \text { and } \quad \mathcal{D}\left(\widehat{A}^{* \theta}\right)=\left[\mathcal{D}\left(A^{*}\right), \mathcal{X}\right]_{1-\theta} \quad \forall \theta \in[0,1],
$$

and that there is a Hilbert space $\mathcal{O}$ and a bounded operator $G \in \mathcal{L}(\mathcal{X}, \mathcal{O})$ such that:

$$
G \text { admits a bounded inverse and } G^{*} G \in \mathcal{L}\left(\mathcal{D}\left(\widehat{A}^{\frac{1}{2}}\right), \mathcal{D}\left(\widehat{A}^{* \frac{1}{2}}\right)\right) \text {. }
$$

For a family $\left(v_{j}\right)_{j=1, \ldots, K}$ given by Theorem 7 which is such that (34) is exactly controllable, we consider system (9) with a finite dimensional control $u=\sum_{j=1}^{K} u_{j} v_{j}$ for $\bar{u}=\left(u_{1}, \ldots, u_{K}\right) \in L^{2}\left(\mathbb{R}^{K}\right)$ and for an initial datum $y_{0} \in \mathcal{X}$. With the notations of Subsection 3.2 such a system can be rewritten as follows:

$$
y^{\prime}=A y+V \bar{u} \in\left[\mathcal{D}\left(A^{*}\right)\right]^{\prime}, \quad y(0)=y_{0} .
$$


We will prove the stability of (56) when $\bar{u}$ is the finite dimensional feedback control obtained in Subsection 3.4 or when $\bar{u}$ is the finite dimensional dynamical control obtained in Subsection 3.5, see Propositions 10 and 17 below. Moreover, for the same types of controls, we will also prove the local stability of the nonlinear system:

$$
y^{\prime}=A y+V \bar{u}+F(y, \bar{u}) \in\left[\mathcal{D}\left(A^{*}\right)\right]^{\prime}, \quad y(0)=y_{0},
$$

under suitable boundedness and Lipschitz assumptions on the nonlinear mapping $F$ (see (92), (95)), see Theorems 15 and 18 below. Notice that we consider a nonlinearity $F(y, \bar{u})$ depending on both variables $y$ and $\bar{u}$ in order to cover the case of Navier-Stokes equations with Dirichlet control. In that case, the velocity of the fluid contains a part depending on $\bar{u}$ (see the decomposition (111)-(112) and the equation (113)).

\subsection{Feedback stabilization with finite-dimensional controllers}

Let us consider system (56) with the feedback control $\bar{u}(t)=-V^{*} \Pi P_{N} y(t)$ obtained in Subsection 3.4:

$$
y^{\prime}=A y-V V^{*} \Pi P_{N} y \in\left[\mathcal{D}\left(A^{*}\right)\right]^{\prime}, \quad y(0)=y_{0} .
$$

Notice that the above system corresponds to system (9) with the finite dimensional feedback control given by

$$
u(t)=-\sum_{j=1}^{K}\left(v_{j} \mid B^{*} \Pi P_{N} y(t)\right) \mathcal{v} v_{j}
$$

Proposition 10. For $y_{0} \in \mathcal{X},(57)$ admits a unique solution given by $y(t)=e^{-A_{\Pi} t} y_{0}$, where $\left(e^{-A_{\Pi} t}\right)_{t \geq 0}$ is an analytic semigroup on $\mathcal{X}$ with infinitesimal generator $-A_{\Pi}$ defined by

$$
\mathcal{D}\left(A_{\Pi}\right) \stackrel{\text { def }}{=}\left\{\xi \in \mathcal{X} \mid A \xi-V V^{*} \Pi P_{N} \xi \in \mathcal{X}\right\} \quad \text { and } \quad A_{\Pi} \xi \stackrel{\text { def }}{=} V V^{*} \Pi P_{N} \xi-A \xi
$$

The semigroup $\left(e^{-A_{\Pi} t}\right)_{t \geq 0}$ is exponentially stable with an exponential rate strictly lower than $-\sigma$ :

$$
\inf \left\{\Re \lambda \mid \lambda \in \sigma\left(A_{\Pi}\right)\right\}>\sigma .
$$

The adjoint of $A_{\Pi}$ is given by:

$$
\mathcal{D}\left(A_{\Pi}^{*}\right)=\mathcal{D}\left(A^{*}\right) \quad \text { and } \quad A_{\Pi}^{*} \xi=P_{N}^{*}\left(V^{*} \Pi\right)^{*} V^{*} \xi-A^{*} \xi
$$

Proof. Definition (59) means that $\xi \in \mathcal{D}\left(A_{\Pi}\right)$ if and only if $\zeta \in \mathcal{D}\left(A^{*}\right) \mapsto\left\langle A \xi-V V^{*} \Pi P_{N} \xi \mid \zeta\right\rangle_{\left[\mathcal{D}\left(A^{*}\right)\right]^{\prime}, \mathcal{D}\left(A^{*}\right)}$ is bounded for the $\mathcal{X}$-topology, and since $\left\langle A \xi-V V^{*} \Pi P_{N} \xi \mid \zeta\right\rangle_{\left[\mathcal{D}\left(A^{*}\right)\right]^{\prime}, \mathcal{D}\left(A^{*}\right)}=\left(\xi \mid P_{N}^{*}\left(V^{*} \Pi\right)^{*} V^{*} \zeta-A^{*} \zeta\right)_{\mathcal{X}}$ it exactly means that $\left(\mathcal{D}\left(A_{\Pi}\right), A_{\Pi}\right)$ is the adjoint of $\left(\mathcal{D}\left(A^{*}\right), P_{N}^{*}\left(V^{*} \Pi\right)^{*} V^{*}-A^{*}\right)$. Then $(61)$ is proved. Thus, since $P_{N}^{*}\left(V^{*} \Pi\right)^{*} V^{*}$ belongs to $\mathcal{L}\left(\mathcal{D}\left(\widehat{A}^{* \gamma}\right), \mathcal{X}\right)$, a perturbation argument ensures that $\left(\mathcal{D}\left(A_{\Pi}^{*}\right), A_{\Pi}^{*}\right)$ is the infinitesimal generator of an analytic semigroup on $\mathcal{X}$ [31, Chap. 3, Cor. 2.4], and the analyticity of $A_{\Pi}$ follows from a duality argument. As a consequence, if $y_{0} \in \mathcal{X}$ then $y(t)=e^{-A_{\Pi} t} y_{0}$ obeys $y^{\prime}=-A_{\Pi} y \in\left[\mathcal{D}\left(A_{\Pi}^{*}\right)\right]^{\prime}$ and $y(0)=y_{0}$ i.e. $y$ is the solution of (57). Let us now verify that $\left(e^{-A_{\Pi} t}\right)_{t \geq 0}$ is also exponentially stable. Since system (57) corresponds to (9) with (58), by following the steps of Subsection 3.3 (see (31) and (32)) it can be rewritten as:

$$
\begin{aligned}
y_{N}^{\prime} & =A_{N} y_{N}+B_{N} u \in \mathcal{X}_{N}, \quad y_{N}(0)=P_{N} y_{0} \\
y_{N}^{-\prime^{\prime}} & =A_{N}^{-} y_{N}^{-}+B_{N}^{-} p_{N}^{-} u \in\left[\mathcal{D}\left(A^{*}\right) \cap \mathcal{X}_{N}^{*-}\right]^{\prime}, \quad y_{N}(0)=\left(I-P_{N}\right) y_{0},
\end{aligned}
$$

where $\left(y_{N}, y_{N}^{-}\right)=\left(P_{N} y,\left(I-P_{N}\right) y\right)$ and $u$ is given by (58). Moreover, because the first above equality can be equivalently rewritten as (40) with $h=P_{N} y_{0}$, then from (41) we already know that $y_{N}$ is exponentially stable with a rate of decrease $-(\sigma+a)$, and to obtain $(60)$ it remains to prove the exponential decay of $y_{N}^{-}(t)$ with a rate $-(\sigma+a)$. We start by using the Duhamel representation:

$$
y_{N}^{-}(t)=e^{A_{N}^{-} t}\left(I-P_{N}\right) y_{0}-\int_{0}^{t} e^{A_{N}^{-}(t-\tau)} B_{N}^{-} p_{N}^{-} u(\tau) d \tau .
$$

According to [9, Part. II, Chap. 1, Cor. 2.1 p. 92 and Prop. 2.9, p.120], the fact that $\Re \lambda_{N+1}=\sup \{\Re \lambda \mid$ $\left.\lambda \in \sigma\left(A_{N}^{-}\right)\right\}<-\sigma-\epsilon<0$ for some $\epsilon>0$ combined with the analyticity of $\left(e^{A_{N}^{-} t}\right)_{t \geq 0}$ on $\mathcal{X}_{N}^{-}$implies that the exponential decrease of $\left(e^{A_{N}^{-}}\right)_{t \geq 0}$ has a rate $-\sigma-\epsilon$. Moreover, we set $\widehat{A}_{N}^{-}=\lambda_{0}-A_{N}^{-}$, we remark that we have 
$\left(\widehat{A}_{N}^{-}\right)^{-\gamma}=\left(I-P_{N}\right) \widehat{A}^{-\gamma}$ and $B_{N}^{-}=\left(I-P_{N}\right) B=\left(\widehat{A}_{N}^{-}\right)^{\gamma}\left(I-P_{N}\right) \widehat{A}^{-\gamma} B$, and the analyticity of $\left(e^{A_{N}^{-} t}\right)_{t \geq 0}$ with (53) gives:

$$
\left\|e^{A_{N}^{-} t} B_{N}^{-} u\right\|_{\mathcal{X}}=\left\|\left(\widehat{A}_{N}^{-}\right)^{\gamma} e^{A_{N}^{-} t}\left(I-P_{N}\right) \widehat{A}^{-\gamma} B u\right\|_{\mathcal{X}} \leq C \frac{e^{-(\sigma+\epsilon) t}}{t^{\gamma}}\|u\|_{\mathcal{V}}, \quad \forall t>0 .
$$

The above estimate combined with (62) and (42) yields:

$$
\begin{aligned}
\left\|y_{N}^{-}(t)\right\|_{\mathcal{X}} & \leq C e^{-(\sigma+\epsilon) t}\left\|\left(I-P_{N}\right) y_{0}\right\|_{\mathcal{X}}+C \int_{0}^{t} \frac{e^{-(\sigma+\epsilon)(t-\tau)}}{(t-\tau)^{\gamma}} e^{-(\sigma+a) \tau}\left\|P_{N} y_{0}\right\|_{\mathcal{X}} d \tau, \\
& \leq C\left(e^{-(\sigma+\epsilon) t}+e^{-(\sigma+a) t} \int_{0}^{t} \frac{e^{(a-\epsilon)(t-\tau)}}{(t-\tau)^{\gamma}} d \tau\right)\left\|y_{0}\right\|_{\mathcal{X}}
\end{aligned}
$$

and thus up to decreasing $a$ so that $a<\epsilon$ we obtain that $\left\|y_{N}^{-}(t)\right\|_{\mathcal{X}} \leq C\left\|y_{0}\right\|_{\mathcal{X}} e^{-(\sigma+a) t}$.

Remark 11. Notice that assumption (54) is not used in the proof of Proposition 10.

Let us now explain how to construct a Lyapunov function for the closed-loop system (57). First, since $-A_{\Pi}$ generates an analytic and exponentially stable semigroup on $\mathcal{X}$, it is boundedly invertible [9, Part. II, Chap. 1, Prop. 2.9, p. 120], the fractional powers of $A_{\Pi}$ are well-defined [9, Part II, Chap. 1, Par. 5 p. 167] and (54) combined with a perturbation argument [17, Prop. 2.7] ensures that the complex interpolation equalities $\mathcal{D}\left(A_{\Pi}^{\theta}\right)=\left[\mathcal{D}\left(A_{\Pi}\right), \mathcal{X}\right]_{1-\theta}$ and $\mathcal{D}\left(A_{\Pi}^{* \theta}\right)=\left[\mathcal{D}\left(A_{\Pi}^{*}\right), \mathcal{X}\right]_{1-\theta}$ hold for all $\theta \in[0,1]$. Then for $\theta \in[0,1]$ we introduce the spaces:

$$
\mathcal{X}^{2 \theta} \stackrel{\text { def }}{=} \mathcal{D}\left(A_{\Pi}^{\theta}\right), \quad \mathcal{X}_{*}^{2 \theta} \stackrel{\text { def }}{=} \mathcal{D}\left(A_{\Pi}^{* \theta}\right), \quad \mathcal{X}^{-2 \theta} \stackrel{\text { def }}{=}\left[\mathcal{X}_{*}^{2 \theta}\right]^{\prime} \text { and } \mathcal{X}_{*}^{-2 \theta} \stackrel{\text { def }}{=}\left[\mathcal{X}^{2 \theta}\right]^{\prime},
$$

respectively normed with:

$$
\|\cdot\|_{\mathcal{X}^{2 \theta}} \stackrel{\text { def }}{=}\left\|A_{\Pi}^{\theta} \cdot\right\|_{\mathcal{X}}, \quad\|\cdot\|_{\mathcal{X}_{*}^{2 \theta}} \stackrel{\text { def }}{=}\left\|A_{\Pi}^{* \theta} \cdot\right\|_{\mathcal{X}}, \quad\|\cdot\|_{\mathcal{X}^{-2 \theta}} \stackrel{\text { def }}{=}\left\|A_{\Pi}^{-\theta} \cdot\right\|_{\mathcal{X}} \quad \text { and } \quad\|\cdot\|_{\mathcal{X}_{*}^{-2 \theta}} \stackrel{\text { def }}{=}\left\|A_{\Pi}^{*-\theta} \cdot\right\|_{\mathcal{X}} .
$$

Notice that because $A_{\Pi}^{-\theta}=\left(A_{\Pi}^{\theta}\right)^{-1}$ and $A_{\Pi}^{*-\theta}=\left(A_{\Pi}^{* \theta}\right)^{-1}$, the above dual norm's definition can be obtained from [39, Chap. 2, Prop. 2.10.2]. We introduce the following linear and bounded operator:

$$
T \stackrel{\text { def }}{=} I+\widehat{A}^{-1} V\left(V^{*} \Pi\right) P_{N}: \mathcal{X} \rightarrow \mathcal{X}
$$

and the next proposition gives a characterization of the spaces $\mathcal{X}^{2 \theta}$ and $\mathcal{X}_{*}^{2 \theta}$ by using $T$.

Proposition 12. Let $\theta \in[0,1]$. Then the following results hold:

1. $T$ is an isomorphism from $\mathcal{D}\left(A_{\Pi}^{\theta}\right)$ onto $\mathcal{D}\left(\widehat{A}^{\theta}\right)$.

2. $T^{*}$ is an isomorphism from $\mathcal{D}\left(\widehat{A}^{* \theta}\right)$ onto itself.

3. The following characterizations hold:

$$
\mathcal{D}\left(A_{\Pi}^{\theta}\right)=\left\{\xi \in \mathcal{X} \mid T \xi \in \mathcal{D}\left(\widehat{A}^{\theta}\right)\right\} \quad \text { and } \quad \mathcal{D}\left(A_{\Pi}^{* \theta}\right)=\mathcal{D}\left(\widehat{A}^{* \theta}\right) .
$$

Proof. First, since we obviously have $T \in \mathcal{L}(\mathcal{X})$, and since from (59) we deduce that:

$$
\mathcal{D}\left(A_{\Pi}\right)=\left\{\xi \in \mathcal{X} \mid \xi+\widehat{A}^{-1} V\left(V^{*} \Pi\right) P_{N} \xi \in \mathcal{D}(A)\right\}=\{\xi \in \mathcal{X} \mid T \xi \in \mathcal{D}(A)\}
$$

which also means that $T \in \mathcal{L}\left(\mathcal{D}\left(A_{\Pi}\right), \mathcal{D}(A)\right)$, then $T \in \mathcal{L}\left(\left[\mathcal{D}\left(A_{\Pi}\right), \mathcal{X}\right]_{1-\theta},[\mathcal{D}(A), \mathcal{X}]_{1-\theta}\right)=\mathcal{L}\left(\mathcal{D}\left(A_{\Pi}^{\theta}\right), \mathcal{D}\left(\widehat{A}^{\theta}\right)\right)$ follows by interpolation. Next, to prove that $T: \mathcal{X} \rightarrow \mathcal{X}$ is boundedly invertible we first remark that it is a compact perturbation of the identity, and then that it suffices to prove its injectivity. Let us suppose that $\xi \in \mathcal{X}$ obeys the equality $T \xi=\xi+\widehat{A}^{-1} V\left(V^{*} \Pi\right) P_{N} \xi=0$. By multiplying by $\widehat{A}^{*} \Pi P_{N} \xi \in \mathcal{X}_{N}^{*}$ we obtain $\lambda_{0}\left(P_{N} \xi \mid \Pi P_{N} \xi\right)_{\mathcal{X}}-\left(A_{N} P_{N} \xi \mid \Pi P_{N} \xi\right)_{\mathcal{X}}+\left\|\left(V^{*} \Pi\right) P_{N} \xi\right\|_{\mathbb{R}^{K}}^{2}=0$ and, with (36) applied to $\left(P_{N} \xi, P_{N} \xi\right)$, we obtain:

$$
\left(\lambda_{0}+\sigma\right)\left(P_{N} \xi \mid \Pi P_{N} \xi\right)_{\mathcal{X}}+\frac{1}{2}\left\|P_{N} \xi\right\|_{\mathcal{X}}^{2}+\frac{1}{2} \sum_{j=1}^{K}\left(B^{*} \Pi P_{N} \xi \mid v_{j}\right)_{\mathcal{V}}^{2}=0
$$

which guarantees that $P_{N} \xi=0$. Thus, since $\xi+\widehat{A}^{-1} V\left(V^{*} \Pi\right) P_{N} \xi=0$ we finally deduce that $\xi=0$. Then we have proved that $T$ is an isomorphism from $\mathcal{X}$ onto $\mathcal{X}$. Moreover, since (66) exactly means that $T^{-1}$ maps $\mathcal{D}(A)$ to $\mathcal{D}\left(A_{\Pi}\right)$, we obtain $T^{-1} \in \mathcal{L}\left([\mathcal{D}(A), \mathcal{X}]_{1-\theta},\left[\mathcal{D}\left(A_{\Pi}\right), \mathcal{X}\right]_{1-\theta}\right)=\mathcal{L}\left(\mathcal{D}\left(\widehat{A}^{\theta}\right), \mathcal{D}\left(A_{\Pi}^{\theta}\right)\right)$ by interpolation. 
Next, we have $T^{*}=I+P_{N}^{*}\left(V^{*} \Pi\right)^{*} V^{*} \widehat{A}^{*-1}$ and since $P_{N}^{*} \in \mathcal{L}\left(\mathcal{X}, \mathcal{D}\left(A^{*}\right)\right)$ we deduce that $T^{*} \in \mathcal{L}\left(\mathcal{D}\left(\widehat{A}^{* \theta}\right)\right)$. Moreover, since $T: \mathcal{X} \rightarrow \mathcal{X}$ is boundedly invertible then so does $T^{*}$, and we obtain that $T^{*-1} \in \mathcal{L}\left(\mathcal{D}\left(\widehat{A}^{* \theta}\right)\right)$ from $P_{N}^{*} \in \mathcal{L}\left(\mathcal{X}, \mathcal{D}\left(A^{*}\right)\right)$ by remarking that $T^{*-1}=I-P_{N}^{*}\left(V^{*} \Pi\right)^{*} V^{*} \widehat{A}^{*-1} T^{*-1}$. Finally, the second equality in $(65)$ is a direct consequence of $(61)$

An obvious consequence of the above proposition and of (55) is the following corollary:

Corollary 13. The operator $T^{*} G^{*} G T$ is an isomorphism from $\mathcal{X}$ onto itself as well as from $\mathcal{X}^{1}$ onto $\mathcal{X}_{*}^{1}$.

By following an approach which is inspired from [30], we introduce a linear operator $L_{s}$ depending on $A_{\Pi}$ and on $T^{*} G^{*} G T$ which will define a Lyapunov function for system (57):

Proposition 14. Let $T$ be given by (64) and $G \in \mathcal{L}(\mathcal{X}, \mathcal{O})$ be an operator obeying (55). Then the operator

$$
L_{s} \stackrel{\text { def }}{=} \int_{0}^{\infty} e^{2 \sigma t} e^{-A_{\Pi}^{*} t} A_{\Pi}^{* \frac{1}{2}+\frac{s}{2}} T^{*} G^{*} G T A_{\Pi}^{\frac{1}{2}+\frac{s}{2}} e^{-A_{\Pi} t} d t
$$

is bounded from $\mathcal{X}^{s}$ to $\mathcal{X}_{*}^{-s}$ as well as from $\mathcal{X}^{1+s}$ to $\mathcal{X}_{*}^{1-s}$. Moreover, the following equalities hold

$$
\begin{aligned}
\left\langle L_{s} \xi \mid \xi\right\rangle_{\mathcal{X}_{*}^{-s}, \mathcal{X}^{s}} & =\int_{0}^{\infty} e^{2 \sigma t}\left\|G T A_{\Pi}^{\frac{1}{2}+\frac{s}{2}} e^{-A_{\Pi} t} \xi\right\|_{\mathcal{O}}^{2} d t \quad \forall \xi \in \mathcal{X}^{s}, \\
\left\langle L_{s} \xi \mid A_{\Pi} \xi\right\rangle_{\mathcal{X}_{*}^{1-s}, \mathcal{X}^{s-1}} & =\frac{1}{2}\left\|G T A_{\Pi}^{\frac{1}{2}+\frac{s}{2}} \xi\right\|_{\mathcal{O}}^{2}+\sigma\left\langle L_{s} \xi \mid \xi\right\rangle_{\mathcal{X}_{*}^{-s}, \mathcal{X}^{s}} \quad \forall \xi \in \mathcal{X}^{s+1},
\end{aligned}
$$

and define norms on $\mathcal{X}^{s}$ and $\mathcal{X}^{s+1}$ respectively. These norms are equivalent to the norms defined by (63).

In the following, we will use the notations:

$$
\|\| \cdot\|\|_{s} \stackrel{\text { def }}{=}\left(\left\langle L_{s} \cdot \mid \cdot\right\rangle_{\mathcal{X}_{*}^{-s}, \mathcal{X}^{s}}\right)^{1 / 2} \quad \text { and } \quad \mathcal{N}_{s+1}(\cdot) \stackrel{\text { def }}{=}\left\|G T A_{\Pi}^{\frac{1}{2}+\frac{s}{2}} \cdot\right\|_{\mathcal{O}}
$$

Proof. First, since $y=e^{-\left(A_{\Pi}-\sigma\right)(\cdot)} \xi$ is the solution of $y^{\prime}+\left(A_{\Pi}-\sigma\right) y=0$ and $y(0)=\xi$, from maximal regularity results for analytic semigroup (8) we deduce that

$$
A_{\Pi}^{\frac{1}{2}+\frac{s}{2}} e^{-\left(A_{\Pi}-\sigma\right)(\cdot)} \in \mathcal{L}\left(\mathcal{X}^{s}, L^{2}(\mathcal{X})\right) \cap \mathcal{L}\left(\mathcal{X}^{s+1}, L^{2}\left(\mathcal{X}^{1}\right)\right),
$$

and by using Corollary 13 we obtain:

$$
T^{*} G^{*} G T A_{\Pi}^{\frac{1}{2}+\frac{s}{2}} e^{-\left(A_{\Pi}-\sigma\right)(\cdot)} \in \mathcal{L}\left(\mathcal{X}^{s}, L^{2}(\mathcal{X})\right) \cap \mathcal{L}\left(\mathcal{X}^{s+1}, L^{2}\left(\mathcal{X}_{*}^{1}\right)\right) .
$$

Next, let us introduce $\mathcal{K} \in \mathcal{L}\left(L^{2}(\mathcal{X})\right)$ as follows:

$$
\mathcal{K}(f)(t)=\int_{0}^{t} e^{-\left(A_{\Pi}-\sigma\right)(t-\tau)} f(\tau) d \tau .
$$

An easy calculation shows that the adjoint of $\mathcal{K}$ with respect to $L^{2}(\mathcal{X})$ is given by

$$
\mathcal{K}^{*}(f)(t)=\int_{t}^{\infty} e^{-\left(A_{\Pi}^{*}-\sigma\right)(\tau-t)} f(\tau) d \tau
$$

Moreover, since $y=\mathcal{K}(f)$ is the solution of $y^{\prime}+\left(A_{\Pi}-\sigma\right) y=f$ and $y(0)=0$, maximal regularity results for analytic semigroup (8) apply and we have:

$$
\mathcal{K} \in \mathcal{L}\left(L^{2}\left(\mathcal{X}^{-2}\right), L^{2}(\mathcal{X})\right) \cap \mathcal{L}\left(L^{2}\left(\mathcal{X}^{-3}\right), L^{2}\left(\mathcal{X}^{-1}\right)\right) \quad \text { and } \quad \mathcal{K} A_{\Pi} \in \mathcal{L}\left(L^{2}(\mathcal{X})\right) \cap \mathcal{L}\left(L^{2}\left(\mathcal{X}^{-1}\right)\right) .
$$

Thus, a duality argument gives:

$$
\mathcal{K}^{*} \in \mathcal{L}\left(L^{2}(\mathcal{X}), L^{2}\left(\mathcal{X}_{*}^{2}\right)\right) \cap \mathcal{L}\left(L^{2}\left(\mathcal{X}_{*}^{1}, L^{2}\left(\mathcal{X}_{*}^{3}\right)\right)\right) \quad \text { and } \quad A_{\Pi}^{*} \mathcal{K}^{*} \in \mathcal{L}\left(L^{2}(\mathcal{X})\right) \cap \mathcal{L}\left(L^{2}\left(\mathcal{X}_{*}^{1}\right)\right),
$$

and since $\frac{d}{d t} \mathcal{K}^{*}=\left(A_{\Pi}^{*}-\sigma\right) \mathcal{K}^{*}-I$, we obtain:

$$
\mathcal{K}^{*} \in \mathcal{L}\left(L^{2}(\mathcal{X}), W\left(\mathcal{X}_{*}^{2}, \mathcal{X}\right)\right) \cap \mathcal{L}\left(L^{2}\left(\mathcal{X}_{*}^{1}\right), W\left(\mathcal{X}_{*}^{3}, \mathcal{X}_{*}^{1}\right)\right)
$$


Finally, with the continuous embeddings $W\left(\mathcal{X}_{*}^{2}, \mathcal{X}\right) \hookrightarrow C_{b}\left(\mathcal{X}_{*}^{1}\right)$ and $W\left(\mathcal{X}_{*}^{3}, \mathcal{X}_{*}^{1}\right) \hookrightarrow C_{b}\left(\mathcal{X}_{*}^{2}\right)$, and by remarking that $L_{s} \xi=A_{\Pi}^{* \frac{1}{2}+\frac{s}{2}} \mathcal{K}^{*}\left(T^{*} G^{*} G T A_{\Pi}^{\frac{1}{2}+\frac{s}{2}} e^{-\left(A_{\Pi}-\sigma\right)(\cdot)} \xi\right)(0)$, the first part of the proposition follows from (69) and (70).

Next, from (8) we deduce that square root of $\int_{0}^{\infty}\left\|A_{\Pi} e^{-\left(A_{\Pi}-\sigma\right) t} \cdot\right\|_{\mathcal{O}}^{2} d t$ defines a norm equivalent to $\|\cdot\|_{\mathcal{X}^{1}}$, and then so does the square root of $\int_{0}^{\infty}\left\|G T A_{\Pi} e^{-\left(A_{\Pi}-\sigma\right) t} \cdot\right\|_{\mathcal{O}}^{2} d t$ since $G T \in \mathcal{L}(\mathcal{X}, \mathcal{O})$ is boundedly invertible. Thus, by using the change of variable $\xi \mapsto A_{\Pi}^{\frac{s}{2}-\frac{1}{2}} \xi$ one obtains that (67) defines a norm equivalent to $\|\cdot\|_{\mathcal{X}^{s}}$. Finally, (68) follows from an integration by parts and the fact that it defines a norm on $\mathcal{X}^{s+1}$ follows from the bounded invertibility of $G T \in \mathcal{L}(\mathcal{X}, \mathcal{O})$.

If we now multiply the first equality in (57) by $L_{s} y$ we obtain:

$$
\frac{d}{d t}\||y(t)|\|_{s}^{2}+2 \sigma\left|\|y(t) \mid\|_{s}^{2}+\mathcal{N}_{s+1}(y(t))^{2}=0,\right.
$$

which means that $\left.|\| \cdot|\right|_{s} ^{2}$ is a Lyapunov function associated to $(57): t \mapsto\||| y(t) \mid\|_{s}^{2}$ decreases to zero and

$$
\|y(t)\|\left\|_{s} \leq e^{-\left(\sigma+\beta_{s}\right) t}\right\| y(0)\|\|_{s} \quad \text { where } \quad \beta_{s} \stackrel{\text { def }}{=} \inf _{0 \neq \xi \in \mathcal{X}^{s+1}} \frac{\mathcal{N}_{s+1}(\xi)^{2}}{2 \mid\|\xi\|_{s}^{2}} .
$$

We are now in position to deal with the nonlinear system:

$$
y^{\prime}=A y+V \bar{u}+F(y, \bar{u}) \in\left[\mathcal{D}\left(A^{*}\right)\right]^{\prime}, \quad y(0)=y_{0} .
$$

We are going to prove that $\||\cdot|\|_{s}^{2}$ is also a Lyapunov function for this system if we take the feedback control $\bar{u}=-\left(V^{*} \Pi\right) P_{N} y$. In that case, the above system can be rewritten as follows

$$
y^{\prime}+A_{\Pi} y=N(y), \quad y(0)=y_{0} .
$$

The well-posedness and the local stability of (71) is proved in Theorem 15 below under the following boundedness and Lipschitz properties for the nonlinear mapping $N(\cdot): \mathcal{X}^{1+s} \rightarrow \mathcal{X}^{s-1}$ :

$$
\begin{aligned}
\|N(\xi)\|_{\mathcal{X}^{s-1}} & \leq C\|\xi\|_{\mathcal{X}^{s}}\|\xi\|_{\mathcal{X}^{s+1}} \\
\|N(\xi)-N(\zeta)\|_{\mathcal{X}^{s-1}} & \leq C\left(\|\xi-\zeta\|_{\mathcal{X}^{s}}\left(\|\xi\|_{\mathcal{X}^{s+1}}+\|\zeta\|_{\mathcal{X}^{s+1}}\right)+\|\xi-\zeta\|_{\mathcal{X}^{s+1}}\left(\|\xi\|_{\mathcal{X}^{s}}+\|\zeta\|_{\mathcal{X}^{s}}\right)\right) .
\end{aligned}
$$

Notice that we do not make hypothesis on the nonlinearity $F$ but directly on the nonlinearity $N$ which is deduced from $F$ by taking $N(\xi) \stackrel{\text { def }}{=} F\left(\xi,-\left(V^{*} \Pi\right) P_{N} \xi\right)$. The assumptions on $F$ are given in the next subsection with appropriate notation (see (92) and (95)).

Theorem 15. Assume (72),(73) for some $s \in[0,1]$ and $y_{0} \in \mathcal{X}^{s}$. There exist $\rho>0$ and $\mu>0$ such that, if $\left\|\mid y_{0}\right\| \|_{s}<\mu$, then system (71) admits a solution $y_{y_{0}} \in W\left(\mathcal{X}^{s+1}, \mathcal{X}^{s-1}\right)$ such that $\left\|y_{y_{0}}\right\|_{W\left(\mathcal{X}^{s+1}, \mathcal{X}^{s-1}\right)} \leq \rho\|\| y_{0}\|\|_{s}$, which is unique within the class of functions in $L_{l o c}^{\infty}\left(\mathcal{X}^{s}\right) \cap L_{l o c}^{2}\left(\mathcal{X}^{s+1}\right)$. Moreover, every solution with an initial datum obeying

$$
\left\|y_{0} \mid\right\|_{s}<K_{s} \quad \text { where } \quad \frac{1}{2 K_{s}} \stackrel{\text { def }}{=} \sup _{0 \neq \xi \in \mathcal{X}^{1+s}} \frac{\left\langle L_{s} \xi \mid N(\xi)\right\rangle_{\mathcal{X}_{*}^{1-s}, \mathcal{X}^{s-1}}}{\|||\|_{s} \mathcal{N}_{s+1}(\xi)^{2}}
$$

is such that $t \longmapsto\left|\left\|y_{y_{0}}(t) \mid\right\|_{s}\right.$ is decreasing and we have:

$$
\begin{aligned}
\left\|y_{y_{0}}(t)\right\| \|_{s} & \leq\left\|\left|y_{0} \|\right|_{s} e^{-\left(\sigma+\beta_{s}\left(1-\left|\left\|y_{0} \mid\right\|_{s} / K_{s}\right)\right) t\right.} \quad \text { where } \quad \beta_{s} \stackrel{\text { def }}{=} \inf _{0 \neq \xi \in \mathcal{X}^{s+1}} \frac{\mathcal{N}_{s+1}(\xi)^{2}}{2 \|\left.|| \xi\right|_{s} ^{2}},\right. \\
\int_{0}^{\infty} e^{2 \sigma t} \mathcal{N}_{s+1}\left(y_{y_{0}}(t)\right)^{2} d t & \leq \frac{\left.\left\|y_{0}\right\|\right|_{s} ^{2}}{\left(1-\left\|y_{0}\right\| \|_{s} / K_{s}\right)} .
\end{aligned}
$$

Proof. First, suppose that $\left\|\left|y_{0}\right|\right\|_{s}<K_{s}$ and that $y \in L_{l o c}^{\infty}\left(\mathcal{X}^{s}\right) \cap L_{l o c}^{2}\left(\mathcal{X}^{s+1}\right)$ is a solution of (71) and let us prove that $y \in W\left(\mathcal{X}^{s+1}, \mathcal{X}^{s-1}\right)$ as well as estimates (74) and (75). First, since (72) ensures that $N(y) \in L_{l o c}^{2}\left(\mathcal{X}^{s-1}\right)$, from (71) we obtain $y \in W_{l o c}\left(\mathcal{X}^{s+1}, \mathcal{X}^{s-1}\right)$, and by multiplying the first equality in (71) by $L_{s} y(t)$ and using (68) and (72), we obtain:

$$
\frac{d}{d t}\||y(t)|\|_{s}^{2}+2 \sigma\left|\|y(t) \mid\|_{s}^{2}+\left(1-\left|\|y(t) \mid\|_{s} / K_{s}\right) \mathcal{N}_{s+1}(y(t))^{2} \leq 0 .\right.\right.
$$


Thus, because $\left\|\left|y_{0}\right|\right\|_{s}<K_{s}$, the mapping $t \longmapsto\left|\|y(t) \mid\|_{s}\right.$ is a nonincreasing function lower than $K_{s}$ and:

$$
\frac{d}{d t}\|\| y(t)||_{s}^{2}+2 \sigma\|\| y(t) \mid \|_{s}^{2}+\left(1-\left\|\left|y_{0}\right|\right\|_{s} / K_{s}\right) \mathcal{N}_{s+1}(y(t))^{2} \leq 0 .
$$

Then (74) follows from $2 \beta_{s}\|\mid y(t)\|_{s}^{2} \leq \mathcal{N}_{s+1}(y(t))^{2}$ and by multiplying the inequality by $e^{2 \sigma t}$ and integrating over $(0, \infty)$ gives $(75)$. Moreover, let us also notice that combining the first equation in (71) with (72) yields $\left\|y^{\prime}(t)\right\|_{\mathcal{X}^{s-1}}^{2} \leq C\|y(t)\|_{\mathcal{X}^{s+1}}^{2}$, and with (75) it provides $C_{s}>0$ such that:

$$
\|y\|_{W\left(\mathcal{X}^{s+1}, \mathcal{X}^{s-1}\right)}^{2} \leq \frac{C_{s}}{1-\left\|y_{0}\right\| \|_{s} / K_{s}}\left\|y_{0}\right\|_{s}^{2} .
$$

Next, let us set $W_{s} \stackrel{\text { def }}{=} W\left(\mathcal{X}^{s+1}, \mathcal{X}^{s-1}\right)$ for readability convenience. To prove existence and uniqueness of a solution to (71), we consider the mapping

$$
\Psi: z \in W_{s} \mapsto y_{z} \in W_{s} \quad \text { where } \quad y_{z}^{\prime}+A_{\Pi} y_{z}+N(z)=0, \quad y_{z}(0)=y_{0} .
$$

First, by combining the continuity of the mapping $y \in W_{s} \mapsto\left(y^{\prime}-A_{\Pi} y, y(0)\right) \in L^{2}\left(\mathcal{X}^{s-1}\right) \times \mathcal{X}^{s}$, which follows from (8), with (72),(73) we obtain

$$
\|\Psi(z)\|_{W_{s}} \leq C_{0}\left(\|z\|_{W_{s}}^{2}+\left\|\mid y_{0}\right\|_{s}\right) \quad \text { and } \quad\left\|\Psi\left(z_{1}\right)-\Psi\left(z_{2}\right)\right\|_{W_{s}} \leq C_{1}\left(\left\|z_{1}\right\|_{W_{s}}+\left\|z_{2}\right\|_{W_{s}}\right)\left\|z_{1}-z_{2}\right\|_{W_{s}} .
$$

Then if we assume that

$$
\rho \geq 2 C_{0}, \quad \text { and } \quad\left\|y_{0}\right\|_{s}<\min \left(\frac{1}{2 C_{0} \rho}, \frac{1}{2 C_{1} \rho}\right),
$$

then we deduce from (77) that the ball $B_{\rho} \stackrel{\text { def }}{=}\left\{z \in W_{s}\left|\|z\|_{W_{s}} \leq \rho\|\| y_{0}\right| \|_{s}\right\}$ is invariant by $\Psi$ and that the restriction of $\Psi$ to $B_{\rho}$ is a contraction. As a consequence, (71) admits a unique solution in $B_{\rho}$. If we impose moreover that

$$
\rho \geq \sqrt{2 C_{s}} \text { and }\left\|\mid y_{0}\right\|_{s} \leq \frac{K_{s}}{2},
$$

we notice that (76) ensures that every solution in $L_{l o c}^{\infty}\left(\mathcal{X}^{s}\right) \cap L_{l o c}^{2}\left(\mathcal{X}^{s+1}\right)$ belongs to $B_{\rho}$, and then must coincide with the fixed-point solution. It means that for $\rho=\max \left(\sqrt{2 C_{s}}, 2 C_{0}\right)$ and $\left\|\left|y_{0}\right|\right\|_{s}<\mu=\min \left(\frac{1}{2 C_{0} \rho}, \frac{1}{2 C_{1} \rho}\right)$ there exists a fixed point solution of (71) which is unique within the class of functions in $L_{l o c}^{\infty}\left(\mathcal{X}^{s}\right) \cap L_{l o c}^{2}\left(\mathcal{X}^{s+1}\right)$.

\subsection{Feedback stabilization with finite-dimensional dynamical controllers}

We are now interested in stabilizing the solution to $(56)$ by means of a control $\bar{u}=\left(u_{1}, \ldots, u_{K}\right) \in L^{2}\left(\mathbb{R}^{K}\right)$ which is solution to the dynamical system:

$$
\bar{u}^{\prime}=D_{K} \bar{u}+G_{K} \bar{g}, \quad \bar{u}(0)=\bar{u}_{0},
$$

where $\bar{g} \in\left(L^{2}(\mathbb{R})\right)^{K}, D_{K} \in \mathcal{L}\left(\mathbb{R}^{K}\right)$ and $G_{K} \in \mathcal{L}\left(\mathbb{R}^{K}\right)$ is invertible. We introduce the new state $Y={ }^{t}(y, \bar{u})$, the new initial datum $Y_{0}={ }^{t}\left(y_{0}, \bar{u}_{0}\right) \in \mathcal{X} \times \mathbb{R}^{K}$ and the following extended linear operator on $\mathcal{X} \times \mathbb{R}^{K}$ :

$$
\mathbb{A} \stackrel{\text { def }}{=}\left(\begin{array}{cc}
A & V \\
0 & D_{K}
\end{array}\right) \quad \text { and } \quad \mathcal{D}(\mathbb{A}) \stackrel{\text { def }}{=}\left\{Y \in \mathcal{X} \times \mathbb{R}^{K} \mid \mathbb{A} Y \in \mathcal{X} \times \mathbb{R}^{K}\right\},
$$

so that system (56), (79) can be rewritten as follows:

$$
Y^{\prime}=\mathbb{A} Y+\mathbb{B} \bar{g}, \quad Y(0)=Y_{0} \quad \text { where } \quad \mathbb{B} \stackrel{\text { def }}{=}\left(\begin{array}{c}
0 \\
G_{K}
\end{array}\right) .
$$

Proposition 16. The unbounded operator $(\mathcal{D}(\mathbb{A}), \mathbb{A})$ is the infinitesimal generator of an analytic semigroup on $\mathcal{X} \times \mathbb{R}^{K}$. The adjoint of $\mathbb{A}$ is given by

$$
\mathbb{A}^{*}=\left(\begin{array}{cc}
A^{*} & 0 \\
V^{*} & D_{K}^{*}
\end{array}\right) \quad \text { and } \quad \mathcal{D}\left(\mathbb{A}^{*}\right)=\mathcal{D}\left(A^{*}\right) \times \mathbb{R}^{K}
$$

and there exists $\lambda_{0}>0$ such that $\widehat{\mathbb{A}} \stackrel{\text { def }}{=} \lambda_{0}-\mathbb{A}$ obeys:

$$
\begin{aligned}
\mathcal{D}\left(\widehat{\mathbb{A}}^{\theta}\right) & =[\mathcal{D}(\mathbb{A}), \mathcal{X}]_{1-\theta}=\left\{Y={ }^{t}(y, \bar{u}) \in \mathcal{X} \times \mathbb{R}^{K} \mid y-\widehat{A}^{-1} V \bar{u} \in \mathcal{D}\left(\widehat{A}^{\theta}\right)\right\}, \quad \forall \theta \in[0,1], \\
\mathcal{D}\left(\widehat{\mathbb{A}}^{* \theta}\right) & =\left[\mathcal{D}\left(\mathbb{A}^{*}\right), \mathcal{X}\right]_{1-\theta}=\mathcal{D}\left(\widehat{A}^{* \theta}\right) \times \mathbb{R}^{K}, \quad \forall \theta \in[0,1] .
\end{aligned}
$$


Proof. First, by remarking that for $\lambda \in \mathbb{C}$ the equality $(\lambda-\mathbb{A})^{t}(y, \bar{u})={ }^{t}(f, \bar{h})$ is equivalent to

$$
(\lambda-A) y=V \bar{u}+f \quad \text { and } \quad\left(\lambda-D_{K}\right) \bar{u}=\bar{h},
$$

we deduce that the resolvent set of $\mathbb{A}$ is exactly the union of the resolvent sets of $A$ and of $D_{K}$. Thus, to prove that $\mathbb{A}$ generates an analytic semigroup we have to prove that there exists $M>0$ such that for all $\widetilde{F}={ }^{t}(f, \bar{h}) \in \mathcal{X} \times \mathbb{R}^{K}$ and for all $\lambda$ in an open sector of the complex plane, symmetric with respect to the axis $\mathbb{R}$ and with an opening angle greater than $\pi$ [9, Chap. II-1, Thm. 2.10], we have:

$$
\left\|(\lambda-\mathbb{A})^{-1} \widetilde{F}\right\|_{\mathcal{X} \times \mathbb{R}^{K}} \leq \frac{M}{|\lambda|}\|\widetilde{F}\|_{\mathcal{X} \times \mathbb{R}^{K}}
$$

The above estimate can be obtained by remarking that for $\lambda$ in the resolvent set of $\mathbb{A},(84)$ is equivalent to

$$
y=(\lambda-A)^{-1} \widehat{A}\left(\widehat{A}^{-1} V\right) \bar{u}+(\lambda-A)^{-1} f \quad \text { and } \quad \bar{u}=\left(\lambda-D_{K}\right)^{-1} \bar{h},
$$

and by using the boundedness of $\widehat{A}^{-1} V$ as well as resolvent estimates related to the analyticity of $\left(e^{A t}\right)_{t \geq 0}$ and of $\left(e^{D_{K} t}\right)_{t \geq 0}$. Thus, we can choose $\lambda_{0}>0$ large enough so that the positive halfaxis $\mathbb{R}^{+}$is contained in the resolvent set of $\widehat{A} \stackrel{\text { def }}{=} \lambda_{0}-A$ and of $\widehat{D}_{K} \stackrel{\text { def }}{=} \lambda_{0}-D_{K}$.

Next, in order to prove the first equalities in $(82),(83)$, let us recall that if $L$ is a closed linear mapping on a Hilbert space $\mathcal{H}$ such that $-L$ is the infinitesimal generator of an analytic semigroup of negative type then the interpolation equalities $[\mathcal{D}(L), \mathcal{X}]_{1-\theta}=\mathcal{D}\left(L^{\theta}\right)$ and $\left[\mathcal{D}\left(L^{*}\right), \mathcal{X}\right]_{1-\theta}=\mathcal{D}\left(L^{* \theta}\right)$ are equivalent to the fact that the operators defined for $z \in \mathbb{C}$ such that $\Re z>0$ as follows

$$
L^{-z}=\frac{\sin \pi z}{\pi} \int_{0}^{+\infty} t^{-z}(t+L)^{-1} d t
$$

can be extended to strongly continuous functions from $\{z \in \mathbb{C} \mid \Re z \geq 0\}$ to $\mathcal{L}(\mathcal{H})$, see [9, Chap. II-1, Thm. 6.1] or [40]. Then, since we have the complex interpolation equalities $[\mathcal{D}(\widehat{A}), \mathcal{X}]_{1-\theta}=\mathcal{D}\left(\widehat{A}^{\theta}\right)$ and $\left[\mathcal{D}\left(\widehat{D}_{K}\right), \mathcal{X}\right]_{1-\theta}=$ $\mathcal{D}\left(\widehat{D}_{K}^{\theta}\right)$ for $\theta \in(0,1)$, and since easy calculations give

$$
(t+\widehat{\mathbb{A}})^{-1}=\left(\begin{array}{cc}
(t+\widehat{A})^{-1} & -(t+\widehat{A})^{-1} V\left(t+\widehat{D}_{K}\right)^{-1} \\
0 & \left(t+\widehat{D}_{K}\right)^{-1}
\end{array}\right)
$$

and

$$
\widehat{\mathbb{A}}^{-z}=\left(\begin{array}{cc}
\widehat{A}^{-z} & -\beta(z) \\
0 & \widehat{D}_{K}^{-z}
\end{array}\right) \text { where } \quad \beta(z)=\frac{\sin \pi z}{\pi} \int_{0}^{+\infty} t^{-z}(t+\widehat{A})^{-1} V\left(t+\widehat{D}_{K}\right)^{-1} d t
$$

then to prove the first equalities in $(82),(83)$ it remains to prove that we can extend $\beta(z)$ to a strongly continuous function from $\{z \in \mathbb{C} \mid \Re(z) \geq 0\}$ in $\mathcal{L}\left(\mathbb{R}^{K}, \mathcal{X}\right)$. From $\widehat{A}(t+\widehat{A})^{-1}=I-t(t+\widehat{A})^{-1}$ and $\left\|(t+\widehat{A})^{-1}\right\|_{\mathcal{L}(\mathcal{X})} \leq$ $C_{0}(1+t)^{-1}$ we deduce that $\left\|(t+\widehat{A})^{-1}\right\|_{\mathcal{L}(\mathcal{X}, \mathcal{D}(\widehat{A}))}=\left\|\widehat{A}(t+\widehat{A})^{-1}\right\|_{\mathcal{L}(\mathcal{X})} \leq 1+C_{0}$ and an interpolation argument with $[\mathcal{D}(\widehat{A}), \mathcal{X}]_{1-\gamma}=\mathcal{D}\left(\widehat{A}^{\gamma}\right)$ gives $\left\|(t+\widehat{A})^{-1}\right\|_{\mathcal{L}\left(\mathcal{X}, \mathcal{D}\left(\widehat{A}^{\gamma}\right)\right)}=\left\|\widehat{A}^{\gamma}(t+\widehat{A})^{-1}\right\|_{\mathcal{L}(\mathcal{X})} \leq\left(1+C_{0}\right)^{\gamma}\left(C_{0}(1+t)^{-1}\right)^{1-\gamma}$. Then $(1+t)^{1-\gamma} \widehat{A}^{\gamma}(t+\widehat{A})^{-1}$ is bounded independently of $t$ and with $\widehat{A}^{-\gamma} V \in \mathcal{L}(\mathcal{X})$ we can bound the term under the integral and obtain that $\beta(z)$ is bounded independently on $z \in\{z \in \mathbb{C} \mid \Re(z)>0\}$ in a neighborhood of 0 . Then by [27, Ch. 17 , Thm. 17.9.1] one can extend $\beta(z)$ to a strongly continuous function from $\{z \in \mathbb{C} \mid \Re(z) \geq 0\}$ in $\mathcal{L}\left(\mathbb{R}^{K}, \mathcal{X}\right)$

Next, if we remark that $\mathcal{D}(\mathbb{A})$ is composed with ${ }^{t}(y, \bar{u}) \in \mathcal{X} \times \mathbb{R}^{K}$ such that $A y+V \bar{u} \in \mathcal{X}$, then we have

$$
\mathcal{D}(\mathbb{A})=\left\{{ }^{t}(y, \bar{u}) \in \mathcal{X} \times \mathbb{R}^{K} \mid y-\widehat{A}^{-1} V \bar{u} \in \mathcal{D}(A)\right\},
$$

and with $[\mathcal{D}(A), \mathcal{X}]_{1-\theta}=\mathcal{D}\left(\widehat{A}^{\theta}\right)$ for $\theta \in(0,1)$ we obtain the second equality in (82) from an interpolation argument.

Finally, to characterize the adjoint of $\mathbb{A}$ let us show the inclusion $\mathcal{D}\left(\mathbb{A}^{*}\right) \subset \mathcal{D}\left(A^{*}\right) \times \mathbb{R}^{K}$ which is the only non obvious fact to prove. If $Y={ }^{t}(y, \bar{u}) \in \mathcal{D}\left(\mathbb{A}^{*}\right)$ then $Z \in \mathcal{D}(\mathbb{A}) \mapsto(\mathbb{A} Y \mid Z)_{\mathcal{X} \times \mathbb{R}^{K}}$ is continuous for the topology of $\mathcal{X} \times \mathbb{R}^{K}$, and by remarking that $\mathcal{D}(A) \times\{0\} \subset \mathcal{D}(\mathbb{A})$ we deduce that $z \in \mathcal{D}(A) \mapsto(A y \mid z)_{\mathcal{X}}$ is continuous for the topology of $\mathcal{X}$. Then it means that $y \in \mathcal{D}\left(A^{*}\right)$ and the desired inclusion is proved. Notice that the second equality in (83) follows from (54) by interpolation. 
We now consider system (56) with a control function solution to the dynamical system:

$$
\bar{u}^{\prime}=D_{K} \bar{u}-G_{K} G_{K}^{*} \Pi_{2} y_{N}-G_{K} G_{K}^{*} \Pi_{3} \bar{u}, \quad \bar{u}(0)=\bar{u}_{0},
$$

where $\Pi_{2}$ and $\Pi_{3}$ are components of the Riccati operator $\Pi$ introduced in Subsection 3.5. Such a system can be rewritten as:

$$
Y^{\prime}=\mathbb{A} Y-\mathbb{B B}^{*} \Pi \mathbb{P}_{N} Y, \quad Y(0)=Y_{0} \quad \text { where } \quad \mathbb{P}_{N}=\left(\begin{array}{cc}
P_{N} & 0 \\
0 & \mathrm{I}_{\mathbb{R}^{K}}
\end{array}\right) .
$$

Proposition 17. Assume that $G_{K} \in \mathcal{L}\left(\mathbb{R}^{K}\right)$ is invertible. For $Y_{0} \in \mathcal{X} \times \mathbb{R}^{K}$ the solution of (86) is given by $Y(t)=e^{-\mathbb{A}_{\Pi} t} Y_{0}$, where $\left(e^{-\mathbb{A}_{\Pi} t}\right)_{t \geq 0}$ is an analytic semigroup on $\mathcal{X} \times \mathbb{R}^{K}$ with infinitesimal generator $-\mathbb{A}_{\boldsymbol{\Pi}}$ defined by

$$
\mathcal{D}\left(\mathbb{A}_{\Pi}\right)=\mathcal{D}(\mathbb{A}) \quad \text { and } \quad \mathbb{A}_{\Pi} \xi=\mathbb{B B}^{*} \Pi \mathbb{P}_{N} \xi-\mathbb{A} \xi
$$

The semigroup $\left(e^{-\mathbb{A}_{\Pi} t}\right)_{t \geq 0}$ is exponentially stable with an exponential rate strictly lower than $-\sigma$ :

$$
\inf \left\{\Re \lambda \mid \lambda \in \sigma\left(\mathbb{A}_{\Pi}\right)\right\}>\sigma .
$$

Moreover, the adjoint of $\mathbb{A}_{\Pi}$ is given by

$$
\mathcal{D}\left(\mathbb{A}_{\Pi}^{*}\right)=\mathcal{D}\left(\mathbb{A}^{*}\right) \quad \text { and } \quad \mathbb{A}_{\Pi}^{*} \xi=\mathbb{P}_{N}^{*}\left(\mathbb{B}^{*} \Pi\right)^{*} \mathbb{B}^{*} \xi-\mathbb{A}^{*} \xi,
$$

and for all $\theta \in[0,1]$ the following equalities hold:

$$
\mathcal{D}\left(\mathbb{A}_{\Pi}^{\theta}\right)=\mathcal{D}\left(\widehat{\mathbb{A}}^{\theta}\right) \quad \text { and } \quad \mathcal{D}\left(\mathbb{A}_{\Pi}^{* \theta}\right)=\mathcal{D}\left(\widehat{\mathbb{A}}^{* \theta}\right) .
$$

Proof. First, the boundedness of $\mathbb{B B}^{*} \Pi \mathbb{P}_{N}$ ensures that $\mathbb{A}_{\Pi}-\mathbb{B B}^{*} \Pi \mathbb{P}_{N}$ and $\mathbb{A}$ have the same domain and the analyticity of $\left(e^{-\mathbb{A}_{\Pi} t}\right)_{t \geq 0}$ is a consequence of the analyticity of $\left(e^{\mathbb{A} t}\right)_{t \geq 0}$, see [31, Chap. 3, Cor. 2.2, p. 81]. Thus, similarly as in Subsection 4.1 we can prove that there is $a>0$ such that

$$
\left\|y_{N}^{-}(t)\right\|_{\mathcal{X}} \leq C\left(\left\|y_{0}\right\|_{\mathcal{X}}+\left\|\bar{u}_{0}\right\|_{\mathbb{R}^{K}}\right) e^{-(\sigma+a) t},
$$

which, with (52), gives the exponential stability of $\left(e^{-\mathbb{A}_{\Pi} t}\right)_{t \geq 0}$. Finally, from (82) and (83) with a perturbation argument $\left[17\right.$, Prop. 2.7] we deduce the interpolation equalities $\mathcal{D}\left(\mathbb{A}_{\Pi}^{\theta}\right)=\left[\mathcal{D}\left(\mathbb{A}_{\Pi}\right), \mathcal{X}\right]_{1-\theta}$ and $\mathcal{D}\left(\mathbb{A}_{\Pi}^{* \theta}\right)=\left[\mathcal{D}\left(\mathbb{A}_{\Pi}^{*}\right), \mathcal{X}\right]_{1-\theta}$, and (90) follows from $(82),(83)$ and $(87),(89)$.

Next, we recall that $G \in \mathcal{L}(\mathcal{X}, \mathcal{O})$ is an operator satisfying (55) and we define an analogue extended operator $\mathbb{G} \in \mathcal{L}\left(\mathcal{X} \times \mathbb{R}^{K}, \mathcal{O} \times \mathbb{R}^{K}\right)$ which is boundedly invertible and which obeys $\mathbb{G}^{*} \mathbb{G} \in \mathcal{L}\left(\mathcal{D}\left(\mathbb{A}_{\Pi}^{\frac{1}{2}}\right), \mathcal{D}\left(\mathbb{A}_{\Pi}^{* \frac{1}{2}}\right)\right)$ as follows:

$$
\mathbb{G}\left(\begin{array}{c}
y \\
\bar{u}
\end{array}\right)=\left(\begin{array}{c}
G\left(y-\widehat{A}^{-1} V \bar{u}\right) \\
\bar{u}
\end{array}\right) .
$$

The bounded invertibility of $\mathbb{G}$ is an easy consequence of the bounded invertibility of $G$. To prove the second fact, we first notice $\mathcal{D}\left(\widehat{\mathbb{A}}^{\frac{1}{2}}\right)=\mathcal{D}\left(\mathbb{A}_{\Pi}^{\frac{1}{2}}\right)$ and $\mathcal{D}\left(\widehat{\mathbb{A}}^{* \frac{1}{2}}\right)=\mathcal{D}\left(\mathbb{A}_{\Pi}^{* \frac{1}{2}}\right)$. Then, the fact that $\mathbb{G}^{*} \mathbb{G} \in \mathcal{L}\left(\mathcal{D}\left(\widehat{\mathbb{A}}^{\frac{1}{2}}\right), \mathcal{D}\left(\widehat{\mathbb{A}}^{* \frac{1}{2}}\right)\right)$ is a consequence of $G^{*} G \in \mathcal{L}\left(\mathcal{D}\left(\widehat{A}^{\frac{1}{2}}\right), \mathcal{D}\left(\widehat{A}^{* \frac{1}{2}}\right)\right)$ and of the expression:

$$
\mathbb{G}^{*} \mathbb{G}\left(\begin{array}{c}
y \\
\bar{u}
\end{array}\right)=\left(\begin{array}{c}
G^{*} G\left(y-\widehat{A}^{-1} V \bar{u}\right) \\
\bar{u}-V^{*} \widehat{A}^{*-1} G^{*} G\left(y-\widehat{A}^{-1} V \bar{u}\right)
\end{array}\right) .
$$

Then we can proceed as in Subsection 4.1: we define the following equivalent norms on $\mathcal{D}\left(\widehat{\mathbb{A}}^{\frac{s}{2}}\right)$ and on $\mathcal{D}\left(\widehat{\mathbb{A}}^{\frac{s}{2}+\frac{1}{2}}\right)$ for $s \in[0,1]$

$$
\||\cdot|\|_{s}^{2} \stackrel{\text { def }}{=} \int_{0}^{\infty} e^{2 \sigma t}\left\|\mathbb{G}_{\mathbb{\Pi}^{\frac{1}{2}+\frac{s}{2}}} e^{-\mathbb{A}_{\Pi} t} \cdot\right\|_{\mathcal{O} \times \mathbb{R}^{K}}^{2} d t=\left\langle\mathbb{L}_{s} \cdot \mid \cdot\right\rangle_{\left[\mathcal{D}\left(\widehat{\mathbb{A}}^{\frac{s}{2}}\right)\right]^{\prime}, \mathcal{D}\left(\widehat{\mathbb{A}}^{\frac{s}{2}}\right)},
$$

and

$$
\mathcal{N}_{s+1}(\cdot)^{2} \stackrel{\text { def }}{=}\left\|\mathbb{G A}_{\Pi}^{\frac{1}{2}+\frac{s}{2}} \cdot\right\|_{\mathcal{O} \times \mathbb{R}^{K}}^{2}=2\left\langle\mathbb{L}_{s} \cdot \mid \mathbb{A}_{\Pi} \cdot\right\rangle_{\mathcal{D}\left(\widehat{\mathbb{A}}^{* \frac{1}{2}-\frac{s}{2}}\right), \mathcal{D}\left(\widehat{\mathbb{A}}^{\frac{s}{2}+\frac{s}{2}}\right)}-2 \sigma|\|\cdot\||_{s}^{2} .
$$


Thus, we prove a stabilization theorem for a nonlinear system of type:

$$
Y^{\prime}+\mathbb{A}_{\Pi} Y=N(Y), \quad Y(0)=Y_{0}
$$

provided that the nonlinear mapping $N(\cdot): \mathcal{D}\left(\widehat{\mathbb{A}}^{\frac{s}{2}+\frac{1}{2}}\right) \rightarrow\left[\mathcal{D}\left(\widehat{\mathbb{A}}^{*\left(\frac{1}{2}-\frac{s}{2}\right)}\right)\right]^{\prime}$ satisfies

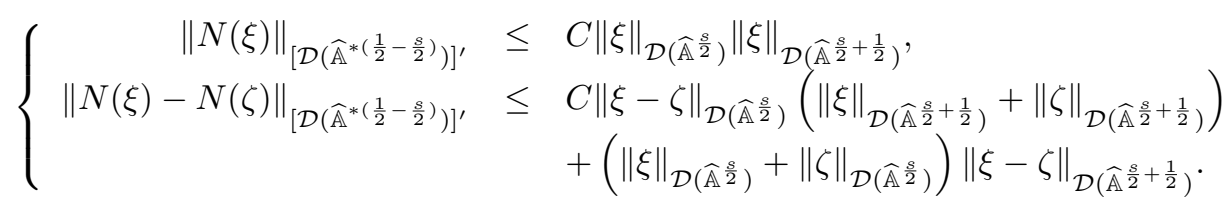

Theorem 18. Assume that $G_{K} \in \mathcal{L}\left(\mathbb{R}^{K}\right)$ is invertible and assume (92) for some $s \in[0,1]$ and $y_{0} \in \mathcal{D}\left(\widehat{\mathbb{A}}^{\frac{s}{2}}\right)$. There exist $\rho>0$ and $\mu>0$ such that, if $\left\|\mid Y_{0}\right\|_{s}<\mu$, then system (91) admits a solution

$$
Y_{Y_{0}} \in W\left(\mathcal{D}\left(\widehat{\mathbb{A}}^{\frac{s}{2}+\frac{1}{2}}\right),\left[\mathcal{D}\left(\widehat{\mathbb{A}}^{*\left(\frac{1}{2}-\frac{s}{2}\right)}\right)\right]^{\prime}\right)
$$

such that

$$
\left\|Y_{Y_{0}}\right\|_{W\left(\mathcal{D}\left(\widehat{\mathbb{A}}^{\frac{s}{2}+\frac{1}{2}}\right),\left[\mathcal{D}\left(\widehat{\mathbb{A}}^{*\left(\frac{1}{2}-\frac{s}{2}\right)}\right)\right]^{\prime}\right)} \leq \rho\|\| Y_{0} \|_{s},
$$

which is unique within the class of functions in $L_{l o c}^{\infty}\left(\mathcal{D}\left(\widehat{\mathbb{A}}^{\frac{s}{2}}\right)\right) \cap L_{\text {loc }}^{2}\left(\mathcal{D}\left(\widehat{\mathbb{A}}^{\frac{s}{2}}+\frac{1}{2}\right)\right)$. Moreover, every solution with an initial datum obeying

$$
\left\|Y_{0}\right\|_{s}<K_{s} \quad \text { where } \quad \frac{1}{2 K_{s}} \stackrel{\text { def }}{=} \sup _{0 \neq \xi \in \mathcal{D}\left(\widehat{\mathbb{A}}^{\frac{s}{2}}+\frac{1}{2}\right)} \frac{\left\langle\mathbb{L}_{s} \xi \mid N(\xi)\right\rangle_{\left[\mathcal{D}\left(\widehat{\mathbb{A}}^{\frac{s}{2}}\right)\right]^{\prime}, \mathcal{D}\left(\widehat{\mathbb{A}}^{\frac{s}{2}}\right)}}{\||| \xi \mid\|_{s} \mathcal{N}_{s+1}(\xi)^{2}},
$$

is such that $t \longmapsto||\left|Y_{Y_{0}}(t)\right| \|_{s}$ is decreasing and we have:

$$
\begin{aligned}
& \left.\left\|Y_{Y_{0}}(t)\left|\left\|_{s} \leq\right\| Y_{0}\right|\right\|\right|_{s} e^{-\left(\sigma+\beta_{s}\left(1-\left\||| Y_{0} \mid\right\|_{s} / K_{s}\right)\right) t} \quad \text { where } \quad \beta_{s} \stackrel{\text { def }}{=} \inf _{0 \neq \xi \in \mathcal{D}\left(\widehat{\mathbb{A}}^{\frac{s}{2}+\frac{1}{2}}\right)} \frac{\mathcal{N}_{s+1}(\xi)^{2}}{2|| \xi \xi||_{s}^{2}} \\
& \int_{0}^{\infty} e^{2 \sigma t} \mathcal{N}_{s+1}\left(Y_{Y_{0}}(t)\right)^{2} d t \leq \frac{\left\|\left|Y_{0}\right|\right\|_{s}^{2}}{\left(1-\left\||| Y_{0}\right\|_{s} / K_{s}\right)}
\end{aligned}
$$

Remark 19. In practice, we do not put any nonlinearity in the equation of $\bar{u}$ and thus the nonlinearity mapping has the form

$$
N\left(\begin{array}{c}
y \\
\bar{u}
\end{array}\right)=\left(\begin{array}{c}
F(y, \bar{u}) \\
0
\end{array}\right)
$$

with $N$ satisfying (92).

\section{Stabilization of the Navier-Stokes system}

\subsection{Notations and Functional Framework}

We suppose here that $\Omega$ is an open subset of $\mathbb{R}^{d}, d=2,3$, with a boundary $\partial \Omega$ of class $C^{2,1}$, we denote by $L^{2}(\Omega)$, $L^{2}(\partial \Omega), H^{s}(\Omega), H^{s}(\partial \Omega), H_{0}^{s}(\Omega)$ and $H^{-s}(\Omega)=\left(H_{0}^{s}(\Omega)\right)^{\prime}$ for $s \geq 0$, the usual Lebesgue and Sobolev spaces of scalar functions in $\Omega$ or in $\partial \Omega$, and we write in bold the spaces of vector-valued functions: $\mathbf{L}^{2}(\Omega)=\left(L^{2}(\Omega)\right)^{d}$, $\mathbf{L}^{2}(\partial \Omega)=\left(L^{2}(\partial \Omega)\right)^{d}$, etc. We also introduce different spaces of free divergence functions and some corresponding trace spaces:

$$
\begin{aligned}
\mathbf{V}^{s}(\Omega) & \stackrel{\text { def }}{=}\left\{y \in \mathbf{H}^{s}(\Omega) ; \nabla \cdot y=0 \text { in } \Omega, \quad\langle y \cdot n, 1\rangle_{H^{-\frac{1}{2}}(\partial \Omega), H^{\frac{1}{2}}(\partial \Omega)}=0\right\} \quad \text { for } s \geq 0, \\
\mathbf{V}_{n}^{s}(\Omega) & \stackrel{\text { def }}{=}\left\{y \in \mathbf{H}^{s}(\Omega) ; \nabla \cdot y=0 \text { in } \Omega, \quad y \cdot n=0 \quad \text { on } \partial \Omega\right\} \quad \text { for } s \geq 0, \\
\mathbf{V}_{0}^{s}(\Omega) & \stackrel{\text { def }}{=}\left\{y \in \mathbf{H}^{s}(\Omega) ; \nabla \cdot y=0 \text { in } \Omega, \quad y=0 \quad \text { on } \partial \Omega\right\} \quad \text { for } s>\frac{1}{2}, \\
\mathbf{V}^{s}(\partial \Omega) & \stackrel{\text { def }}{=}\left\{y \in \mathbf{H}^{s}(\partial \Omega) ;\langle y \cdot n, 1\rangle_{H^{-\frac{1}{2}}(\partial \Omega), H^{\frac{1}{2}}(\partial \Omega)}=0\right\} \quad \text { for } s \geq-\frac{1}{2} .
\end{aligned}
$$


Moreover, we also define $\mathbf{V}_{0}^{s}(\Omega)$ for $s \in\left[0,1 / 2\left[\right.\right.$ by $\mathbf{V}_{0}^{s}(\Omega)=\mathbf{V}_{n}^{s}(\Omega)$, for $s=1 / 2$ by $\mathbf{V}_{0}^{\frac{1}{2}}(\Omega)=\left\{y \in \mathbf{V}_{n}^{\frac{1}{2}}(\Omega) \mid y \in\right.$ $\left.\mathbf{L}_{-\frac{1}{2}}^{2}(\Omega)\right\}$ where $\mathbf{L}_{-\frac{1}{2}}^{2}(\Omega)$ is the space of functions $y \in \mathbf{L}^{2}(\Omega)$ such that $\int_{\Omega} \operatorname{dist}(x, \partial \Omega)^{-1}|y|^{2} d x<+\infty$ and for $s<0$ by $\mathbf{V}_{0}^{s}(\Omega)=\left[\mathbf{V}_{0}^{-s}(\Omega)\right]^{\prime}$. Notice that the subscript 0 in $\mathbf{V}_{0}^{s}(\Omega)$ only means that one may have a vanishing Dirichlet boundary condition.

We denote by $P$ the orthogonal projector from $\mathbf{L}^{2}(\Omega)$ onto $\mathbf{V}_{n}^{0}(\Omega)$ [21, Chap. III, Thm. 1.1]. Notice that since $\Omega$ is of class $C^{2,1}$, by studying its related Neumann problem, see [21, Chap.III, Lem 1.2] and [23, Chap. I, Thm. 1.10], we also have for all $s \in[0,2]$ :

$$
P \in \mathcal{L}\left(\mathbf{H}^{s}(\Omega), \mathbf{V}_{n}^{s}(\Omega)\right)
$$

Moreover, $P$ can also be continuously extended to a bounded linear operator from $\mathbf{H}^{-1}(\Omega)$ onto $\mathbf{V}_{0}^{-1}(\Omega)[6$, App. A].

In order to rewrite the controlled Oseen or Navier-Stokes system as an evolution equation by using a semigroup approach, let us introduce the Oseen operator $A$ on $\mathbf{V}_{n}^{0}(\Omega)$ :

$$
\left\{\begin{aligned}
\mathcal{D}(A) & \stackrel{\text { def }}{=}\left\{y \in \mathbf{V}_{0}^{1}(\Omega) \mid P\left(\nu \Delta y-\left(w_{S} \cdot \nabla\right) y-(y \cdot \nabla) w_{S}\right) \in \mathbf{V}_{n}^{0}(\Omega)\right\} \\
A y & \stackrel{\text { def }}{=} P\left(\nu \Delta y-\left(w_{S} \cdot \nabla\right) y-(y \cdot \nabla) w_{S}\right) .
\end{aligned}\right.
$$

Following [34], there exists $\lambda_{0}>0$ such that $\lambda_{0} \in \rho(A)$ and

$$
\left(\left(\lambda_{0} I-A\right) y, y\right)_{\mathbf{V}_{n}^{0}(\Omega)} \geq \frac{\nu}{2}\|y\|_{\mathbf{V}_{0}^{1}(\Omega)}^{2} \quad \forall y \in \mathcal{D}(A),
$$

and the following theorem holds.

Theorem 20. The Oseen operator $(97)$ obeys $\mathcal{D}(A)=\mathbf{V}_{0}^{2}(\Omega)$, its adjoint is given by

$$
\mathcal{D}\left(A^{*}\right)=\mathbf{V}_{0}^{2}(\Omega) \quad \text { and } \quad A^{*} y=P\left(\nu \Delta y+\left(w_{S} \cdot \nabla\right) y-{ }^{t}\left(\nabla w_{S}\right) y\right),
$$

and the resolvent of $(\mathcal{D}(A), A)$ (resp. $\left.\left(\mathcal{D}\left(A^{*}\right), A^{*}\right)\right)$ is compact. Moreover, $(\mathcal{D}(A), A)$ (resp. $\left.\left(\mathcal{D}\left(A^{*}\right), A^{*}\right)\right)$ is the infinitesimal generator of an analytic semigroup on $\mathbf{V}_{n}^{0}(\Omega)$ and the characterization below holds:

$$
\mathcal{D}\left(\left(\lambda_{0}-A\right)^{\theta}\right)=\mathcal{D}\left(\left(\lambda_{0}-A^{*}\right)^{\theta}\right)=\left[\mathbf{V}_{0}^{2}(\Omega), \mathbf{V}_{n}^{0}(\Omega)\right]_{1-\theta}=\mathbf{V}_{0}^{2 \theta}(\Omega) \quad \text { for all } \theta \in[0,1] .
$$

In the above setting ${ }^{t}(E)$ denotes the transpose of the matrix $E$.

Proof of Theorem 20. First, since regularity result for the Stokes system [21, Chap. IV, Thm. 6.1, p. 231] ensures that the domain of the Stokes operator (defined by $(97)$ for $w_{S}=0$ ) is equal to $\mathbf{V}_{0}^{2}(\Omega)$, then $\mathcal{D}(A)=$ $\mathbf{V}_{0}^{2}(\Omega)$ follows from a perturbation argument [31, Chap. 3, Cor. 2.4 p. 81]. The characterization of $A^{*}$ follows analogously with an integration by parts. Thus, the compactness of the resolvent of $(\mathcal{D}(A), A)$ and of $\left(\mathcal{D}\left(A^{*}\right), A^{*}\right)$ is a consequence of the compact embedding $\mathbf{V}_{0}^{2}(\Omega) \subset \subset \mathbf{V}_{n}^{0}(\Omega)$. Next, the fact that $A$ and $A^{*}$ are infinitesimal generators of analytic semigroups on $\mathbf{V}_{n}^{0}(\Omega)$ is a consequence of (98) with [9, Part. II, Chap. 1, Thm 2.12]. Finally, (99) follows by interpolation: since $\lambda_{0}-A$ is accretive and boundedly invertible with domain $\mathbf{V}_{0}^{2}(\Omega)$ we have $\mathcal{D}\left(\left(\lambda_{0}-A\right)^{\theta}\right)=\left[\mathbf{V}_{0}^{2}(\Omega), \mathbf{V}_{n}^{0}(\Omega)\right]_{1-\theta}$ and $\mathcal{D}\left(\left(\lambda_{0}-A^{*}\right)^{\theta}\right)=\left[\mathbf{V}_{0}^{2}(\Omega), \mathbf{V}_{n}^{0}(\Omega)\right]_{1-\theta}[9$, Part. II, Chap. 1, Prop. 6.1]. Finally, since we can construct a projection operator $P_{0}: \mathbf{L}^{2}(\Omega) \rightarrow \mathbf{V}_{n}^{0}(\Omega)$ which is also continuous from $\mathbf{H}^{2}(\Omega) \cap \mathbf{H}_{0}^{1}(\Omega)$ onto $\mathbf{V}_{0}^{2}(\Omega)$ (for instance by setting $P_{0}=A_{0}^{-1} P \Delta$ where $A_{0}$ is the Stokes operator, see [19]) then by $\left[38\right.$, Thm. 1.17.1.1, p.118] we deduce that $\left[\mathbf{V}_{0}^{2}(\Omega), \mathbf{V}_{n}^{0}(\Omega)\right]_{1-\theta}=\left[\mathbf{H}^{2}(\Omega) \cap \mathbf{H}_{0}^{1}(\Omega), \mathbf{L}^{2}(\Omega)\right]_{1-\theta} \cap \mathbf{V}_{n}^{0}(\Omega)$ and the characterization of $\left[\mathbf{H}^{2}(\Omega) \cap \mathbf{H}_{0}^{1}(\Omega), \mathbf{L}^{2}(\Omega)\right]_{1-\theta}$ obtained from [38, Thm. 4.3.3.1, p.321], or from [24], allows to conclude.

We then first consider Oseen $(\kappa=0)$ or Navier-Stokes $(\kappa=1)$ system with an internal control locally supported in an open subset $\omega$ of $\Omega$ :

$$
\left\{\begin{array}{rlll}
\frac{\partial w}{\partial t}-\nu \Delta w+\left(w_{S} \cdot \nabla\right) w+(w \cdot \nabla) w_{S}+\kappa(w \cdot \nabla) w+\nabla p & =\mathbb{1}_{\omega} h & \text { in } & (0, \infty) \times \Omega, \\
\nabla \cdot w & =0 & \text { in } & (0, \infty) \times \Omega, \\
w & =0 & \text { on } & (0, \infty) \times \partial \Omega, \\
w(0) & =w_{0} & \text { in } & \Omega .
\end{array}\right.
$$

In the above setting, $\mathbb{1}_{\omega}$ is the characteristic function of $\omega$ and $h \in L_{l o c}^{2}\left(0, \infty ; \mathbf{L}^{2}(\Omega)\right)$ is a control function. We shall say that a pair $(w, p)$ is a solution of $(100)$ if $w \in L_{l o c}^{2}\left((0, \infty) ; \mathbf{H}^{1}(\Omega)\right) \cap C\left([0, \infty) ; \mathbf{L}^{2}(\Omega)\right)$, if $p$ is 
a distribution in $\mathcal{D}^{\prime}\left((0, \infty) ; L^{2}(\Omega)\right)$, and if $(w, p)$ satisfies the first equation in (100) as an equality in the distribution space $\mathcal{D}^{\prime}\left(0, \infty ; \mathbf{H}^{-1}(\Omega)\right)$, and the second, third and fourth equations in (100) as equalities in $L_{\text {loc }}^{2}\left(L^{2}(\Omega)\right)$, in $L^{2}\left(\mathbf{L}^{2}(\partial \Omega)\right)$ and in $\mathbf{L}^{2}(\Omega)$ respectively. Notice that such a definition is meaningful because when $w \in L_{l o c}^{2}\left((0, \infty) ; \mathbf{H}^{1}(\Omega)\right)$ and $w_{S} \in \mathbf{V}^{1}(\Omega)$ the convective terms $\left(w_{S} \cdot \nabla\right) w,(w \cdot \nabla) w_{S}$, and in particular the nonlinear term $(w \cdot \nabla) w$, are well-defined in $L_{l o c}^{2}\left(\mathbf{H}^{-1}(\Omega)\right)$. More precisely, if $s_{1}, s_{2}$ and $s_{3}$ are real nonnegative numbers such that $s_{1}+s_{2}+s_{3} \geq \frac{d}{2}$ if $s_{i} \neq \frac{d}{2}, i=1,2,3$ or $s_{1}+s_{2}+s_{3}>\frac{d}{2}$ if $s_{i}=\frac{d}{2}$, for at least one $i$ then from [15, Prop. 6.1, (6.10)] we have that the following estimate holds for $\left(w_{1}, w_{2}, w_{3}\right) \in \mathbf{H}^{s_{1}}(\Omega) \times \mathbf{H}^{1+s_{2}}(\Omega) \times \mathbf{H}^{s_{3}}(\Omega)$ :

$$
\int_{\Omega}\left(w_{1} \cdot \nabla\right) w_{2} \cdot w_{3} d x \leq C\left\|w_{1}\right\|_{\mathbf{H}^{s_{1}}(\Omega)}\left\|w_{2}\right\|_{\mathbf{H}^{1+s_{2}}(\Omega)}\left\|w_{3}\right\|_{\mathbf{H}^{s_{3}}(\Omega)}
$$

Then we deduce that the nonlinear map $w \mapsto(w \cdot \nabla) w$ obeys the following boundedness and Lipschitz property: if $s \in\left[\frac{d-2}{2}, 1\right]$ then for $w, w_{1}, w_{2}$ in $\mathbf{V}^{s+1}(\Omega)$ we have

$$
\begin{aligned}
\|(w \cdot \nabla) w\|_{\mathbf{H}^{s-1}(\Omega)} \leq & C\|w\|_{\mathbf{V}^{s}(\Omega)}\|w\|_{\mathbf{V}^{s+1}(\Omega)} \\
\left\|\left(w_{1} \cdot \nabla\right) w_{1}-\left(w_{2} \cdot \nabla\right) w_{2}\right\|_{\mathbf{H}^{s-1}(\Omega)} \leq & C\left(\left\|w_{1}-w_{2}\right\|_{\mathbf{V}^{s}(\Omega)}\left(\left\|w_{1}\right\|_{\mathbf{V}^{s+1}(\Omega)}+\left\|w_{2}\right\|_{\mathbf{V}^{s+1}(\Omega)}\right)\right. \\
& \left.+\left\|w_{1}-w_{2}\right\|_{\mathbf{V}^{s+1}(\Omega)}\left(\left\|w_{1}\right\|_{\mathbf{V}^{s}(\Omega)}+\left\|w_{2}\right\|_{\mathbf{V}^{s}(\Omega)}\right)\right)
\end{aligned}
$$

Notice that to obtain (102)-(103) in the limit case $d=2$ and $s=0$, one must combine the antisymmetry property $\int_{\Omega}\left(w_{1} \cdot \nabla\right) w_{2} \cdot w_{3} d x=-\int_{\Omega}\left(w_{1} \cdot \nabla\right) w_{3} \cdot w_{2} d x$ for $\left(w_{1}, w_{2}, w_{3}\right) \in \mathbf{V}^{1}(\Omega) \times \mathbf{V}^{1}(\Omega) \times \mathbf{H}_{0}^{1}(\Omega)$ with $(101)$ for $\left(s_{1}, s_{2}, s_{3}\right)=(1 / 2,0,1 / 2)$ and the interpolation inequality $\|\cdot\|_{\mathbf{V}^{\frac{1}{2}(\Omega)}} \leq C\|\cdot\|_{\mathbf{V}^{0}(\Omega)}^{\frac{1}{2}}\|\cdot\|_{\mathbf{V}^{1}(\Omega)}^{\frac{1}{2}}$.

For an initial datum $w_{0} \in \mathbf{V}_{n}^{0}(\Omega)$ it is well known that (100) is equivalent to the following dynamical system in $\mathbf{V}_{n}^{0}(\Omega)$ :

$$
w^{\prime}=A w+B_{\omega} h-\kappa P(w \cdot \nabla) w \in\left[\mathcal{D}\left(A^{*}\right)\right]^{\prime}, \quad w(0)=w_{0},
$$

where $B_{\omega}$ is the bounded linear mapping defined from $\mathbf{L}^{2}(\Omega)$ into $\mathbf{V}_{n}^{0}(\Omega)$ by

$$
B_{\omega} h \stackrel{\text { def }}{=} P \mathbb{1}_{\omega} h
$$

Notice that its adjoint $B_{\omega}^{*} \in \mathcal{L}\left(\mathbf{V}_{n}^{0}(\Omega), \mathbf{L}^{2}(\Omega)\right)$ is simply given by $B_{\omega}^{*} y=\mathbb{1}_{\omega} y$ for all $y \in \mathbf{V}_{n}^{0}(\Omega)$. The following proposition, which is taken from [1, Prop. 1], gives a precise statement of the equivalence between formulations (104) and (100). For it, we need to define spaces of pressures with free mean:

$$
L_{\sharp}^{2}(\Omega) \stackrel{\text { def }}{=}\left\{p \in L^{2}(\Omega) \mid \int_{\Omega} p(x) d x=0\right\} \quad \text { and } \quad H_{\sharp}^{s}(\Omega) \stackrel{\text { def }}{=} H^{s}(\Omega) \cap L_{\sharp}^{2}(\Omega), \quad s \geq 0 .
$$

Proposition 21. Let $\kappa \in\{0,1\}, s \in[0,1]$ and assume that $h \in L_{\text {loc }}^{2}\left(\mathbf{L}^{2}(\Omega)\right)$.

1. If $\left.w \in W_{\text {loc }}\left(\mathbf{V}_{0}^{s+1} \Omega\right), \mathbf{V}_{0}^{s-1}(\Omega)\right)$ obeys $(104)$ then there exists a unique pressure function $p \in H_{l o c}^{-\frac{1}{2}+\frac{s}{2}}\left(H_{\sharp}^{s}(\Omega)\right)$ such that $(w, p)$ satisfies $(100)$.

2. Conversely, if $\left.(w, p) \in W_{l o c}\left(\mathbf{V}_{0}^{s+1} \Omega\right), \mathbf{V}_{0}^{s-1}(\Omega)\right) \times H_{l o c}^{-\frac{1}{2}+\frac{s}{2}}\left(H_{\sharp}^{s}(\Omega)\right)$ obeys $(100)$, then $w$ satisfies (104).

Next, to deal with a boundary control which is locally supported in an open subset of $\partial \Omega$ we follow the idea of [32]: we introduce a weight function $m \in C^{2}(\partial \Omega)$ with values in $[0,1]$, with support in $\Gamma_{m} \subset \partial \Omega$ and equal to 1 in $\Gamma_{1}$, where $\Gamma_{1}$ is an open nonempty subset of $\Gamma_{m}$, and we define a localization operator as follows:

$$
M(v)(x) \stackrel{\text { def }}{=} m(x) v(x)-\frac{m(x)}{\int_{\partial \Omega} m d \Gamma}\left(\int_{\partial \Omega} m v \cdot n d \Gamma\right) n(x)
$$

We observe that $M$ is self-adjoint and that $m \in C^{2}(\partial \Omega)$ ensures that for all $s \in[0,2]$ :

$$
M \in \mathcal{L}\left(\mathbf{V}^{s}(\partial \Omega)\right)
$$

Remark 22. Theorem 20 and Lemma 23 only require the assumptions that $\Omega$ is of class $C^{1,1}$ and $w_{S} \in \mathbf{V}^{1}(\Omega)$. The stronger assumption that $\Omega$ is of class $C^{2,1}$ is required to have (96) and (107) for $s \in[1,2]$. In particular, it implies that $n \in \mathbf{V}^{2}(\partial \Omega)$ in (107). The stronger assumption $w_{S} \in \mathbf{V}^{2}(\Omega)$ is needed later on, see Remark 29. 
We then consider Oseen $(\kappa=0)$ or Navier-Stokes $(\kappa=1)$ system with boundary condition localized on $\Gamma_{m}$ :

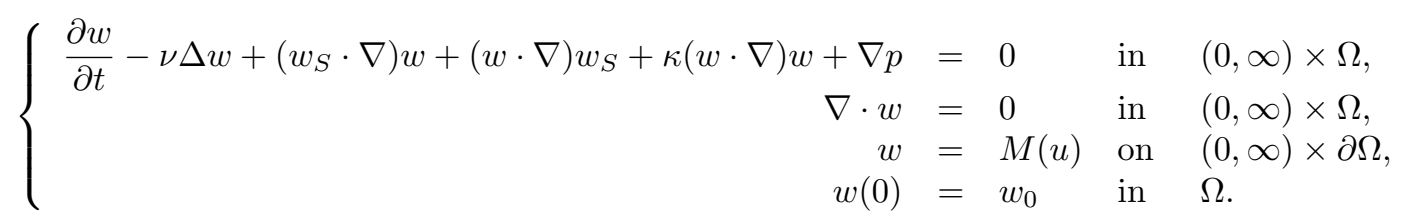

The notion of solution for (108) is the same as for (100). Next, to rewrite system (108) as an abstract dynamical system we first need to introduce the Dirichlet operator $D: \mathbf{V}^{0}(\partial \Omega) \rightarrow \mathbf{V}^{0}(\Omega)$ defined as follows: for $v \in \mathbf{V}^{0}(\partial \Omega)$, $w_{v}=D v$ is the unique solution of

$$
\begin{aligned}
& \lambda_{0} w_{v}-\nu \Delta w_{v}+\left(w_{v} \cdot \nabla\right) w_{S}+\left(w_{S} \cdot \nabla\right) w_{v}+\nabla q_{v}=0 \text { in } \Omega, \\
& \nabla \cdot w_{v}=0 \text { in } \Omega \\
& w_{v}=v \text { on } \partial \Omega \text {. }
\end{aligned}
$$

The operator $D$ defined as above satisfies the following properties, see [34].

Lemma 23. The following results hold.

- The operator $D$ is bounded from $\mathbf{V}^{s}(\partial \Omega)$ onto $\mathbf{V}^{s+\frac{1}{2}}(\Omega)$ for all $s \in[0,3 / 2]$.

- The adjoint of $D$ is defined by

$$
D^{*} f=\chi n-\nu \frac{\partial \varepsilon}{\partial n} \quad \text { for all } \in \mathbf{V}^{0}(\Omega),
$$

where $(\varepsilon, \chi) \in \mathbf{V}_{0}^{2}(\Omega) \times H^{1}(\Omega)$ is the solution of

$$
\begin{aligned}
& \lambda_{0} \varepsilon-\nu \Delta \varepsilon+{ }^{t}\left(\nabla w_{S}\right) \varepsilon-\left(w_{S} \cdot \nabla\right) \varepsilon+\nabla \chi=f \text { in } \Omega, \\
& \nabla \cdot \varepsilon=0 \text { in } \Omega \text {, } \\
& \varepsilon=0 \text { on } \partial \Omega \text {, } \\
& \int_{\partial \Omega} \chi d \Gamma=0 .
\end{aligned}
$$

Thus, we introduce the linear control operator:

$$
B_{\Gamma_{m}}: \mathbf{V}^{0}(\partial \Omega) \rightarrow\left[\mathcal{D}\left(A^{*}\right)\right]^{\prime}, \quad v \mapsto\left(\lambda_{0}-A\right) P D M(v) .
$$

In the above setting $\left[\mathcal{D}\left(A^{*}\right)\right]^{\prime}$ is the dual space of $\mathcal{D}\left(A^{*}\right)$ with respect to the pivot space $\mathbf{V}_{n}^{0}(\Omega)$. It corresponds to the space $X_{-1}$ in the terminology of [39]. Notice that for $3 / 4<\gamma<1$, by combining (107) with $s=0$, the first point of Lemma 23 with $s=0$, (96) with $s=2(1-\gamma)$ and (99) with $\theta=1-\gamma$, we obtain $P D M \in$ $\mathcal{L}\left(\mathbf{V}^{0}(\partial \Omega), \mathcal{D}\left(\left(\lambda_{0}-A\right)^{1-\gamma}\right)\right.$, which means that $B_{\Gamma_{m}}$ is strictly relatively bounded with respect to $\lambda_{0}-A$ :

$$
\left(\lambda_{0}-A\right)^{-\gamma} B_{\Gamma_{m}}=\left(\lambda_{0}-A\right)^{1-\gamma} P D M \in \mathcal{L}\left(\mathbf{V}^{0}(\partial \Omega), \mathbf{V}_{n}^{0}(\Omega)\right) \quad \text { for } 3 / 4<\gamma<1 .
$$

According to [34] system (108) can be written under the form

$$
\begin{aligned}
P w^{\prime} & =A P w+B_{\Gamma_{m}} u-\kappa P(w \cdot \nabla) w \in\left[\mathcal{D}\left(A^{*}\right)\right]^{\prime}, \quad w(0)=w_{0}, \\
(I-P) w & =(I-P) D M(u),
\end{aligned}
$$

which implies that (108) can be reduced to a dynamical system satisfied by $y=P w$ and which involves a nonlinear term depending on both variable $y$ and $u$ :

$$
y^{\prime}=A y+B_{\Gamma_{m}} u-\kappa P((y+(I-P) D M(u)) \cdot \nabla)(y+(I-P) D M(u)), \quad y(0)=P w_{0} .
$$

The following proposition, which is taken from [3, Prop. 4], gives a precise statement of the equivalence between formulations (111)-(112) and (108).

Proposition 24. Let $\kappa \in\{0,1\}$ and assume that $u \in L_{l o c}^{2}\left(\mathbf{V}^{s+\frac{1}{2}}(\partial \Omega)\right)$ with $s \in[0,1]$.

1. If $\left.w \in L_{\text {loc }}^{2}\left(\mathbf{V}^{s+1} \Omega\right)\right) \cap H_{\text {loc }}^{\frac{s}{2}+\frac{1}{2}}\left(\mathbf{V}^{0}(\Omega)\right)$ obeys (111)-(112) then there exists a unique pressure function $p \in H_{l o c}^{-\frac{1}{2}+\frac{s}{2}}\left(H_{\sharp}^{s}(\Omega)\right)$ such that $(w, p)$ satisfies $(108)$.

2. Conversely, if $\left.(w, p) \in\left[L_{\text {loc }}^{2}\left(\mathbf{V}^{s+1} \Omega\right)\right) \cap H_{l o c}^{\frac{s}{2}+\frac{1}{2}}\left(\mathbf{V}^{0}(\Omega)\right)\right] \times H_{l o c}^{-\frac{1}{2}+\frac{s}{2}}\left(H_{\sharp}^{s}(\Omega)\right)$ obeys (108), then $w$ satisfies (111)-(112). 


\subsection{Stabilization of the Navier-Stokes system by means of a finite dimensional internal control}

Here, we come back to the question of stabilizing the solutions of the Navier-Stokes system $(100)_{\kappa=1}$. At a first step, we consider the Oseen system $(100)_{\kappa=0}$ that we have rewritten as

$$
w^{\prime}=A w+B h \in\left[\mathcal{D}\left(A^{*}\right)\right]^{\prime}, \quad w(0)=w_{0},
$$

where $A$ and $B \stackrel{\text { def }}{=} B_{\omega}$ are defined by $(97)$ and by (105). To fit the framework of Section 3 we set $\mathcal{X} \stackrel{\text { def }}{=} \mathbf{V}_{n}^{0}(\Omega)$ and $\mathcal{V} \stackrel{\text { def }}{=} \mathbf{L}^{2}(\Omega)$ and we recall that $A: \mathcal{D}(A) \subset \mathcal{X} \rightarrow \mathcal{X}$ and of $B: \mathcal{V} \rightarrow\left[\mathcal{D}\left(A^{*}\right)\right]^{\prime}$ must satisfy

(a) $A$ has a compact resolvent;

(b) $A$ is the generator of an analytic semigroup on $\mathcal{X}$;

(c) $\widehat{A}^{-\gamma} B: \mathcal{V} \rightarrow \mathcal{X}$ is bounded for some $0 \leq \gamma<1$;

(d) Conditions 1 and 2 of Corollary 4 .

Conditions (a) and (b) follow from Theorem 20 and Condition (c) is true for $\gamma=0$ since $B \stackrel{\text { def }}{=} B_{\omega}$ is bounded. Condition (d) is obtained by Theorem 6 by using the exact controllability result stated in [18] (see also [28] and $[26])$. According to the expression of $B^{*}=B_{\omega}^{*}$ given by (105), the unique continuation property which is obtain from [18] by Theorem 6 can be stated as follows:

Theorem 25. For all $\lambda \in \mathbb{C}$, every solution $(\varepsilon, \chi) \in \mathbf{H}^{2}(\Omega) \times H^{1}(\Omega)$ of

$$
\begin{aligned}
& \lambda \varepsilon-\nu \Delta \varepsilon-\left(w_{S} \cdot \nabla\right) \varepsilon+{ }^{t}\left(\nabla w_{S}\right) \varepsilon+\nabla \chi=0 \quad \text { in } \Omega, \quad \int_{\partial \Omega} \chi d \Gamma=0, \\
& \nabla \cdot \varepsilon=0 \text { in } \Omega \\
& \varepsilon=0 \text { on } \partial \Omega \text {, }
\end{aligned}
$$

satisfying $\varepsilon=0$ in $\omega$ is necessarily equal to zero.

Moreover, to fit the framework of Section 4 the following additional statements are required:

(e) For all $\theta \in[0,1]$ we have the equalities $\mathcal{D}\left(\widehat{A}^{\theta}\right)=[\mathcal{D}(A), \mathcal{X}]_{1-\theta}$ and $\mathcal{D}\left(\widehat{A}^{* \theta}\right)=\left[\mathcal{D}\left(A^{*}\right), \mathcal{X}\right]_{1-\theta}$.

(f) There is a Hilbert space $\mathcal{O}$ and an isomorphism mapping $G: \mathcal{X} \rightarrow \mathcal{O}$ such that $G^{*} G \in \mathcal{L}\left(\mathcal{D}\left(\widehat{A}^{\frac{1}{2}}\right), \mathcal{D}\left(\widehat{A}^{* \frac{1}{2}}\right)\right)$.

Condition (e) follows from (99) and since $\mathcal{D}\left(\widehat{A}^{\frac{1}{2}}\right)=\mathcal{D}\left(\widehat{A}^{* \frac{1}{2}}\right)$ Condition (f) can be obtained by choosing $G$ as the identity in $\mathbf{V}_{n}^{0}(\Omega)$. Next, let us recall the notations of Sections 3 and 4 .

- $\sigma>0$ is an expected rate of decrease, $\Sigma=\left\{\lambda_{k} \mid k \in \mathbb{N}^{*}\right\}$ is the spectrum of $A$ and:

$$
\cdots \leq \Re \lambda_{N+1}<-\sigma<\Re \lambda_{N} \leq \cdots \leq \Re \lambda_{2} \leq \Re \lambda_{1}
$$

- $\mathcal{X}_{N}=P_{N}\left(\mathbf{V}_{n}^{0}(\Omega)\right)$ where $P_{N}: \mathbf{V}_{n}^{0}(\Omega) \rightarrow \mathcal{X}_{N}$ is the projection operator related to $\lambda_{1}, \ldots, \lambda_{N}$ defined by:

$$
P_{N} \stackrel{\text { def }}{=}-\frac{1}{2 \pi \imath} \int_{\Gamma_{N}}(\xi-A)^{-1} d \xi,
$$

where $\Gamma_{N}$ is a positively-oriented curve obeying $\Gamma_{N}=\bar{\Gamma}_{N}$, and enclosing $\Sigma_{N}$ but no other point of $\Sigma$. We have $\bar{P}_{N}=P_{N}$ and $\mathcal{X}_{N}$ is a real finite dimensional subspace of $\mathbf{V}_{n}^{0}(\Omega)$.

- $\mathcal{X}_{N}^{*}=P_{N}^{*}\left(\mathbf{V}_{n}^{0}(\Omega)\right)$ where $P_{N}^{*}$ is the adjoint of $P_{N}$.

- $\left\{\varepsilon_{k}^{i}, i=1, \ldots, \ell_{k}\right\}$ a basis of $\operatorname{Ker}\left(A^{*}-\bar{\lambda}_{k}\right), \ell_{k}$ is the geometric multiplicity of $\bar{\lambda}_{k}$ and we set

$$
\mathcal{F}_{N}=\operatorname{span}_{\mathbb{R}}\left\{\Re \varepsilon_{k}^{i}, \Im \varepsilon_{k}^{i}, k=1, \ldots, N, i=1, \ldots \ell_{k}\right\} .
$$

- $K \geq \ell_{k}$ for all $k=1, \ldots, N,\left(v_{1}, \ldots, v_{K}\right) \in B^{*}\left(\mathcal{F}_{N}\right)^{K}$ is the family given by Theorem 7 .

- $\Pi \in \mathcal{L}\left(\mathcal{X}_{N}, \mathcal{X}_{N}^{*}\right)$ is the Riccati operator solution to $(36),(37)$. 
- $V \in \mathcal{L}\left(\mathbb{R}^{K},\left[\mathcal{D}\left(A^{*}\right)\right]^{\prime}\right)$ is defined by (33) and $A_{\Pi}$ is the closed-loop operator defined by (59).

Notice that the expression $B^{*}=B_{\omega}^{*} y=\mathbb{1}_{\omega} y$ ensures that $v_{j}, j=1, \ldots K$ are supported in $\omega$. Moreover, according to Proposition 21, system (57) with $y=w$ can be rewritten as follows:

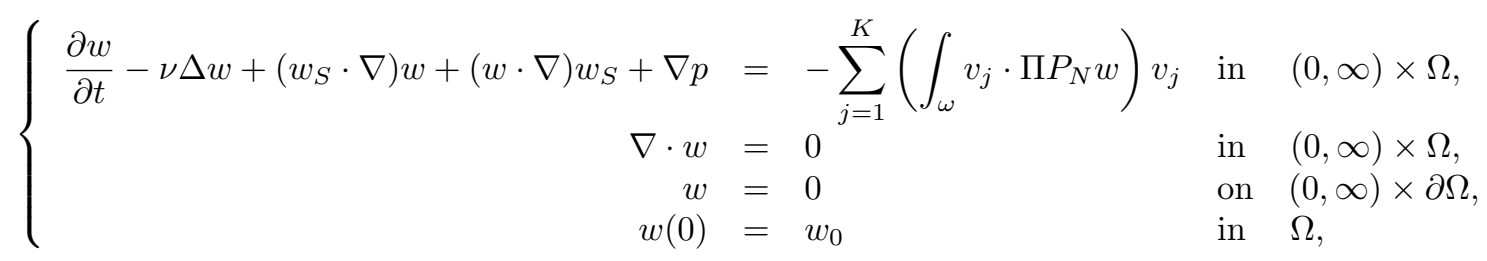

Notice also that here $B \stackrel{\text { def }}{=} B_{\omega}$ is a bounded operator from $\mathcal{V} \stackrel{\text { def }}{=} \mathbf{L}^{2}(\Omega)$ into $\mathcal{X} \stackrel{\text { def }}{=} \mathbf{V}_{n}^{0}(\Omega)$ and then $V \in \mathcal{L}\left(\mathbb{R}^{K}, \mathcal{X}\right)$. As a consequence, $A_{\Pi}$ is a bounded perturbation of $A$, their domain coincide and we have:

$$
\mathcal{X}^{s} \stackrel{\text { def }}{=} \mathcal{D}\left(A_{\Pi}^{\frac{s}{2}}\right)=\mathbf{V}_{0}^{s}(\Omega), \quad \forall s \in[0,2] .
$$

Then we deduce that the solution of (116) is exponentially stable, more precisely:

Proposition 26. Let $s \in[0,1]$ and $w_{0} \in \mathbf{V}_{0}^{s}(\Omega)$. Then (116) admits a unique solution

$$
(w, p) \in W\left(\mathbf{V}_{0}^{1+s}(\Omega), \mathbf{V}_{0}^{s-1}(\Omega)\right) \times H^{-\frac{1}{2}+\frac{s}{2}}\left(H_{\sharp}^{s}(\Omega)\right)
$$

which obeys:

$$
\|w\|_{\mathbf{V}_{0}^{s}(\Omega)} \leq C\left\|w_{0}\right\|_{\mathbf{V}_{0}^{s}(\Omega)} e^{-\sigma t} \quad \text { for all } t \geq 0 .
$$

Proof. A direct consequence of Proposition 10 is that system (57) is exponentially stable in the norm of $\mathcal{D}\left(A_{\Pi}^{\frac{s}{2}}\right)$, and then the conclusion follows from (117) and Proposition 21.

Finally, we deduce that the solution of the nonlinear system

$$
\left\{\begin{aligned}
\frac{\partial w}{\partial t}-\nu \Delta w+\left(w_{S} \cdot \nabla\right) w+(w \cdot \nabla) w_{S}+(w \cdot \nabla) w+\nabla p & =-\sum_{j=1}^{K}\left(\int_{\omega} v_{j} \cdot \Pi P_{N} w\right) v_{j} & \text { in } & (0, \infty) \times \Omega, \\
\nabla \cdot w & =0 & & \text { in } \quad(0, \infty) \times \Omega, \\
w & =0 & & \text { on } \quad(0, \infty) \times \partial \Omega, \\
w(0) & =w_{0} & & \text { in } \Omega,
\end{aligned}\right.
$$

is locally exponentially stable, more precisely:

Theorem 27. Let $s \in\left[\frac{d-2}{2}, 1\right]$ and $w_{0} \in \mathbf{V}_{0}^{s}(\Omega)$. There exists $\mu>0$ such that if $\left\|w_{0}\right\|_{\mathbf{V}_{0}^{s}(\Omega)} \leq \mu$ then (119) admits a solution $(w, p) \in W\left(\mathbf{V}_{0}^{1+s}(\Omega), \mathbf{V}_{0}^{s-1}(\Omega)\right) \times H^{-\frac{1}{2}+\frac{s}{2}}\left(H_{\sharp}^{s}(\Omega)\right)$, which is unique within the class of function of $\left(L_{l o c}^{2}\left(\mathbf{V}_{0}^{s+1}(\Omega)\right) \cap L_{l o c}^{\infty}\left(\mathbf{V}_{0}^{s}(\Omega)\right)\right) \times H_{l o c}^{-\frac{1}{2}+\frac{s}{2}}\left(H_{\sharp}^{s}(\Omega)\right)$, and which obeys:

$$
\|w\|_{\mathbf{V}_{0}^{s}(\Omega)} \leq C\left\|w_{0}\right\|_{\mathbf{V}_{0}^{s}(\Omega)} e^{-\sigma t} \quad \text { for all } t \geq 0 .
$$

Proof. The conclusion follows by combining (117), Theorem 15 and Proposition 24. Notice that assumption (72)-(73) required for $N(\xi) \stackrel{\text { def }}{=}-P(\xi \cdot \nabla) \xi$ to apply Theorem 15 is obtained from (102)-(103).

\subsection{Stabilization of the Navier-Stokes system by means of a finite dimensional boundary control}

Here, we come back to the question of stabilizing the solutions of the Navier-Stokes system $(108)_{\kappa=1}$ by means of a finite dimensional boundary feedback control. At a first step, we consider the Oseen system $(108)_{\kappa=0}$ that we have rewritten as

$$
y^{\prime}=A y+B u \in\left[\mathcal{D}\left(A^{*}\right)\right]^{\prime}, \quad y(0)=P w_{0},
$$

where $A$ and $B \stackrel{\text { def }}{=} B_{\Gamma_{m}}$ are defined by $(97)$ and by (109) and where $y=P w$. Here, we are in the framework of Section 3 with $\mathcal{X} \stackrel{\text { def }}{=} \mathbf{V}_{n}^{0}(\Omega)$ and $\mathcal{V} \stackrel{\text { def }}{=} \mathbf{V}^{0}(\partial \Omega)$ : conditions (a), (b), (e) and (f) have been checked in the 
previous section, condition (c) is given by (110) and condition (d) is obtained by Theorem 6 by using the exact controllability result stated in [18] (see also [28] and [26]). Indeed, although the previous cited work only deals with a distributed control localized in an open part of the domain, the controllability of $(108)_{\kappa=0}$ is obtained with a classical extension procedure (see for instance the appendix of [2]). Notice that from [3, Prop. 3] we deduce that the adjoint of $B$ is given by

$$
B^{*} \varepsilon=m\left(\chi n-\nu \frac{\partial \varepsilon}{\partial n}\right) \quad \forall \varepsilon \in \mathbf{V}_{0}^{2}(\Omega)
$$

where $\chi=\chi(\varepsilon) \in H^{1}(\Omega)$ is a pressure function solution to $\nabla \chi=(I-P)\left(\nu \Delta \varepsilon-{ }^{t}\left(\nabla w_{S}\right) \varepsilon+\left(w_{S} \cdot \nabla\right) \varepsilon\right)$ and $\int_{\partial \Omega} m \chi d \Gamma=0$, which also means that:

$$
\left\{\begin{array}{l}
\Delta \chi=\nabla \cdot\left(\left(w_{S} \cdot \nabla\right) \varepsilon-{ }^{t}\left(\nabla w_{S}\right) \varepsilon\right) \text { in } \Omega, \quad \int_{\partial \Omega} m \chi d \Gamma=0 \\
\frac{\partial \chi}{\partial n}=\left(\nu \Delta \varepsilon+\left(w_{S} \cdot \nabla\right) \varepsilon-{ }^{t}\left(\nabla w_{S}\right) \varepsilon\right) \cdot n \text { on } \partial \Omega
\end{array}\right.
$$

In particular, if $\varepsilon \in \operatorname{Ker}\left(A^{*}-\lambda\right)$ then $B^{*} \varepsilon=m\left(\chi n-\nu \frac{\partial \varepsilon}{\partial n}\right)$ where $(\varepsilon, \chi)$ satisfies:

$$
\begin{aligned}
& \lambda \varepsilon-\nu \Delta \varepsilon-\left(w_{S} \cdot \nabla\right) \varepsilon+{ }^{t}\left(\nabla w_{S}\right) \varepsilon+\nabla \chi=0 \quad \text { in } \Omega, \quad \int_{\partial \Omega} m \chi d \Gamma=0, \\
& \nabla \cdot \varepsilon=0 \text { in } \Omega \\
& \varepsilon=0 \text { on } \partial \Omega \text {, }
\end{aligned}
$$

and the unique continuation property which is obtain from [18] by Theorem 6 can be stated as follows:

Theorem 28. For all $\lambda \in \mathbb{C}$, every solution $(\varepsilon, \chi) \in \mathbf{H}^{2}(\Omega) \times H^{1}(\Omega)$ of (124) satisfying

$$
m\left(\chi n-\nu \frac{\partial \varepsilon}{\partial n}\right)=0 \quad \text { on } \partial \Omega
$$

is necessarily equal to zero.

Notice that now, the family $\left(v_{1}, \ldots, v_{K}\right) \in B^{*}\left(\mathcal{F}_{N}\right)^{K}$ given by Theorem 7 belongs to $\mathbf{V}^{0}(\partial \Omega)$. More precisely, the functions $v_{j}, j=1, \ldots, K$ are supported in $\Gamma_{m}$ (the support of $m$ ) and since $\Omega$ is of class $C^{2,1}$ and $w_{S} \in \mathbf{V}^{2}(\Omega)$ we have $B^{*}\left(\mathcal{F}_{N}\right) \subset \mathbf{V}^{\frac{3}{2}}(\partial \Omega)$ and $\left(v_{1}, \ldots, v_{K}\right) \subset\left(\mathbf{V}^{\frac{3}{2}}(\partial \Omega)\right)^{K}$.

Remark 29. (i) The assumption $w_{S} \in \mathbf{V}^{2}(\Omega)$ is only required for the inclusion $\mathcal{F}_{N} \subset \mathbf{V}^{\frac{3}{2}}(\partial \Omega)$. Indeed, suppose that $\varepsilon \in \operatorname{Ker}\left(A^{*}-\lambda\right)$. Then $\varepsilon$ belongs to $\mathcal{D}\left(A^{*}\right) \subset \mathbf{H}^{2}(\Omega)$ and with $w_{S} \in \mathbf{H}^{2}(\Omega)$ and Sobolev embeddings we obtain $\left(w_{S} \cdot \nabla\right) \varepsilon+{ }^{t}\left(\nabla w_{S}\right) \varepsilon \in \mathbf{H}^{1}(\Omega)$. As a consequence, since $\varepsilon$ satisfies (124) and since $\Omega$ is of class $C^{2,1}$, regularity result for Stokes system [10, Thm. III.3.16 p.148] yields $(\varepsilon, \chi(\varepsilon)) \in \mathbf{H}^{3}(\Omega) \times H^{2}(\Omega)$ and $B^{*} \varepsilon=m\left(\chi(\varepsilon) n-\nu \frac{\partial \varepsilon}{\partial n}\right) \in \mathbf{V}^{\frac{3}{2}}(\partial \Omega)$. In fact, with an analogue local argument one can prove that it is sufficient to have $w_{S} \in \mathbf{V}^{1}(\Omega) \cap \mathbf{H}^{2}(\mathcal{O})$ where $\mathcal{O}$ is a neighborhood of the part of the boundary where the control is acting: $\Gamma_{m} \subset \partial \Omega \cap \partial \mathcal{O}$.

(ii) Since the assumption that $\Omega$ of class $C^{2,1}$ also ensures that (96), (107) are true for $s=2$ we have:

$$
\operatorname{PDM}\left(v_{j}\right) \in \mathbf{V}_{n}^{2}(\Omega) \quad \forall j=1, \ldots, K .
$$

Proposition 30. For all $s \in[0,2]$ the following characterization holds:

$$
\begin{aligned}
& \mathcal{X}^{s} \stackrel{\text { def }}{=} \mathcal{D}\left(A_{\Pi}^{\frac{s}{2}}\right)=\left\{\xi \in \mathbf{V}_{n}^{s}(\Omega) \mid \xi+P D \sum_{j=1}^{K}\left(\int_{\partial \Omega} v_{j} \cdot\left(B^{*} \Pi\right) P_{N} \xi d \Gamma\right) M\left(v_{j}\right) \in \mathbf{V}_{0}^{s}(\Omega)\right\}, \\
& \mathcal{X}^{-s} \stackrel{\text { def }}{=}\left[\mathcal{D}\left(A_{\Pi}^{* \frac{s}{2}}\right)\right]^{\prime}=\mathbf{V}_{0}^{-s}(\Omega) .
\end{aligned}
$$

Proof. Without loss of generality we can assume that the values $\lambda_{0}$ in (98) and in Section 4 are the same. Then from (65) with (99), and with the expression of $V$ and of $B$ given by (33) and (109), we first deduce that:

$$
\mathcal{D}\left(A_{\Pi}^{\frac{s}{2}}\right)=\left\{\xi \in \mathbf{V}_{n}^{0}(\Omega) \mid \xi+\sum_{j=1}^{K}\left(\int_{\partial \Omega} v_{j} \cdot\left(B^{*} \Pi\right) P_{N} \xi d \Gamma\right) P D M\left(v_{j}\right) \in \mathbf{V}_{0}^{s}(\Omega)\right\} .
$$

Thus with (125) we deduce that $\mathcal{D}\left(A_{\Pi}^{\frac{s}{2}}\right)$ is the closed subspace of $\mathbf{V}_{n}^{s}(\Omega)$ defined by (126). Finally, (127) is a direct consequence of the second equality in (65) with (99). 
Corollary 31. For $s \in[0,2]$, let us set

$\mathbf{V}_{\Pi}^{s}(\Omega)=\mathbf{V}^{s}(\Omega) \quad$ if $\quad s<\frac{1}{2}$,

$\mathbf{V}_{\Pi}^{\frac{1}{2}}(\Omega)=\left\{y \in \mathbf{V}^{\frac{1}{2}}(\Omega) \mid \int_{\Omega} \operatorname{dist}(x, \partial \Omega)^{-1}\left(y(x)+D\left[\sum_{j=1}^{K}\left(\int_{\partial \Omega} v_{j} \cdot\left(B^{*} \Pi\right) P_{N} P y d \Gamma\right) M\left(v_{j}\right)\right](x)\right) d x<+\infty\right\}$,

$\mathbf{V}_{\Pi}^{s}(\Omega)=\left\{y \in \mathbf{V}^{s}(\Omega) \mid y+\sum_{j=1}^{K}\left(\int_{\partial \Omega} v_{j} \cdot\left(B^{*} \Pi\right) P_{N} P y d \Gamma\right) M\left(v_{j}\right)=0 \quad\right.$ on $\left.\partial \Omega\right\} \quad$ if $\quad s>\frac{1}{2}$.

Then $P \mathbf{V}_{\Pi}^{s}(\Omega)=\mathcal{X}^{s}$.

Using Proposition 10 the solution of the system

$$
\left\{\begin{aligned}
\frac{\partial w}{\partial t}-\nu \Delta w+\left(w_{S} \cdot \nabla\right) w+(w \cdot \nabla) w_{S}+\nabla p & =0 & & \text { in }(0, \infty) \times \Omega, \\
\nabla \cdot w & =0 & & \text { in } \quad(0, \infty) \times \Omega, \\
w & =-\sum_{j=1}^{K}\left(\int_{\partial \Omega} v_{j} \cdot\left(B^{*} \Pi\right) P_{N} P w d \Gamma\right) M\left(v_{j}\right) & & \text { on }(0, \infty) \times \partial \Omega, \\
w(0) & =w_{0} & & \text { in } \Omega,
\end{aligned}\right.
$$

is exponentially stable, more precisely:

Proposition 32. Let $s \in[0,1]$ and $w_{0} \in \mathbf{V}_{\Pi}^{s}(\Omega)$. Then (128) admits a unique solution

$$
(w, p) \in W\left(\mathbf{V}^{1+s}(\Omega), \mathbf{V}_{0}^{s-1}(\Omega)\right) \times H^{-\frac{1}{2}+\frac{s}{2}}\left(H_{\sharp}^{s}(\Omega)\right)
$$

which obeys:

$$
\|w\|_{\mathbf{V}^{s}(\Omega)} \leq C\left\|P w_{0}\right\|_{\mathbf{V}^{s}(\Omega)} e^{-\sigma t} \quad \text { for all } t \geq 0 .
$$

Proof. Since $W\left(\mathbf{V}^{1+s}(\Omega), \mathbf{V}_{0}^{s-1}(\Omega)\right) \hookrightarrow L^{2}\left(\mathbf{V}^{s+1}(\Omega)\right) \cap H^{\frac{s}{2}+\frac{1}{2}}\left(\mathbf{V}^{0}(\Omega)\right)$, then by Proposition 24 it suffices to prove that system $(111)_{\kappa=0},(112)$ with $u=-\sum_{j=1}^{K}\left(\int_{\partial \Omega} v_{j} \cdot\left(B^{*} \Pi\right) P_{N} P w d \Gamma\right) v_{j}$ admits a unique solution in $W\left(\mathbf{V}^{1+s}(\Omega), \mathbf{V}_{0}^{s-1}(\Omega)\right)$ which obeys (129). Let us define $J \in \mathcal{L}\left(\mathbf{V}_{n}^{0}(\Omega), \mathbf{V}^{0}(\Omega)\right)$ by:

$$
J(y) \stackrel{\text { def }}{=}-\sum_{i=1}^{K}\left(\int_{\partial \Omega} v_{j} \cdot\left(B^{*} \Pi\right) P_{N} P y d \Gamma\right)(I-P) D M\left(v_{i}\right) .
$$

From $P_{N} \in \mathcal{L}\left(\mathbf{V}_{0}^{s-1}(\Omega), \mathbf{V}_{0}^{2}(\Omega)\right)$ and from $(125)$ we deduce that $J \in \mathcal{L}\left(\mathbf{V}_{0}^{s-1}(\Omega), \mathbf{V}^{2}(\Omega)\right)$ and then:

$$
J \in \mathcal{L}\left(W\left(\mathcal{X}^{s+1}, \mathcal{X}^{s-1}\right), H^{1}\left(\mathbf{V}^{2}(\Omega)\right) .\right.
$$

Thus, we verify that $y \in W\left(\mathcal{X}^{s+1}, \mathcal{X}^{s-1}\right)$ obeys:

$$
y^{\prime}+A_{\Pi} y=0, \quad y(0)=P w_{0},
$$

if and only if, $w=y+J(y) \in W\left(\mathbf{V}^{1+s}(\Omega), \mathbf{V}_{0}^{s-1}(\Omega)\right)$ obeys $(111)_{\kappa=0},(112)$ with the control function $u=-\sum_{j=1}^{K}\left(\int_{\partial \Omega} v_{j} \cdot\left(B^{*} \Pi\right) P_{N} P w d \Gamma\right) M\left(v_{j}\right)$. In particular $w_{0} \in \mathbf{V}_{\Pi}^{s}(\Omega)$ implies $w(0)=P w_{0}+J\left(P w_{0}\right)=w_{0}$. Then since by Corollary 31 we have $P w_{0} \in \mathcal{X}^{s}$, then from Proposition 10 with maximal regularity result for analytic semigroup (8) we deduce the existence of a unique $y \in W\left(\mathcal{X}^{s+1}, \mathcal{X}^{s-1}\right)$ solution of (131), and the conclusion follows. Notice that (129) is an easy consequence of (60).

Using Theorem 15, the solution of the nonlinear system

$$
\left\{\begin{aligned}
\frac{\partial w}{\partial t}-\nu \Delta w+\left(w_{S} \cdot \nabla\right) w+(w \cdot \nabla) w_{S}+\nabla p & =-(w \cdot \nabla) w & & \text { in }(0, \infty) \times \Omega, \\
\nabla \cdot w & =0 & & \text { in } \quad(0, \infty) \times \Omega, \\
w & =-\sum_{j=1}^{K}\left(\int_{\partial \Omega} v_{j} \cdot\left(B^{*} \Pi\right) P_{N} P w d \Gamma\right) M\left(v_{j}\right) & & \text { on }(0, \infty) \times \partial \Omega, \\
w(0) & =w_{0} & & \text { in } \Omega,
\end{aligned}\right.
$$

is locally exponentially stable, more precisely: 
Theorem 33. Let $s \in\left[\frac{d-2}{2}, 1\right]$ and $w_{0} \in \mathbf{V}_{\Pi}^{s}(\Omega)$. There exists $\mu>0$ such that if $\left\|P w_{0}\right\|_{\mathbf{V}_{n}^{s}(\Omega)} \leq \mu$ then (132) admits a solution $(w, p) \in W\left(\mathbf{V}^{1+s}(\Omega), \mathbf{V}_{0}^{s-1}(\Omega)\right) \times H^{-\frac{1}{2}+\frac{s}{2}}\left(H_{\sharp}^{s}(\Omega)\right)$, which is unique within the class of function of $\left(L_{l o c}^{2}\left(\mathbf{V}^{s+1}(\Omega)\right) \cap L_{l o c}^{\infty}\left(\mathbf{V}^{s}(\Omega)\right)\right) \times H_{l o c}^{-\frac{1}{2}+\frac{s}{2}}\left(H_{\sharp}^{s}(\Omega)\right)$, and which obeys:

$$
\|w\|_{\mathbf{V}^{s}(\Omega)} \leq C\left\|P w_{0}\right\|_{\mathbf{V}^{s}(\Omega)} e^{-\sigma t} \quad \text { for all } t \geq 0
$$

Proof. Since $W\left(\mathbf{V}^{1+s}(\Omega), \mathbf{V}_{0}^{s-1}(\Omega)\right) \hookrightarrow L^{2}\left(\mathbf{V}^{s+1}(\Omega)\right) \cap H^{\frac{s}{2}+\frac{1}{2}}\left(\mathbf{V}^{0}(\Omega)\right)$, then by Proposition 24 it suffices to prove that there is $\mu>0$ such that for $\left\|P w_{0}\right\|_{\mathbf{V}_{n}^{s}(\Omega)} \leq \mu$ system $(111)_{\kappa=1},(112)$ with $u=-\sum_{j=1}^{K}\left(\int_{\partial \Omega} v_{j} \cdot\left(B^{*} \Pi\right) P_{N} P w d \Gamma\right) v_{j}$ admits a solution in $W\left(\mathbf{V}^{1+s}(\Omega), \mathbf{V}_{0}^{s-1}(\Omega)\right)$ which is unique within the class of function of $L_{l o c}^{2}\left(\mathbf{V}^{s+1}(\Omega)\right) \cap$ $L_{l o c}^{\infty}\left(\mathbf{V}^{s}(\Omega)\right)$ and which obeys $(133)$. Thus, we set $N(y) \stackrel{\text { def }}{=}((y+J(y)) \cdot \nabla)(y+J(y))$ where $J$ is given by $(130)$ and, as in the proof of Proposition 32 , we verify that $y \in W\left(\mathcal{X}^{s+1}, \mathcal{X}^{s-1}\right)$ obeys:

$$
y^{\prime}+A_{\Pi} y=N(y), \quad y(0)=P w_{0},
$$

if and only if, $w=y+J(y)$ obeys $(111)_{\kappa=1},(112)$ with $u=-\sum_{j=1}^{K}\left(\int_{\partial \Omega} v_{j} \cdot\left(B^{*} \Pi\right) P_{N} P w d \Gamma\right) v_{j}$. Then by combining estimates (102),(103) and the fact that $J \in\left(\mathbf{V}_{n}^{s}(\Omega), \mathbf{V}^{s}(\Omega)\right)$ for all $s \in[0,2]$ we deduce that $N$ obeys (72),(73) and, since $w_{0} \in \mathbf{V}_{\Pi}^{s}(\Omega)$ implies $P w_{0} \in \mathcal{X}^{s}$, the conclusion follows from Theorem 15.

Let us now consider the question of stabilizing the Navier-Stokes system by means of a finite dimensional dynamical control. We first recall the additional notations introduced in Subsection 3.5:

- $\Pi_{2} \in \mathcal{L}\left(\mathcal{X}_{N}, \mathbb{R}^{K}\right)$ and $\Pi_{3} \in \mathcal{L}\left(\mathbb{R}^{K}\right)$ are defined by (49), (50) and (51).

- $D_{K}: \mathbb{R}^{K} \rightarrow \mathbb{R}^{K}$ and $G_{K}: \mathbb{R}^{K} \rightarrow \mathbb{R}^{K}$ are linear operators and $G_{K}$ is invertible.

- $\mathbb{A}_{\boldsymbol{\Pi}}$ is the closed-loop operator defined by (87).

Proposition 34. For all $s \in[0,2]$ the following characterization holds:

$$
\begin{aligned}
\mathcal{D}\left(\mathbb{A}_{\Pi}^{\frac{s}{2}}\right) & =\left\{Y={ }^{t}(y, \bar{u}) \in \mathbf{V}_{n}^{s}(\Omega) \times \mathbb{R}^{K} \mid y-P D \sum_{j=1}^{K} u_{j} M\left(v_{j}\right) \in \mathbf{V}_{0}^{s}(\Omega)\right\}, \\
\mathcal{D}\left(\mathbb{A}_{\Pi}^{* \frac{s}{2}}\right) & =\mathbf{V}_{0}^{s}(\Omega) \times \mathbb{R}^{K} .
\end{aligned}
$$

Proof. Since we have (125), then (134) follows from (90), (82), (99) by recalling (33), (109). Moreover, (135) follows from (90),(83) and (99).

Using Proposition 17 the solution of the system:

$$
\left\{\begin{array}{rlrl}
\frac{\partial w}{\partial t}-\nu \Delta w+\left(w_{S} \cdot \nabla\right) w+(w \cdot \nabla) w_{S}+\nabla p & =0 & & \text { in }(0, \infty) \times \Omega, \\
\nabla \cdot w & =0 & & \text { in }(0, \infty) \times \Omega, \\
w & =\sum_{j=1}^{K} u_{j} M\left(v_{j}\right) & & \text { on } \quad(0, \infty) \times \partial \Omega, \\
w(0) & =w_{0} & \text { in } \Omega . \\
\left(u_{1}, \ldots, u_{K}\right) & =\bar{u}, & \\
\bar{u}^{\prime}-D_{K} \bar{u}+G_{K} G_{K}^{*} \Pi_{2} P_{N} P w+G_{K} G_{K}^{*} \Pi_{3} \bar{u} & =0, & & \\
\bar{u}(0) & =0, & &
\end{array}\right.
$$

is exponentially stable, more precisely:

Proposition 35. Let $s \in[0,1]$ and $w_{0} \in \mathbf{V}_{0}^{s}(\Omega)$. Then (136) admits a unique solution

$$
(w, p, \bar{u}) \in W\left(\mathbf{V}^{s+1}(\Omega), \mathbf{V}_{0}^{s-1}(\Omega)\right) \times H^{-\frac{1}{2}+\frac{s}{2}}\left(H_{\sharp}^{s}(\Omega)\right) \times\left(H^{1}(\mathbb{R})\right)^{K}
$$

which obeys:

$$
\|w(t)\|_{\mathbf{V}^{s}(\Omega)}+\|\bar{u}(t)\|_{\mathbb{R}^{K}} \leq C\left\|w_{0}\right\|_{\mathbf{V}_{0}^{s}(\Omega)} e^{-\sigma t} \quad \text { for all } t \geq 0
$$


Proof. Let us introduce the operator $\mathcal{M}: \mathbb{R}^{K} \rightarrow \mathbf{V}^{2}(\Omega)$ defined by

$$
\mathcal{M}(\bar{u})=\sum_{j=1}^{K} u_{j}(I-P) D M\left(v_{j}\right) .
$$

By Proposition 24 it suffices to prove that the system

$$
\begin{aligned}
{ }^{t}(P w, \bar{u}) & =Y, \\
Y^{\prime}+\mathbb{A}_{\Pi} Y & =0, \quad Y(0)={ }^{t}\left(P w_{0}, 0\right), \\
(I-P) w & =\mathcal{M}(\bar{u})
\end{aligned}
$$

admits a unique solution $(w, \bar{u}) \in W\left(\mathbf{V}^{1+s}(\Omega), \mathbf{V}_{0}^{s-1}(\Omega)\right) \times\left(H^{1}(\mathbb{R})\right)^{K}$ which obeys (137). Moreover, according to (134) for $w_{0} \in \mathbf{V}_{0}^{s}(\Omega)$ we have ${ }^{t}\left(P w_{0}, 0\right)={ }^{t}\left(w_{0}, 0\right) \in \mathcal{D}\left(\mathbb{A}_{\Pi}^{\frac{s}{2}}\right)$ and, according to Proposition 17 with maximal regularity result for analytic semigroup (8), the corresponding solution $Y={ }^{t}(y, \bar{u})$ belongs to $W\left(\mathcal{D}\left(\mathbb{A}_{\Pi}^{\frac{1}{2}+\frac{s}{2}}\right),\left[\mathcal{D}\left(\mathbb{A}_{\Pi}^{* \frac{s}{2}-\frac{1}{2}}\right)\right]^{\prime}\right) \hookrightarrow W\left(\mathbf{V}_{n}^{s+1}(\Omega) \times \mathbb{R}^{K}, \mathbf{V}_{0}^{s-1}(\Omega) \times \mathbb{R}^{K}\right)$. Then we deduce that $\bar{u} \in\left(H^{1}(\mathbb{R})\right)^{K}$, $\mathcal{M}(\bar{u}) \in H^{1}\left(\mathbf{V}^{2}(\Omega)\right)$ and $w=y+\mathcal{M}(\bar{u}) \in W\left(\mathbf{V}^{s+1}(\Omega), \mathbf{V}_{0}^{s-1}(\Omega)\right)$ which allows to conclude. Finally, (137) is a consequence of (88).

Using Theorem 18 the solution of the system:

$$
\left\{\begin{array}{rlrl}
\frac{\partial w}{\partial t}-\nu \Delta w+\left(w_{S} \cdot \nabla\right) w+(w \cdot \nabla) w_{S}+(w \cdot \nabla) w+\nabla p & =0 & & \text { in } \quad(0, \infty) \times \Omega, \\
\nabla \cdot w & =0 & & \text { in }(0, \infty) \times \Omega, \\
w & =\sum_{j=1}^{K} u_{j} M\left(v_{j}\right) & & \text { on } \quad(0, \infty) \times \partial \Omega, \\
w(0) & =w_{0} & & \text { in } \quad \Omega, \\
\left(u_{1}, \ldots, u_{K}\right) & =\bar{u}, \\
\bar{u}^{\prime}-D_{K} \bar{u}+G_{K} G_{K}^{*} \Pi_{2} P_{N} P w+G_{K} G_{K}^{*} \Pi_{3} \bar{u} & =0, & & \\
\bar{u}(0) & =0, & &
\end{array}\right.
$$

is exponentially stable, more precisely:

Theorem 36. Let $s \in\left[\frac{d-2}{2}, 1\right]$ and $w_{0} \in \mathbf{V}_{0}^{s}(\Omega)$. There exists $\mu>0$ such that if $\left\|w_{0}\right\|_{\mathbf{V}_{0}^{s}(\Omega)} \leq \mu$ then (139) admits a solution $(w, p, \bar{u}) \in W\left(\mathbf{V}^{s+1}(\Omega), \mathbf{V}_{0}^{s-1}(\Omega)\right) \times H^{-\frac{1}{2}+\frac{s}{2}}\left(H_{\sharp}^{s}(\Omega)\right) \times\left(H^{1}(\mathbb{R})\right)^{K}$ which is unique within the class of function of $\left.\left(L_{\text {loc }}^{2}\left(\mathbf{V}^{s+1} \Omega\right)\right) \cap L_{\text {loc }}^{\infty}\left(\mathbf{V}^{s}(\Omega)\right)\right) \times H_{\text {loc }}^{-\frac{1}{2}+\frac{s}{2}}\left(H_{\sharp}^{s}(\Omega)\right) \times\left(H_{\text {loc }}^{1}(\mathbb{R})\right)^{K}$ and which obeys:

$$
\|w(t)\|_{\mathbf{V}^{s}(\Omega)}+\|\bar{u}(t)\|_{\mathbb{R}^{K}} \leq C\left\|w_{0}\right\|_{\mathbf{V}_{0}^{s}(\Omega)} e^{-\sigma t} \quad \text { for all } t \geq 0 .
$$

Proof. With $\mathcal{M}: \mathbb{R}^{K} \rightarrow \mathbf{V}^{2}(\Omega)$ given by (138) we set:

$$
N\left(\begin{array}{c}
y \\
\bar{u}
\end{array}\right) \stackrel{\text { def }}{=}\left(\begin{array}{c}
P((y+\mathcal{M}(\bar{u})) \cdot \nabla)(y+\mathcal{M}(\bar{u})) \\
0
\end{array}\right),
$$

and by Proposition 24 it suffices to prove that for some $\mu>0$ and $\left\|w_{0}\right\|_{\mathbf{V}_{0}^{s}(\Omega)} \leq \mu$ the system

$$
\begin{aligned}
{ }^{t}(P w, \bar{u}) & =Y \\
Y^{\prime}+\mathbb{A}_{\Pi} Y & =N(Y), \quad Y(0)={ }^{t}\left(P w_{0}, 0\right), \\
(I-P) w & =\mathcal{M}(\bar{u})
\end{aligned}
$$

admits a solution $(w, \bar{u}) \in W\left(\mathbf{V}^{1+s}(\Omega), \mathbf{V}_{0}^{s-1}(\Omega)\right) \times\left(H^{1}(\mathbb{R})\right)^{K}$, unique within the class of functions which belong to $\left(L_{\text {loc }}^{2}\left(\mathbf{V}^{s+1}(\Omega)\right) \cap L_{\text {loc }}^{\infty}\left(\mathbf{V}^{s}(\Omega)\right)\right) \times\left(H_{\text {loc }}^{1}(\mathbb{R})\right)^{K}$, and that this solution obeys (140). Then since $N$ obeys (92), which is an easy consequence of (102), (103) and of (134),(135), it suffices to apply Theorem 18.

Remark 37. If we choose $D_{K}=0$ and $G_{K}$ equal to the identity in $\mathbb{R}^{K}$ (see Remark 9) then the ordinary differential equation satisfied by $\bar{u}$ has the simpler form:

$$
\bar{u}^{\prime}+\Pi_{3} \bar{u}=-\Pi_{2} P_{N} P w .
$$




\section{References}

[1] M. Badra. Local stabilization of the Navier-Stokes equations with a feedback controller localized in an open subset of the domain. Numer. Funct. Anal. Optim., 28(5-6):559-589, 2007.

[2] M. Badra. Feedback stabilization of the 2-D and 3-D navier-stokes equations based on an extended system. ESAIM: Control, Optimisation and Calculus of Variations, 15(4):934-968, 2009.

[3] M. Badra. Lyapunov function and local feedback boundary stabilization of the Navier-Stokes equations. SIAM J. Control Optim., 48(3):1797-1830, 2009.

[4] V. Barbu. Stabilization of Navier-Stokes Flow. Communications and Control Engineering. Springer Verlag, Berlin-London, 2010.

[5] V. Barbu, I. Lasiecka, and R. Triggiani. Abstract settings for tangential boundary stabilization of NavierStokes equations by high- and low-gain feedback controllers. Nonlinear Anal., 64(12):2704-2746, 2006.

[6] V. Barbu, I. Lasiecka, and R. Triggiani. Tangential boundary stabilization of Navier-Stokes equations. Mem. Amer. Math. Soc., 181(852):x+128, 2006.

[7] V. Barbu, I. Lasiecka, and R. Triggiani. Local exponential stabilization strategies of the Navier-Stokes equations, $d=2,3$, via feedback stabilization of its linearization. In Control of coupled partial differential equations, volume 155 of Internat. Ser. Numer. Math., pages 13-46. Birkhäuser, Basel, 2007.

[8] V. Barbu and R. Triggiani. Internal stabilization of Navier-Stokes equations with finite-dimensional controllers. Indiana Univ. Math. J., 53(5):1443-1494, 2004.

[9] A. Bensoussan, G. Da Prato, M. C. Delfour, and S. K. Mitter. Representation and control of infinite dimensional systems. Systems \& Control: Foundations \& ApplicationsBirkhäuser Boston Inc., Boston, MA, second edition, 2007.

[10] F. Boyer and P. Fabrie. Éléments d'analyse pour l'étude de quelques modèles d'écoulements de fluides visqueux incompressibles, volume 52 of Mathématiques $\&$ Applications (Berlin) [Mathematics $\&$ Applications]. Springer-Verlag, Berlin, 2006.

[11] J.-M. Buchot and J.-P. Raymond. A linearized model for boundary layer equations. In Optimal control of complex structures (Oberwolfach, 2000), volume 139 of Internat. Ser. Numer. Math., pages 31-42. Birkhäuser, Basel, 2002.

[12] J.-M. Buchot and J.-P. Raymond. The linearized Crocco equation. J. Math. Fluid Mech., 8(4):510-541, 2006.

[13] J.-M. Buchot and J.-P. Raymond. Feedback stabilization of a boundary layer equation, Part 2: Nonhomogeneous state equations and numerical simulations. to appear in Applied Math. Res. Express, 2009.

[14] J.-M. Buchot and J.-P. Raymond. Feedback stabilization of a boundary layer equation, Part 1: Homogeneous state equations. to appear in ESAIM COCV, 2009.

[15] P. Constantin and C. Foias. Navier-Stokes equations. Chicago Lectures in Mathematics. University of Chicago Press, Chicago, IL, 1988.

[16] J.-M. Coron. Control and nonlinearity, volume 136 of Mathematical Surveys and Monographs. American Mathematical Society, Providence, RI, 2007.

[17] R. Denk, M. Hieber, and J. Prüss. R-boundedness, Fourier multipliers and problems of elliptic and parabolic type. Mem. Amer. Math. Soc., 166(788):viii+114, 2003.

[18] E. Fernández-Cara, S. Guerrero, O. Yu. Imanuvilov, and J.-P. Puel. Local exact controllability of the Navier-Stokes system. J. Math. Pures Appl. (9), 83(12):1501-1542, 2004.

[19] H. Fujita and H. Morimoto. On fractional powers of the Stokes operator. Proc. Japan Acad., 46:1141-1143, 1970. 
[20] A. V. Fursikov. Stabilizability of two-dimensional Navier-Stokes equations with help of a boundary feedback control. J. Math. Fluid Mech., 3(3):259-301, 2001.

[21] G. P. Galdi. An introduction to the mathematical theory of the Navier-Stokes equations. Vol. I. Linearized steady problems, volume 38 of Springer Tracts in Natural Philosophy. Springer-Verlag, New York, 1994.

[22] G. P. Galdi. An introduction to the mathematical theory of the Navier-Stokes equations. Vol. II, Nonlinear steady problems, volume 39 of Springer Tracts in Natural Philosophy. Springer-Verlag, New York, 1994.

[23] V. Girault and P.-A. Raviart. Finite element methods for Navier-Stokes equations. Theory and algorithms, volume 5 of Springer Series in Computational Mathematics. Springer-Verlag, Berlin, 1986.

[24] P. Grisvard. Caractérisation de quelques espaces d'interpolation. Arch. Rational Mech. Anal., 25:40-63, 1967.

[25] S. Guerrero. Local exact controllability to the trajectories of the Boussinesq system. Ann. Inst. H. Poincaré Anal. Non Linéaire, 23(1):29-61, 2006.

[26] S. Guerrero. Controllability of systems of Stokes equations with one control force: existence of insensitizing controls. Ann. Inst. H. Poincaré Anal. Non Linéaire, 24(6):1029-1054, 2007.

[27] E. Hille and R. S. Phillips. Functional analysis and semi-groups. American Mathematical Society Colloquium Publications, vol. 31. American Mathematical Society, Providence, R. I., 1957. rev. ed.

[28] O. Yu. Imanuvilov. Remarks on exact controllability for the Navier-Stokes equations. ESAIM Control Optim. Calc. Var., 6:39-72 (electronic), 2001.

[29] T. Kato. Perturbation theory for linear operators. Classics in Mathematics. Springer-Verlag, Berlin, 1995. Reprint of the 1980 edition.

[30] C. Lefter. Feedback stabilization of 2D Navier-Stokes equations with Navier slip boundary conditions. Nonlinear Anal., 70(1):553-562, 2009.

[31] A. Pazy. Semigroups of linear operators and applications to partial differential equations, volume 44 of Applied Mathematical Sciences. Springer-Verlag, New York, 1983.

[32] J.-P. Raymond. Feedback boundary stabilization of the two-dimensional Navier-Stokes equations. SIAM J. Control Optim., 45(3):790-828 (electronic), 2006.

[33] J.-P. Raymond. Feedback boundary stabilization of the three-dimensional incompressible Navier-Stokes equations. J. Math. Pures Appl. (9), 87(6):627-669, 2007.

[34] J.-P. Raymond. Stokes and Navier-Stokes equations with nonhomogeneous boundary conditions. Ann. Inst. H. Poincaré Anal. Non Linéaire, 24(6):921-951, 2007.

[35] J-P. Raymond and L. Thevenet. Boundary feedback stabilization of the two dimensional Navier-Stokes equations with finite dimensional controllers. Issue in Discrete and Continuous Dynamical Systems A, 27(3):1159-1187, 2010.

[36] D. L. Russell. Controllability and stabilizability theory for linear partial differential equations: recent progress and open questions. SIAM Rev., 20(4):639-739, 1978.

[37] T. Takahashi. Analysis of strong solutions for the equations modeling the motion of a rigid-fluid system in a bounded domain. Adv. Differential Equations, 8(12):1499-1532, 2003.

[38] H. Triebel. Interpolation theory, function spaces, differential operators. Johann Ambrosius Barth, Heidelberg, second edition, 1995.

[39] M. Tucsnak and G. Weiss. Observation and control for operator semigroups. Birkhäuser Advanced Texts: Basler Lehrbücher. [Birkhäuser Advanced Texts: Basel Textbooks]. Birkhäuser Verlag, Basel, 2009.

[40] A. Yagi. Coïncidence entre des espaces d'interpolation et des domaines de puissances fractionnaires d'opérateurs. C. R. Acad. Sci. Paris Sér. I Math., 299(6):173-176, 1984.

[41] J. Zabczyk. Mathematical control theory. Modern Birkhäuser Classics. Birkhäuser Boston Inc., Boston, MA, 2008. An introduction, Reprint of the 1995 edition. 